\title{
On kernel-based estimation of conditional Kendall's tau: finite-distance bounds and asymptotic behavior
}

https://doi.org/10.1515/demo-2019-0016

Received May 29, 2019; accepted August 31, 2019

\begin{abstract}
We study nonparametric estimators of conditional Kendall's tau, a measure of concordance between two random variables given some covariates. We prove non-asymptotic pointwise and uniform bounds, that hold with high probabilities. We provide "direct proofs" of the consistency and the asymptotic law of conditional Kendall's tau. A simulation study evaluates the numerical performance of such nonparametric estimators. An application to the dependence between energy consumption and temperature conditionally to calendar days is finally provided.
\end{abstract}

Keywords: conditional dependence measures, kernel smoothing, conditional Kendall's tau

MSC: $62 \mathrm{H} 20,62 \mathrm{G} 05,62 \mathrm{G} 08,62 \mathrm{G} 20$

\section{Introduction}

In the field of dependence modeling, it is common to work with dependence measures. Contrary to usual linear correlations, most of them have the advantage of being defined without any condition on moments, and of being invariant to changes in the underlying marginal distributions. Such summaries of information are very popular and can be explicitly written as functionals of the underlying copulas: Kendall's tau, Spearman's rho, Blomqvist's coefficient... See Nelsen [30] for an introduction. In particular, for more than a century (Spearman (1904), Kendall (1938)), Kendall's tau has become a popular dependence measure in [-1, 1]. It quantifies the positive or negative dependence between two random variables $X_{1}$ and $X_{2}$. Denoting by $C_{1,2}$ the unique underlying copula of $\left(X_{1}, X_{2}\right)$ that is assumed to be continuous, their Kendall's tau can be directly defined as

$$
\begin{aligned}
\tau_{1,2} & :=4 \int_{[0,1]^{2}} C_{1,2}\left(u_{1}, u_{2}\right) C_{1,2}\left(d u_{1}, d u_{2}\right)-1 \\
& =\mathbb{P}\left(\left(X_{1,1}-X_{2,1}\right)\left(X_{1,2}-X_{2,2}\right)>0\right)-\mathbb{P}\left(\left(X_{1,1}-X_{2,1}\right)\left(X_{1,2}-X_{2,2}\right)<0\right),
\end{aligned}
$$

where $\left(X_{i, 1}, X_{i, 2}\right)_{i=1,2}$ are two independent versions of $\mathbf{X}:=\left(X_{1}, X_{2}\right)$. This measure is then interpreted as the probability of observing a concordant pair minus the probability of observing a discordant pair. See [22] for an historical perspective on Kendall's tau. Its inference is discussed in many textbooks (see [18] or [24], e.g.). Its links with copulas and other dependence measures can be found in [30] or [20].

Similar dependence measures can be introduced in a conditional setup, when a $p$-dimensional covariate $\mathbf{Z}$ is available. When hundreds of papers refer to Kendall's tau, only a few of them have considered conditional Kendall's tau (as defined below) until now. The goal is now to model the dependence between the two

\footnotetext{
*Corresponding Author: Alexis Derumigny: CREST-ENSAE and University of Twente, 5 Drienerlolaan, 7522 NB Enschede, Netherlands, E-mail: a.f.f.derumigny@utwente.nl.

Jean-David Fermanian: CREST-ENSAE, 5, avenue Henry Le Chatelier, 91764 Palaiseau cedex, France,

E-mail: jean-david.fermanian@ensae.fr.
} 
components $X_{1}$ and $X_{2}$, given the vector of covariates $\mathbf{Z}$. Logically, we can invoke the conditional copula ${ }^{1}$ $C_{1,2 \mid \mathbf{Z}=\mathbf{z}}$ of $\left(X_{1}, X_{2}\right)$ given $\mathbf{Z}=\mathbf{z}$ for any point $\mathbf{z} \in \mathbb{R}^{p}$, and the corresponding conditional Kendall's tau would be simply defined as

$$
\begin{aligned}
\tau_{1,2 \mid \mathbf{Z}=\mathbf{z}} & :=4 \int_{[0,1]^{2}} C_{1,2 \mid \mathbf{Z}=\mathbf{z}}\left(u_{1}, u_{2}\right) C_{1,2 \mid \mathbf{Z}=\mathbf{z}}\left(d u_{1}, d u_{2}\right)-1 \\
& =\mathbb{P}\left(\left(X_{1,1}-X_{2,1}\right)\left(X_{1,2}-X_{2,2}\right)>0 \mid \mathbf{Z}_{1}=\mathbf{Z}_{2}=\mathbf{z}\right)-\mathbb{P}\left(\left(X_{1,1}-X_{2,1}\right)\left(X_{1,2}-X_{2,2}\right)<0 \mid \mathbf{Z}_{1}=\mathbf{Z}_{2}=\mathbf{z}\right),
\end{aligned}
$$

where $\left(X_{i, 1}, X_{i, 2}, \mathbf{Z}_{i}\right)_{i=1,2}$ are two independent versions of $\left(X_{1}, X_{2}, \mathbf{Z}\right)$. As above, this is the probability of observing a concordant pair minus the probability of observing a discordant pair, conditionally on $\mathbf{Z}_{1}$ and $\mathbf{Z}_{2}$ being both equal to $\mathbf{z}$. Note that, as conditional copulas themselves, conditional Kendall's taus are invariant w.r.t. increasing transformations of the conditional margins $X_{1}$ and $X_{2}$, given $\mathbf{Z}$. Of course, if $\mathbf{Z}$ is independent of $\left(X_{1}, X_{2}\right)$ then, for every $\mathbf{z} \in \mathbb{R}^{p}$, the conditional Kendall's tau $\tau_{1,2 \mid \mathbf{Z}=\mathbf{z}}$ is equal to the (unconditional) Kendall's tau $\tau_{1,2}$.

Conditional Kendall's tau, and more generally conditional dependence measures, are of interest per se because they allow to summarize the evolution of the dependence between $X_{1}$ and $X_{2}$, when the covariate $\mathbf{Z}$ is changing. Surprisingly, their nonparametric estimates have been introduced in the literature only a few years ago ([15],[40],[13]) and their properties have not yet been fully studied in depth. Indeed, until now and to the best of our knowledge, the theoretical properties of nonparametric conditional Kendall's tau estimates have been obtained "in passing" in the literature, as a sub-product of the weak-convergence of conditional copula processes ([40]) or as intermediate quantities that will be "plugged-in" ([12]). Therefore, such properties have been stated under too demanding assumptions. In particular, some assumptions were related to the estimation of conditional margins, while this is not required because Kendall's tau are based on ranks. In this paper, we directly study nonparametric estimates $\hat{\tau}_{1,2 \mid \mathbf{z}}$ without relying on the theory/inference of copulas. Therefore, we will state their main usual statistical properties: exponential bounds in probability, consistency, asymptotic normality.

Our $\tau_{1,2 \mid \mathbf{Z}=\mathbf{z}}$ has not to be confused with the so-called "conditional Kendall's tau" in the case of truncated data ([39], [28]), in the case of semi-competing risk models ([23], [19]), or for other partial information schemes ([6], [21], among others). Indeed, particularly in biostatistics or reliability, the inference of dependence models under truncation/censoring can be led by considering some types of conditional Kendall's tau, given some algebraic relationships among the underlying random variables. This would induce conditioning by subsets. At the opposite, we will consider only pointwise conditioning events in this paper, under a nonparametric point-of-view. Nonetheless, such pointwise events can be found in the literature, but in some parametric or semi-parametric particular frameworks, as for the identifiability of frailty distributions in bivariate proportional models ([31], [27]). Other related papers are [3] or [25], that are dealing with extreme co-movements (bivariate extreme-value theory). There, the tail conditioning events of Kendall's tau have probabilities that go to zero with the sample size.

In Section 2, different kernel-based estimators of the conditional Kendall's tau are discussed. Moreover, we propose a cross-validation criterion to select the associated bandwidth. In Section 3, numerous original theoretical properties of the latter estimators are proved: at first, finite distance exponential bounds in probability (pointwise and uniformly w.r.t. z); then, under an asymptotic point-of-view, pointwise and uniform consistency; and finally the asymptotic normality of conditional Kendall's tau under unrestrictive assumptions (see below) and with an explicit limiting law. A short simulation study is provided in Section 4. Proofs are postponed into the appendix.

1 The conditional copula of $X_{1}$ and $X_{2}$ given $\mathbf{Z}=\mathbf{z}$ can be defined almost surely as the unique copula of the conditional c.d.f. $F_{X_{1}, X_{2} \mid \mathbf{Z}=\mathbf{z}}$ by Sklar's theorem. It was introduced by Patton [33, 34]. 


\section{Definition of several kernel-based estimators of $\tau_{1,2 \mid \mathrm{z}}$}

Let $\left(X_{i, 1}, X_{i, 2}, \mathbf{Z}_{i}\right), i=1, \ldots, n$ be an i.i.d. sample distributed as $\left(X_{1}, X_{2}, \mathbf{Z}\right)$, and $n \geq 2$. Assuming continuous underlying distributions, there are several equivalent ways of defining the conditional Kendall's tau:

$$
\begin{aligned}
\tau_{1,2 \mid \mathbf{Z}=\mathbf{z}} & =4 \mathbb{P}\left(X_{1,1}>X_{2,1}, X_{1,2}>X_{2,2} \mid \mathbf{Z}_{1}=\mathbf{Z}_{2}=\mathbf{z}\right)-1 \\
& =1-4 \mathbb{P}\left(X_{1,1}>X_{2,1}, X_{1,2}<X_{2,2} \mid \mathbf{Z}_{1}=\mathbf{Z}_{2}=\mathbf{z}\right) \\
& =\mathbb{P}\left(\left(X_{1,1}-X_{2,1}\right)\left(X_{1,2}-X_{2,2}\right)>0 \mid \mathbf{Z}_{1}=\mathbf{Z}_{2}=\mathbf{z}\right)-\mathbb{P}\left(\left(X_{1,1}-X_{2,1}\right)\left(X_{1,2}-X_{2,2}\right)<0 \mid \mathbf{Z}_{1}=\mathbf{Z}_{2}=\mathbf{z}\right) .
\end{aligned}
$$

Motivated by each of the latter expressions, we introduce several kernel-based estimators of $\tau_{1,2 \mid \mathbf{Z}=\mathbf{z}}$ :

$$
\begin{aligned}
& \hat{\tau}_{1,2 \mid \mathbf{Z}=\mathbf{z}}^{(1)}:=4 \sum_{i=1}^{n} \sum_{j=1}^{n} w_{i, n}(\mathbf{z}) w_{j, n}(\mathbf{z}) \mathbb{1}\left\{X_{i, 1}<X_{j, 1}, X_{i, 2}<X_{j, 2}\right\}-1, \\
& \hat{\tau}_{1,2 \mid \mathbf{Z}=\mathbf{z}}^{(2)}:=\sum_{i=1}^{n} \sum_{j=1}^{n} w_{i, n}(\mathbf{z}) w_{j, n}(\mathbf{z})\left(\mathbb{1}\left\{\left(X_{i, 1}-X_{j, 1}\right)\left(X_{i, 2}-X_{j, 2}\right)>0\right\}-\mathbb{1}\left\{\left(X_{i, 1}-X_{j, 1}\right)\left(X_{i, 2}-X_{j, 2}\right)<0\right\}\right), \\
& \hat{\tau}_{1,2 \mid \mathbf{Z}=\mathbf{z}}^{(3)}:=1-4 \sum_{i=1}^{n} \sum_{j=1}^{n} w_{i, n}(\mathbf{z}) w_{j, n}(\mathbf{z}) \mathbb{1}\left\{X_{i, 1}<X_{j, 1}, X_{i, 2}>X_{j, 2}\right\},
\end{aligned}
$$

where $\mathbb{1}$ denotes the indicator function, $w_{i, n}$ is a sequence of weights given by

$$
w_{i, n}(\mathbf{z})=\frac{K_{h}\left(\mathbf{Z}_{i}-\mathbf{z}\right)}{\sum_{j=1}^{n} K_{h}\left(\mathbf{Z}_{j}-\mathbf{z}\right)},
$$

with $K_{h}(\cdot):=h^{-p} K(\cdot / h)$ for some kernel $K$ on $\mathbb{R}^{p}$, and $h=h(n)$ denotes a usual bandwidth sequence that tends to zero when $n \rightarrow \infty$. In this paper, we have chosen usual Nadaraya-Watson weights. Obviously, there are alternatives (local linear, Priestley-Chao, Gasser-Müller, etc., weight), that would lead to different theoretical results.

The estimators $\hat{\tau}_{1,2 \mid \mathbf{Z}=\mathbf{z}}^{(1)}, \hat{\tau}_{1,2 \mid \mathbf{Z}=\mathbf{z}}^{(2)}$ and $\hat{\tau}_{1,2 \mid \mathbf{Z}=\mathbf{z}}^{(3)}$ look similar, but they are nevertheless different, as shown in Proposition 1. These differences are due to the fact that all the $\hat{\tau}_{1,2 \mid \mathbf{Z}=\mathbf{z}}^{(k)}, k=1,2,3$ are affine transformations of a double-indexed sum, on every pair $(i, j)$, including the diagonal terms where $i=j$. The treatment of these diagonal terms is different for each of the three estimators defined above. Indeed, setting $s_{n}:=\sum_{i=1}^{n} w_{i, n}^{2}(\mathbf{z})$, it can be easily proved that $\hat{\tau}_{1,2 \mid \mathbf{Z}=\mathbf{z}}^{(1)}$ takes values in the interval $\left[-1,1-2 s_{n}\right], \hat{\tau}_{1,2 \mid \mathbf{Z}=\mathbf{z}}^{(2)}$ in $\left[-1+s_{n}, 1-s_{n}\right]$, and $\hat{\tau}_{1,2 \mid \mathbf{Z}=\mathbf{z}}^{(3)}$ in $\left[-1+2 s_{n}, 1\right]$. Moreover, there exists a direct relationship between these estimators, given by the following proposition.

Proposition 1. Almost surely, $\hat{\tau}_{1,2 \mid \mathbf{Z}=\mathbf{z}}^{(1)}+s_{n}=\hat{\tau}_{1,2 \mid \mathbf{Z}=\mathbf{z}}^{(2)}=\hat{\tau}_{1,2 \mid \mathbf{Z}=\mathbf{z}}^{(3)}-s_{n}$, where $s_{n}:=\sum_{i=1}^{n} w_{i, n}^{2}(\mathbf{z})$.

This proposition is proved in A.2. As a consequence, we can easily rescale the previous estimators so that the new estimator will take values in the whole interval $[-1,1]$. This would yield

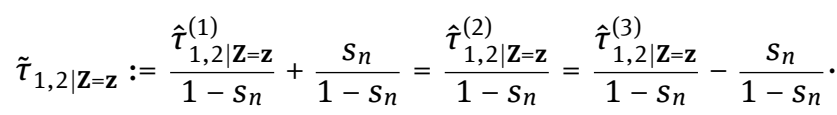

Note that none of the latter estimators depends on any estimation of conditional marginal distributions. In other words, we only have to conveniently choose the weights $w_{i, n}$ to obtain an estimator of the conditional Kendall's tau. This is coherent with the fact that conditional Kendall's taus are invariant with respect to conditional marginal distributions. Moreover, note that, in the definition of our estimators, the inequalities are strict (there are no terms corresponding to the cases $i=j$ ). This is inline with the definition of (conditional) Kendall's tau itself through concordant/discordant pairs of observations. 
The definition of $\hat{\tau}_{1,2 \mid \mathbf{Z}=\mathbf{z}}^{(1)}$ can be motivated as follows. For $j=1$, 2, let $\hat{F}_{j \mid \mathbf{Z}}(\cdot \mid \mathbf{Z}=\mathbf{z})$ be an estimator of the conditional cdf of $X_{j}$ given $\mathbf{Z}=\mathbf{z}$. Then, a usual estimator of the conditional copula of $X_{1}$ and $X_{2}$ given $\mathbf{Z}=\mathbf{z}$ is

$$
\hat{C}_{1,2 \mid \mathbf{Z}}\left(u_{1}, u_{2} \mid \mathbf{Z}=\mathbf{z}\right):=\sum_{i=1}^{n} w_{i, n}(\mathbf{z}) \mathbb{1}\left\{\hat{F}_{1 \mid \mathbf{Z}}\left(X_{i, 1} \mid \mathbf{Z}=\mathbf{z}\right) \leq u_{1}, \hat{F}_{2 \mid \mathbf{Z}}\left(X_{i, 2} \mid \mathbf{Z}=\mathbf{z}\right) \leq u_{2}\right\} .
$$

See [40] or [13], e.g. The latter estimator of the conditional copula can be plugged into (1) to define an estimator of the conditional Kendall's tau itself:

$$
\begin{aligned}
\hat{\tau}_{1,2 \mid \mathbf{Z}=\mathbf{z}} & :=4 \int \hat{C}_{1,2 \mid \mathbf{Z}}\left(u_{1}, u_{2} \mid \mathbf{Z}=\mathbf{z}\right) \hat{C}_{1,2 \mid \mathbf{Z}}\left(d u_{1}, d u_{2} \mid \mathbf{Z}=\mathbf{z}\right)-1 \\
& =4 \sum_{j=1}^{n} w_{j, n}(\mathbf{z}) \hat{C}_{1,2 \mid \mathbf{Z}}\left(\hat{F}_{1 \mid \mathbf{Z}}\left(X_{j, 1} \mid \mathbf{Z}=\mathbf{z}\right), \hat{F}_{2 \mid \mathbf{Z}}\left(X_{j, 2} \mid \mathbf{Z}=\mathbf{z}\right) \mid \mathbf{Z}=\mathbf{z}\right)-1 .
\end{aligned}
$$

Since the functions $\hat{F}_{j \mid \mathbf{Z}}(\cdot \mid \mathbf{Z}=\mathbf{z})$ are non-decreasing, this reduces to

$$
\begin{aligned}
\hat{\tau}_{1,2 \mid \mathbf{Z}=\mathbf{z}}= & 4 \sum_{i=1}^{n} \sum_{j=1}^{n} w_{i, n}(\mathbf{z}) w_{j, n}(\mathbf{z}) \mathbb{1}\left\{X_{i, 1} \leq X_{j, 1}, X_{i, 2} \leq X_{j, 2}\right\}-1 \\
= & 4 \sum_{i=1}^{n} \sum_{j=1}^{n} w_{i, n}(\mathbf{z}) w_{j, n}(\mathbf{z}) \mathbb{1}\left\{X_{i, 1}<X_{j, 1}, X_{i, 2}<X_{j, 2}\right\}-1+o_{P}(1)=\hat{\tau}_{1,2 \mid \mathbf{Z}=\mathbf{z}}^{(1)}+o_{P}(1) .
\end{aligned}
$$

Veraverbeke et al. [40], Subsection 3.2, introduced their estimator of $\tau_{1,2 \mid \mathbf{Z}=\mathbf{z}}$ by (3) for a univariate conditioning variable. Note that this estimator is the same as the one studied in $[15, p .4]$, i.e. $\hat{\tau}_{1,2 \mid \mathbf{Z}=\mathbf{z}}$. By the functional Delta-Method, they deduced its asymptotic normality as a sub-product of the weak convergence of the process $\left.\sqrt{n h}\left(\hat{C}_{1,2 \mid \mathbf{Z}} \mathbf{Z} \cdot, \cdot \mid z\right)-C_{1,2 \mid \mathbf{Z}}(\cdot, \cdot \mid z)\right)$ when $\mathbf{Z}$ is univariate. In our case, we will obtain more and stronger theoretical properties of $\hat{\tau}_{1,2 \mid \mathbf{Z}=\mathbf{z}}^{(1)}$ under weaker conditions by a more direct analysis based on ranks. In particular, we will not require any regularity condition on the conditional marginal distributions, contrary to [40]. Indeed, in the latter paper, it is required that $F_{j \mid \mathbf{Z}}(\cdot \mid \mathbf{Z}=\mathbf{z})$ has to be two times continuously differentiable (assumption $(\tilde{R} 3))$ and its inverse has to be continuous (assumption $(R 1))$. This is not satisfied for some simple univariate cdf as $F_{j}(t)=t \mathbb{1}(t \in[0,1]) / 2+\mathbb{1}(t \in(1,2]) / 2+t \mathbb{1}(t \in(2,4]) / 4+\mathbb{1}(t>4)$, for instance. Note that we could justify $\hat{\tau}_{1,2 \mid \mathbf{Z}=\mathbf{z}}^{(3)}$ in a similar way by considering conditional survival copulas.

Let us define $g_{1}, g_{2}, g_{3}$ by

$$
\begin{aligned}
& g_{1}\left(\mathbf{X}_{i}, \mathbf{X}_{j}\right):=4 \mathbb{1}\left\{X_{i, 1}<X_{j, 1}, X_{i, 2}<X_{j, 2}\right\}-1, \\
& g_{2}\left(\mathbf{X}_{i}, \mathbf{X}_{j}\right):=\mathbb{1}\left\{\left(X_{i, 1}-X_{j, 1}\right) \times\left(X_{i, 2}-X_{j, 2}\right)>0\right\}-\mathbb{1}\left\{\left(X_{i, 1}-X_{j, 1}\right) \times\left(X_{i, 2}-X_{j, 2}\right)<0\right\}, \\
& g_{3}\left(\mathbf{X}_{i}, \mathbf{X}_{j}\right):=1-4 \mathbb{1}\left\{X_{i, 1}<X_{j, 1}, X_{i, 2}>X_{j, 2}\right\},
\end{aligned}
$$

where, for $i=1, \ldots, n$, we set $\mathbf{X}_{i}:=\left(X_{i, 1}, X_{i, 2}\right)$. Clearly, $\hat{\tau}_{1,2 \mid \mathbf{z}}^{(k)}$ is a smoothed estimator of $\mathbb{E}\left[g_{k}\left(\mathbf{X}_{1}, \mathbf{X}_{2}\right) \mid \mathbf{Z}_{1}=\right.$ $\left.\mathbf{Z}_{2}=\mathbf{z}\right], k=1,2,3$.

Note that such dependence measures are of interest for the purpose of estimating (conditional or unconditional) copula models too. Indeed, several popular parametric families of copulas have a simple one-to-one mapping between their parameter and the associated Kendall's tau (or Spearman's rho): Gaussian, Student with a fixed degree of freedom, Clayton, Gumbel and Frank copulas, etc. Then, assume for instance that the conditional copula $C_{1,2 \mid \mathbf{Z}=\mathbf{z}}$ is a Gaussian copula with a parameter $\rho(\mathbf{z})$. Then, by estimating its conditional Kendall's tau $\tau_{1,2 \mid \mathbf{Z}=\mathbf{z}}$, we get an estimate of the corresponding parameter $\rho(\mathbf{z})$, and finally of the conditional copula itself. See [36], e.g.

The choice of the bandwidth $h$ could be done in a data-driven way, following the general conditional U-statistics framework detailed in Dony and Mason [10, Section 2]. Indeed, for any $k \in\{1,2,3\}$ and $\mathbf{z} \in \mathbb{R}^{p}$, denote by $\hat{\tau}_{-(i, j), 1,2 \mid \mathbf{Z}=\mathbf{z}}^{(h, k)}$ the estimator $\hat{\tau}_{1,2 \mid \mathbf{Z}=\mathbf{z}}^{(k)}$ that is made with the smoothing parameter $h$ and our dataset, when the $i$-th and $j$-th observations have been removed. As a consequence, the random function $\hat{\tau}_{-(i, j), 1,2 \mid \mathbf{Z}=\text {. }}^{(h, k)}$ 
is independent of $\left(\left(\mathbf{X}_{i}, \mathbf{Z}_{i}\right),\left(\mathbf{X}_{j}, \mathbf{Z}_{j}\right)\right)$. As usual with kernel methods, it would be tempting to propose $h$ as the minimizer of the cross-validation criterion

$$
C V_{D M}(h):=\frac{2}{n(n-1)} \sum_{i, j=1}^{n}\left(g_{k}\left(\mathbf{X}_{i}, \mathbf{X}_{j}\right)-\hat{\tau}_{-(i, j), 1,2 \mid \mathbf{Z}=\left(\mathbf{Z}_{i}+\mathbf{Z}_{j}\right) / 2}^{(h, k)}\right)^{2} K_{h}\left(\mathbf{Z}_{i}-\mathbf{Z}_{j}\right),
$$

for $k=1,2,3$ or for $\tilde{\tau}_{1,2 \mid \mathbf{Z}=.}$. The latter criterion would be a "naively localized" version of the usual crossvalidation method. Unfortunately, we observe that the function $h \mapsto C V_{D M}(h)$ is most often decreasing in the range of realistic bandwidth values. If we remove the weight $K_{h}\left(\mathbf{Z}_{i}-\mathbf{Z}_{j}\right)$, then there is no reason why $g_{k}\left(\mathbf{X}_{i}, \mathbf{X}_{j}\right)$ should be equal to $\hat{\tau}_{-(i, j), 1,2 \mid \mathbf{Z}=\left(\mathbf{Z}_{i}+\mathbf{Z}_{j}\right) / 2}^{(k)}$ (on average), and we are not interested in the prediction of concordance/discordance pairs for which the $Z_{i}$ and $Z_{j}$ are far apart. Therefore, a modification of this criteria is necessary. We propose to separate the choice of $h$ for the terms $g_{k}\left(\mathbf{X}_{i}, \mathbf{X}_{j}\right)-\hat{\tau}_{-(i, j), 1,2 \mid \mathbf{Z}=\left(\mathbf{Z}_{i}+\mathbf{Z}_{j}\right) / 2}^{(h, k)}$ and the selection of the "convenient pairs" of observations $(i, j)$. This leads to the new criterion

$$
C V_{\tilde{h}}(h):=\frac{2}{n(n-1)} \sum_{i, j=1}^{n}\left(g_{k}\left(\mathbf{X}_{i}, \mathbf{X}_{j}\right)-\hat{\tau}_{-(i, j), 1,2 \mid \mathbf{Z}=\left(\mathbf{Z}_{i}+\mathbf{Z}_{j}\right) / 2}^{(h, k)}\right)^{2} \tilde{K}_{\tilde{h}}\left(\mathbf{Z}_{i}-\mathbf{Z}_{j}\right),
$$

with a potentially different kernel $\tilde{K}$ and a new fixed tuning parameter $\tilde{h}$. Even if more complex procedures are possible, we suggest to simply choose $\tilde{K}(\mathbf{z}):=\mathbb{1}\left\{|\mathbf{z}|_{\infty} \leq 1\right\}$ and to calibrate $\tilde{h}$ so that only a fraction of the pairs $(i, j)$ has non-zero weights. In practice, set $\tilde{h}$ as the empirical quantile of $\left(\left\{\left|\mathbf{Z}_{i}-\mathbf{Z}_{j}\right|_{\infty}: 1 \leq i<j \neq n\right\}\right.$ of order $2 N_{\text {pairs }} /(n(n-1))$, where $N_{\text {pairs }}$ is the number of pairs we want to keep.

\section{Theoretical results}

\subsection{Finite distance bounds}

Hereafter, we will consider the behavior of conditional Kendall's tau estimates given $\mathbf{Z}=\mathbf{z}$ belongs to some fixed open and bounded subset $z$ in $\mathbb{R}^{p}$. For the moment, let us state an instrumental result that is of interest per se. Let $\hat{f}_{\mathbf{Z}}(\mathbf{z}):=n^{-1} \sum_{j=1}^{n} K_{h}\left(\mathbf{Z}_{j}-\mathbf{z}\right)$ be the usual kernel estimator of the density $f_{\mathbf{Z}}$ of the conditioning variable $\mathbf{Z}$. Note that the estimators $\hat{\tau}_{1,2 \mid \mathbf{Z}=\mathbf{z}}^{(k)}, k=1, \ldots, 3$ are well-behaved only whenever $\hat{f}_{\mathbf{Z}}(\mathbf{z})>0$. Denote the joint density of $(\mathbf{X}, \mathbf{Z})$ by $f_{\mathbf{X}, \mathbf{Z}}$. In our study, we need some usual conditions of regularity.

Assumption 3.1. (a) The kernel $K$ is bounded, and set $\|K\|_{\infty}=: C_{K}$. (b) It is symmetrical in the sense that $K(\mathbf{u})=K(-\mathbf{u})$ for every $\mathbf{u} \in \mathbb{R}^{p}$ and satisfies $\int K=1, \int|K|<\infty, \int K^{2}<\infty$. (c) This kernel is of order $\alpha$ for some integer $\alpha>1$ : for all $j=1, \ldots, \alpha-1$ and every indices $i_{1}, \ldots, i_{j}$ in $\{1, \ldots, p\}, \int K(\mathbf{u}) u_{i_{1}} \ldots u_{i_{j}} d \mathbf{u}=0 .(d)$ Moreover, $\mathbb{E}\left[K_{h}(\mathbf{Z}-\mathbf{z})\right]>0$ for every $\mathbf{z} \in Z$ and $h>0$. Set $\tilde{K}(\cdot):=K^{2}(\cdot) / \int K^{2}$ and $\|\tilde{K}\|_{\infty}=: C_{\tilde{K}}$.

Assumption 3.2. $f_{\mathrm{Z}}$ is $\alpha$-times continuously differentiable on $z^{2}$ and there exists a constant $C_{K, \alpha}>0$ s.t., for all $\mathbf{z} \in \mathcal{Z}$,

$$
\int|K|(\mathbf{u}) \sum_{i_{1}, \ldots, i_{\alpha}=1}^{p}\left|u_{i_{1}} \ldots u_{i_{\alpha}}\right| \sup _{t \in[0,1]}\left|\frac{\partial^{\alpha} f_{\mathbf{Z}}}{\partial z_{i_{1}} \ldots \partial z_{i_{\alpha}}}(\mathbf{z}+t h \mathbf{u})\right| d \mathbf{u} \leq C_{K, \alpha} .
$$

Moreover, $C_{\tilde{K}, 2}$ denotes a similar constant replacing $K$ by $\tilde{K}$ and $\alpha$ by two.

Assumption 3.3. There exist two positive constants $f_{\mathbf{Z}, \min }$ and $f_{\mathbf{Z}, \max }$ such that, for every $\mathbf{z} \in \mathcal{Z}, f_{\mathbf{Z}, \min } \leq f_{\mathbf{Z}}(\mathbf{z}) \leq$ $f_{\mathbf{Z}, \max }$.

2 This means that the partial derivatives $\partial^{k} f_{Z}(\mathbf{z}) / \partial z_{i_{1}} \cdots \partial z_{i_{k}}$ exist and are continuous for every $\mathbf{z} \in z$ and every $k$-uplet $\left(i_{1}, \ldots, i_{k}\right)$ of integers in $\{1, \ldots, p\}, k \leq \alpha$. 
Since $Z$ is bounded, Assumption 3.3 is most often satisfied with the commonly met continuous distribution.

Proposition 2. Under Assumptions 3.1-3.3 and if $C_{K, \alpha} h^{\alpha} / \alpha !<f_{\mathbf{Z}, \min }$, for any $\mathbf{z} \in Z$, the estimator $\hat{f}_{\mathbf{Z}}(\mathbf{z})$ is strictly positive with a probability larger than

$$
1-2 \exp \left(-n h^{p}\left(f_{\mathbf{Z}, \min }-C_{K, \alpha} h^{\alpha} / \alpha !\right)^{2} /\left(2 f_{\mathbf{Z}, \max } \int K^{2}+(2 / 3) C_{K}\left(f_{\mathbf{Z}, \min }-C_{K, \alpha} h^{\alpha} / \alpha !\right)\right)\right) .
$$

The latter proposition is proved in A.3. It guarantees that our estimators $\hat{\tau}_{1,2 \mid \mathbf{z}}^{(k)}, k=1, \ldots, 3$, are well-behaved with a probability close to one. The next regularity assumption is necessary to explicitly control the bias of $\hat{\tau}_{1,2 \mid \mathbf{Z}=\mathbf{z}}$.

Assumption 3.4. For every $\mathbf{x} \in \mathbb{R}^{2}, \mathbf{z} \mapsto f_{\mathbf{X}, \mathbf{Z}}(\mathbf{x}, \mathbf{z})$ is differentiable on $z$ almost everywhere up to the order $\alpha$. For every $0 \leq k \leq \alpha$ and every $1 \leq i_{1}, \ldots, i_{\alpha} \leq p$, let

$$
\mathcal{H}_{k, i}\left(\mathbf{u}, \mathbf{v}, \mathbf{x}_{1}, \mathbf{x}_{2}, \mathbf{z}\right):=\sup _{t \in[0,1]} \mid \frac{\partial^{k} f_{\mathbf{X}, \mathbf{Z}}}{\partial z_{i_{1}} \ldots \partial z_{i_{k}}}\left(\mathbf{x}_{1}, \mathbf{z}+t h \mathbf{u}\right) \frac{\partial^{\alpha-k} f_{\mathbf{X}, \mathbf{Z}}}{\partial z_{i_{k+1}} \ldots \partial z_{i_{\alpha}}}\left(\mathbf{x}_{2}, \mathbf{z}+\text { th } \mathbf{v}\right) \mid,
$$

denoting $\vec{\imath}=\left(i_{1}, \ldots, i_{\alpha}\right)$. Assume that $\mathcal{H}_{k, \vec{\imath}}\left(\mathbf{u}, \mathbf{v}, \mathbf{x}_{1}, \mathbf{x}_{2}, \mathbf{z}\right)$ is integrable and there exists a finite constant $C_{\mathbf{X Z}, \alpha}>$ 0 such that, for every $\mathbf{z} \in Z$ and every $h<1$,

$$
\int|K|(\mathbf{u})|K|(\mathbf{v}) \sum_{k=0}^{\alpha}\left(\begin{array}{l}
\alpha \\
k
\end{array}\right) \sum_{i_{1}, \ldots, i_{\alpha}=1}^{p} \mathcal{H}_{k, i}\left(\mathbf{u}, \mathbf{v}, \mathbf{x}_{1}, \mathbf{x}_{2}, \mathbf{z}\right)\left|u_{i_{1}} \ldots u_{i_{k}} v_{i_{k+1}} \ldots v_{i_{\alpha}}\right| d \mathbf{u} d \mathbf{v} d \mathbf{x}_{1} d \mathbf{x}_{2}
$$

is less than $C_{\mathbf{X Z}, \alpha}$.

Assumptions 3.2 and 3.4 are satisfied when the density of $\mathbf{Z}$ is $\alpha$-times continuously differentiable in a (strictly larger) neighborhood of $z$ and $K$ is compactly supported, for $n$ sufficiently large. Indeed, the vectors thu and thv will then be arbitrary small uniformly w.r.t. $t \in[0,1]$ and $\mathbf{u}$ (resp. v) in the support of $K^{3}$. If $K$ is not compactly supported, these assumptions are most often satisfied when the tails of $f_{\mathbf{Z}}$ and its derivatives do not exhibit pathological patterns. For instance, if $f_{Z}$ is a Gaussian density, this is the case because this density and its derivatives are bounded on $\mathbb{R}^{p}$.

The next three propositions state pointwise and uniform exponential inequalities for the estimators $\hat{\tau}_{1,2 \mid \mathbf{Z}=\mathbf{z}}^{(k)}$, when $k=1,2,3$. They are proved in Sections A.4, A.5 and A.6. We will denote $c_{1}:=c_{3}:=4$ and $c_{2}:=2$.

Proposition 3 (Exponential bound with explicit constants). Under Assumptions 3.1-3.4, for every $t>0$ such that $C_{K, \alpha} h^{\alpha} / \alpha !+t \leq f_{\mathbf{Z}, \min } / 2$ and every $t^{\prime}>0$, if $C_{\tilde{K}, 2} h^{2}<f_{\mathbf{z}}(\mathbf{z})$, we have

$$
\begin{aligned}
& \mathbb{P}\left(\left|\hat{\tau}_{1,2 \mid \mathbf{Z}=\mathbf{z}}^{(k)}-\tau_{1,2 \mid \mathbf{Z}=\mathbf{z}}\right|>\frac{c_{k}}{f_{\mathbf{Z}}^{2}(\mathbf{z})}\left(\frac{C_{\mathbf{X Z}, \alpha} h^{\alpha}}{\alpha !}+\frac{3 f_{\mathbf{Z}}(\mathbf{z}) \int K^{2}}{2 n h^{p}}+t^{\prime}\right) \times\left(1+\frac{16 f_{\mathbf{Z}}^{2}(\mathbf{z})}{f_{\mathbf{Z}, \min }^{3}}\left(\frac{C_{K, \alpha} h^{\alpha}}{\alpha !}+t\right)\right)\right) \\
& \leq 2 \exp \left(-\frac{n h^{p} t^{2}}{2 f_{\mathbf{Z}, \max } \int K^{2}+(2 / 3) C_{K} t}\right)+2 \exp \left(-\frac{(n-1) h^{2 p} t^{\prime 2}}{4 f_{\mathbf{Z}, \max }^{2}\left(\int K^{2}\right)^{2}+(8 / 3) C_{K}^{2} t^{\prime}}\right) \\
& +2 \exp \left(-\frac{n h^{p}\left(f_{\mathbf{z}}(\mathbf{z})-C_{\tilde{K}, 2} h^{2}\right)^{2}}{8 f_{\mathbf{Z}, \text { max }} \int \tilde{K}^{2}+4 C_{\tilde{K}}\left(f_{\mathbf{z}}(\mathbf{z})-C_{\tilde{K}, 2} h^{2}\right) / 3}\right),
\end{aligned}
$$

for any $\mathbf{z} \in Z$ and every $k=1,2,3$.

Alternatively, we can apply Theorem 1 in Major [26] instead of the Bernstein-type inequality that has been used in the proof of Proposition 3.

3 Then, all the terms that involve $f_{\mathbf{Z}}$ and its derivatives are uniformly bounded. And invoke the $\alpha$-order property of $K$ to check the validity of such assumptions. 
Proposition 4 (Alternative exponential bound without explicit constants). Under Assumptions 3.1-3.4, for every $t>0$ such that $C_{K, \alpha} h^{\alpha} / \alpha !+t \leq f_{\mathrm{Z}, \min } / 2$ and every $t^{\prime}>0$ s.t. $t^{\prime} \leq 2 h^{p}\left(\int K^{2}\right)^{3} f_{\mathrm{Z}, \text { max }}^{3} / C_{K}^{4}$, there exist some universal constants $C_{2}$ and $\alpha_{2}$ s.t.

$$
\begin{aligned}
& \mathbb{P}\left(\left|\hat{\tau}_{1,2 \mid \mathbf{Z}=\mathbf{z}}^{(k)}-\tau_{1,2 \mid \mathbf{Z}=\mathbf{z}}\right|>\frac{c_{k}}{f_{\mathbf{Z}}^{2}(\mathbf{z})}\left(\frac{C_{\mathbf{X Z}, \alpha} h^{\alpha}}{\alpha !}+\frac{3 f_{\mathbf{Z}}(\mathbf{z}) \int K^{2}}{2 n h^{p}}+t^{\prime}\right) \times\left(1+\frac{16 f_{\mathbf{Z}}^{2}(\mathbf{z})}{f_{\mathbf{Z}, \min }^{3}}\left(\frac{C_{K, \alpha} h^{\alpha}}{\alpha !}+t\right)\right)\right) \\
& \leq 2 \exp \left(-\frac{n h^{p} t^{2}}{2 f_{\mathbf{Z}, \max } \int K^{2}+(2 / 3) C_{K} t}\right)+2 \exp \left(-\frac{n h^{p}\left(f_{\mathbf{z}}(\mathbf{z})-C_{\tilde{K}, 2} h^{2}\right)^{2}}{8 f_{\mathbf{Z}, \max } \int \tilde{K}^{2}+4 C_{\tilde{K}}\left(f_{\mathbf{z}}(\mathbf{z})-C_{\tilde{K}, 2} h^{2}\right) / 3}\right) \\
& +2 \exp \left(\frac{n h^{p} t^{2}}{32 \int K^{2}\left(\int|K|\right)^{2} f_{\mathbf{Z}, \text { max }}^{3}+8 C_{K} \int|K| f_{\mathbf{Z}, \text { max }} t / 3}\right)+C_{2} \exp \left(-\frac{\alpha_{2} n h^{p} t^{\prime}}{8 f_{\mathbf{Z}, \max }\left(\int K^{2}\right)}\right),
\end{aligned}
$$

for any $\mathbf{z} \in Z$ and every $k=1,2,3$, if $C_{\tilde{K}, 2} h^{2}<f_{\mathbf{Z}}(\mathbf{z})$ and $6 h^{p}\left(\int|K|\right)^{2} f_{\mathbf{z}, \max }<\int K^{2}$.

Remark 5. In Propositions 2,3 and 4 , when the support of $K$ is included in $[-c, c]^{p}$ for some $c>0, f_{\mathrm{Z}, \max }$ can be replaced by a local bound $\sup _{\tilde{\mathbf{z}} \in \mathcal{V}(\mathbf{z}, \epsilon)} f_{\mathbf{Z}}(\tilde{\mathbf{z}})$, denoting by $\mathcal{V}(\mathbf{z}, \epsilon)$ a closed ball of center $\mathbf{z}$ and any radius $\epsilon>0$, when $h c<\epsilon$.

Propositions 3 and 4 look similar. Nonetheless, only the upper bound in the former case can be explicitly calculated because this bound involves constants that can be numerically evaluated. But, on the other side, Proposition 4 provides better rates of convergence. Indeed, by choosing $t^{\prime}$ of the order $h^{p}$, the latter result can be summarized as $\mathbb{P}\left(\left|\hat{\tau}_{1,2 \mid \mathbf{Z}=\mathbf{z}}^{(k)}-\tau_{1,2 \mid \mathbf{Z}=\mathbf{z}}\right|>\epsilon h^{p}\right) \asymp \exp \left(-C n h^{2 p} \epsilon\right)$, for some constants $\epsilon>0$ and $C>0$. At the opposite, the bound obtained in Proposition 3 is of the type $\mathbb{P}\left(\left|\hat{\tau}_{1,2 \mid \mathbf{Z}=\mathbf{z}}^{(k)}-\tau_{1,2 \mid \mathbf{Z}=\mathbf{z}}\right|>\epsilon\right) \asymp \exp \left(-C^{\prime} n h^{2 p} \epsilon\right)$, $C^{\prime}>0$, what is clearly weaker.

As a corollary, the two latter results yield the weak consistency of $\hat{\tau}_{1,2 \mid \mathbf{Z}=\mathbf{z}}^{(k)}$ for every $\mathbf{z} \in \mathcal{Z}$, when $n h^{2 p} \rightarrow \infty$ (choose the constants $t$ and $t^{\prime} \sim h^{p}$ sufficiently small, in Proposition 4, e.g.).

It is possible to obtain uniform bounds, by slightly strengthening our assumptions. Note that this next result will be true if $n$ is sufficiently large, when Proposition 4 was true for every $n$.

Assumption 3.5. The kernel $K$ is Lipschitz on $\left(Z,\|\cdot\|_{\infty}\right)$, with a constant $\lambda_{K}$ and $Z$ is a subset of an hypercube in $\mathbb{R}^{p}$ whose volume is denoted by $\mathcal{V}$. Moreover, $K$ and $K^{2}$ are regular in the sense of [16] or [11].

Proposition 6 (Uniform exponential bound). Under the assumptions 3.1-3.5, there exist some constants $L_{K}$ and $\bar{C}_{K}\left(\right.$ resp. $L_{\tilde{K}}$ and $\left.\bar{C}_{\tilde{K}}\right)$ that depend only on the VC characteristics of $K($ resp. $\tilde{K})$, s.t., for every $\mu \in(0,1)$ such that $\mu f_{\mathbf{Z}, \min }<C_{\mathbf{X Z}, \alpha} h^{\alpha} / \alpha !+b_{K} \int K^{2} f_{\mathbf{Z}, \max } / C_{K}$, if $f_{\mathbf{Z}, \text { max }}<\tilde{C}_{\mathbf{X Z}, 2} h^{2} / 2+b_{\tilde{K}} \int \tilde{K}^{2} f_{\mathbf{Z}, \text { max }} / C_{\tilde{K}}$,

$$
\begin{aligned}
& \mathbb{P}\left(\sup _{\mathbf{z} \in \mathcal{Z}}\left|\hat{\tau}_{1,2 \mid \mathbf{Z}=\mathbf{z}}^{(k)}-\tau_{1,2 \mid \mathbf{Z}=\mathbf{z}}\right|>\frac{c_{k}}{f_{\mathbf{z}, \min }^{2}(1-\mu)^{2}}\left(\frac{C_{\mathbf{X Z}, \alpha} h^{\alpha}}{\alpha !}+\frac{3 f_{\mathbf{z}, \max } \int K^{2}}{2 n h^{p}}+t\right)\right) \\
& \quad \leq \quad L_{K} \exp \left(-C_{f, K} n h^{p}\left(\mu f_{\mathbf{z}, \min }-\frac{C_{\mathbf{X Z}, \alpha} h^{\alpha}}{\alpha !}\right)^{2}\right) \\
& \quad+\quad C_{2} D \exp \left(-\frac{\alpha_{2} n t h^{p}}{8 f_{\mathbf{Z}, \max }\left(\int K^{2}\right)}\right)+L_{\tilde{K}} \exp \left(-C_{f, \tilde{K}} n h^{p}\left(f_{\mathbf{z}, \max }-\tilde{C}_{\mathbf{X Z}, 2} h^{2}\right)^{2} / 4\right) \\
& \quad+\quad 2 \exp \left(-\frac{A_{2} n h^{p} t^{2} C_{K}^{-4}}{16^{2} A_{1}^{2} \int K^{2} f_{\mathbf{z}, \max }^{3}\left(\int|K|\right)^{2}}\right)+2 \exp \left(-\frac{A_{2} n h^{p} t}{16 C_{K}^{2} A_{1}}\right),
\end{aligned}
$$

for $n$ sufficiently large, $k=1,2,3$, and for every $t>0$ s.t. $t \leq 2 h^{p}\left(\int K^{2}\right)^{3} f_{\mathbf{Z}, \max }^{3} / C_{K}^{4}$,

$$
\begin{gathered}
-16 A_{1} C_{K}^{2} A_{\bar{g}} \int K^{2} f_{\mathbf{z}, \max }^{3}\left(\int|K|\right)^{2} \ln \left(h^{p} \int K^{2} f_{\mathbf{z}, \max }^{3}\left(\int|K|\right)^{2}\right)<n^{1 / 2} h^{p / 2} t, \text { and } \\
n h^{p} t \geq\left(\int K^{2}\right) f_{\mathbf{z}, \max } M_{2}(p+\beta)^{3 / 2} \log \left(\frac{4 C_{K}^{2}}{h^{p} f_{\mathbf{z}, \max } \int K^{2}}\right), \beta=\max \left(0, \frac{\log D}{\log n}\right), D:=\left\lceil\mathcal{V}\left(\frac{4 C_{K} \lambda_{K}}{h}\right)^{p}\right\rceil,
\end{gathered}
$$

for some universal constants $C_{2}, \alpha_{2}, M_{2}, A_{1}, A_{2}$ and a constant $A_{\bar{g}}$ that depends on $K$ and $f_{\mathbf{z}, \max }$. 
We have denoted $C_{f, K}:=\log \left(1+b_{K} /\left(4 L_{K}\right)\right) /\left(L_{K} b_{K} f_{\mathbf{z}, \max } \int K^{2}\right)$, for any arbitrarily chosen constant $b_{K} \geq \bar{C}_{K}$. Similarly, $C_{f, \tilde{K}}:=\log \left(1+b_{\tilde{K}} /\left(4 L_{\tilde{K}}\right)\right) /\left(L_{\tilde{K}} b_{\tilde{K}} f_{\mathbf{z}, \max } \int \tilde{K}^{2}\right), b_{\tilde{K}} \geq \bar{C}_{\tilde{K}}$.

\subsection{Asymptotic behavior}

The previous exponential inequalities are not optimal to prove usual asymptotic results. Indeed, they directly or indirectly rely on upper bounds of estimates, as in Hoeffding or Bernstein-type inequalities. In the case of kernel estimates, this implies the necessary condition $n h^{2 p} \rightarrow \infty$, at least. By a direct approach, it is possible to state the consistency of $\hat{\tau}_{1,2 \mid \mathbf{Z}=\mathbf{z}}^{(k)}, k=1,2,3$, and then of $\tilde{\tau}_{1,2 \mid \mathbf{Z}=\mathbf{z}}$, under the weaker condition $n h^{p} \rightarrow \infty$.

Proposition 7 (Consistency). Under Assumption 3.1, if $n h_{n}^{p} \rightarrow \infty, \lim K(\mathbf{t})|\mathbf{t}|^{p}=0$ when $|\mathbf{t}| \rightarrow \infty$, $f_{\mathrm{Z}}$ and $\mathbf{z} \mapsto \tau_{1,2 \mid \mathbf{Z}=\mathbf{z}}$ are continuous on $z$, then $\hat{\tau}_{1,2 \mid \mathbf{Z}=\mathbf{z}}^{(k)}$ tends to $\tau_{1,2 \mid \mathbf{Z}=\mathbf{z}}$ in probability, when $n \rightarrow \infty$ for any $k=1,2,3$.

This property is proved in A.7. Moreover, Proposition 6 does not allow to state the strong uniform consistency of $\hat{\tau}_{1,2 \mid \mathbf{Z}=\mathbf{z}}^{(k)}$ because the threshold $t$ has to be of order $h^{p}$ at most. Here again, a direct approach is possible, nonetheless.

Proposition 8 (Uniform consistency). Under Assumption 3.1, assume that $n h_{n}^{2 p} / \log n \rightarrow \infty, \lim K(\mathbf{t})|\mathbf{t}|^{p}=0$ when $|\mathbf{t}| \rightarrow \infty, K$ is Lipschitz, $f_{\mathbf{Z}}$ and $\mathbf{z} \mapsto \tau_{1,2 \mid \mathbf{Z}=\mathbf{z}}$ are continuous on a bounded set $Z$, and there exists a lower bound $f_{\mathbf{Z} \text {,min }}$ s.t. $f_{\mathbf{Z}, \min } \leq f_{\mathbf{Z}}(\mathbf{z})$ for any $\mathbf{z} \in \mathcal{Z}$. Then $\sup _{\mathbf{z} \in \mathcal{Z}}\left|\hat{\tau}_{1,2 \mid \mathbf{Z}=\mathbf{z}}^{(k)}-\tau_{1,2 \mid \mathbf{Z}=\mathbf{z}}\right| \rightarrow 0$ almost surely, when $n \rightarrow \infty$ for any $k=1,2,3$.

This property is proved in A.8. To derive the asymptotic law of this estimator, we will assume:

Assumption 3.6. (i) $n h_{n}^{p} \rightarrow \infty$ and $n h_{n}^{p+2 \alpha} \rightarrow 0$; (ii) $K(\cdot)$ is compactly supported.

Proposition 9 (Joint asymptotic normality at different points). Let $\mathbf{z}_{1}^{\prime}, \ldots, \mathbf{z}_{n^{\prime}}^{\prime}$ be fixed points in a set $z \subset \mathbb{R}^{p}$. Assume 3.1, 3.4, 3.6, that the $\mathbf{z}_{i}^{\prime}$ are distinct and that $f_{\mathbf{Z}}$ and $\mathbf{z} \mapsto f_{\mathbf{X}, \mathbf{Z}}(\mathbf{x}, \mathbf{z})$ are continuous on $z$, for every $\mathbf{x}$. Then, as $n \rightarrow \infty$,

$$
\left(n h_{n}^{p}\right)^{1 / 2}\left(\hat{\tau}_{1,2 \mid \mathbf{Z}=\mathbf{z}_{i}^{\prime}}-\tau_{1,2 \mid \mathbf{Z}=\mathbf{z}_{i}^{\prime}}\right)_{i=1, \ldots, n^{\prime}} \stackrel{D}{\longrightarrow} \mathcal{N}\left(0, \mathbb{H}^{(k)}\right), k=1,2,3,
$$

where $\hat{\tau}_{1,2 \mid \mathbf{Z}=\mathbf{z}}$ denotes any of the estimators $\hat{\tau}_{1,2 \mid \mathbf{Z}=\mathbf{z}}^{(k)}, k=1,2,3$ or $\tilde{\tau}_{1,2 \mid \mathbf{Z}=\mathbf{z}}$, and $\mathbb{H}$ is the $n^{\prime} \times n^{\prime}$ diagonal real matrix defined by

$$
\left[\mathbb{H}^{(k)}\right]_{i, j}=\frac{4 \int K^{2} \mathbb{1}_{\{i=j\}}}{f_{\mathbf{Z}}\left(\mathbf{z}_{i}^{\prime}\right)}\left\{\mathbb{E}\left[g_{k}\left(\mathbf{X}_{1}, \mathbf{X}\right) g_{k}\left(\mathbf{X}_{2}, \mathbf{X}\right) \mid \mathbf{Z}=\mathbf{Z}_{1}=\mathbf{Z}_{2}=\mathbf{z}_{i}^{\prime}\right]-\tau_{1,2 \mid \mathbf{Z}=\mathbf{z}_{i}^{\prime}}^{2}\right\},
$$

for every $1 \leq i, j \leq n^{\prime}$, and $(\mathbf{X}, \mathbf{Z}),\left(\mathbf{X}_{1}, \mathbf{Z}_{1}\right),\left(\mathbf{X}_{2}, \mathbf{Z}_{2}\right)$ are independent versions.

This proposition is proved in A.9.

Remark 10. The latter results will provide some simple tests of the constancy of the function $\mathbf{z} \mapsto \tau_{1,2 \mid \mathbf{z}}$, and then of the constancy of the associated conditional copula itself. This would test the famous "simplifying assumption" (" $\mathcal{H}_{0}: C_{1,2 \mid \mathbf{Z}=\mathbf{z}}$ does not depend on the choice of $\mathbf{z}$ "), a key assumption for vine modeling in particular: see [1] or [17] for a discussion, [8] for a review and a presentation of formal tests for this hypothesis.

\section{Simulation study}

In this simulation study, we draw i.i.d. random samples $\left(X_{i, 1}, X_{i, 2}, Z_{i}\right), i=1, \ldots, n$, with univariate explanatory variables $(p=1)$. We consider two settings, that correspond to bounded and/or unbounded explanatory variables respectively: 
1. $z=] 0,1\left[\right.$ and the law of $Z$ is uniform on ]0, 1[. Conditionally on $Z=z, X_{1} \mid Z=z$ and $X_{2} \mid Z=z$ both follow a Gaussian distribution $\mathcal{N}(z, 1)$. Their associated conditional copula is Gaussian and their conditional Kendall's tau is given by $\tau_{1,2 \mid Z=z}=2 z-1$.

2. $z=$ ]-2, 2[ and the law of $Z$ is $\mathcal{N}(0,1)$. Conditionally on $Z=z, X_{1} \mid Z=z$ and $X_{2} \mid Z=z$ both follow a Gaussian distribution $\mathcal{N}(\Phi(z), 1)$, where $\Phi(\cdot)$ is the cdf of the $\mathbf{Z}$. Their associated conditional copula is Gaussian and their conditional Kendall's tau is given by $\tau_{1,2 \mid Z=z}=2 \Phi(z)-1$.

These simple frameworks allow us to compare the numerical properties of our different estimators in different parts of the space, in particular when $Z$ is close to zero or one, i.e. when the conditional Kendall's tau is close to -1 or to 1 . Note that these distributions are continuous, with infinitely differentiable densities. We will use the Epanechnikov kernel. Therefore, they will satisfy Assumptions 3.1-3.6. We compute the different estimators $\hat{\tau}_{1,2 \mid \mathbf{Z}=\mathbf{z}}^{(k)}$ for $k=1,2,3$, and the symmetrically rescaled version $\tilde{\tau}_{1,2 \mid z}$. The bandwidth $h$ is chosen as proportional to the usual "rule-of-thumb" for kernel density estimation, i.e. $h=\alpha_{h} \hat{\sigma}(Z) n^{-1 / 5}$ with $\alpha_{h} \in$ $\{0.5,0.75,1,1.5,2\}$ and $n \in\{100,500,1000,2000\}$. For each setting, we consider three local measures of goodness-of-fit: for a given $z$ and for any Kendall's tau estimate (say $\hat{\tau}_{1,2 \mid Z=z}$ ), let

- the (local) bias: $\operatorname{Bias}(z):=\mathbb{E}\left[\hat{\tau}_{1,2 \mid Z=z}\right]-\tau_{1,2 \mid Z=z}$,

- the (local) standard deviation: $\operatorname{Sd}(z):=\mathbb{E}\left[\left(\hat{\tau}_{1,2 \mid Z=z}-\mathbb{E}\left[\hat{\tau}_{1,2 \mid Z=z}\right]\right)^{2}\right]^{1 / 2}$,

- the (local) mean square-error: $\operatorname{MSE}(z):=\mathbb{E}\left[\left(\hat{\tau}_{1,2 \mid Z=z}-\tau_{1,2 \mid Z=z}\right)^{2}\right]$.

We also consider their integrated version w.r.t the usual Lebesgue measure on the whole support of $z$, respectively denoted by IBias, ISd and IMSE. Some results concerning these integrated measures are given in Table 1 (resp. Table 2) for Setting 1 (resp. Setting 2), and for different choices of $\alpha_{h}$ and $n$. For the sake of effective calculations of these measures, all the theoretical previous expectations are replaced by their empirical counterparts based on 500 simulations.

For every $n$, the best results seem to be obtained with $\alpha_{h}=1.5$ and the fourth (rescaled) estimator, particularly in terms of bias. This is not so surprising, because the estimators $\hat{\tau}^{(k)}, k=1,2,3$, do not have the right support at a finite distance. Note that this comparative advantage of $\tilde{\tau}$ in terms of bias decreases with $n$, as expected. In terms of integrated variance, all the considered estimators behave more or less similarly, particularly when $n \geq 500$.

To illustrate our results for Setting 1 (resp. Setting 2), the functions $z \mapsto \operatorname{Bias}(z), \operatorname{Sd}(z)$ and $\operatorname{MSE}(z)$ have been plotted on Figures 1-2 (resp. Figures 3-4), both with our empirically optimal choice $\alpha_{h}=1.5$. We can note that, considering the bias, the estimator $\tilde{\tau}$ behaves similarly as $\hat{\tau}^{(1)}$ when the true $\tau$ is close to -1 , and similarly as $\hat{\tau}^{(3)}$ when the true Kendall's tau is close to 1 . But globally, the best pointwise estimator is clearly obtained with the rescaled version $\tilde{\tau}_{1,2 \mid \mathrm{Z}=\text {., }}$ after a quick inspection of MSE levels, and even if the differences between our four estimators weaken for large sample sizes. The comparative advantage of $\tilde{\tau}_{1,2 \mid \mathbf{z}}$ more clearly appears with Setting 2 than with Setting 1. Indeed, in the former case, the support of Z's distribution is the whole line. Then $\hat{f}_{\mathrm{Z}}$ does not suffer any more from the boundary bias phenomenon, contrary to what happened with Setting 1 . As a consequence, the biases induced by the definitions of $\hat{\tau}_{1,2 \mid \mathbf{z}}^{(k)}, k=1,3$, appear more strikingly in Figure 3, for instance: when $z$ is close to (-1) (resp. 1), the biases of $\hat{\tau}_{1,2 \mid z}^{(1)}$ (resp. $\hat{\tau}_{1,2 \mid z}^{(3)}$ ) and $\tilde{\tau}_{1,2 \mid z}$ are close, when the bias $\hat{\tau}_{1,2 \mid z}^{(3)}$ (resp. $\hat{\tau}_{1,2 \mid z}^{(1)}$ ) is a lot larger. Since the squared biases are here significantly larger than the variances in the tails, $\tilde{\tau}_{1,2 \mid z}$ provides the best estimator globally considering "both sides" together. But even in the center of Z's distribution, the latter estimator behaves very well.

In Setting 2 where there is no boundary problem, we also try to estimate the conditional Kendall's tau using our cross-validation criterion (4), with $N_{\text {pairs }}=1000$. More precisely, denoting by $h^{C V}$ the minimizer of the cross-validation criterion, we try different choices $h=\alpha_{h} \times h^{C V}$ with $\alpha_{h} \in\{0.5,0.75,1,1.5,2\}$. The results in terms of integrated bias, standard deviation and MSE are given in Table 3. We do not find any substantial improvements compared to the previous Table 2, where the bandwidth was chosen "roughly". In Table 4, 
we compare the average $h^{C V}$ with the previous choice of $h$. The expectation of $h^{C V}$ is always higher than the "rule-of-thumb" $h^{\text {ref }}$, but the difference between both decreases when the sample size $n$ increases. The standard deviation of $h^{C V}$ is quite high for low values of $n$, but decreases as a function of $n$. This may be seen as quite surprising given the fact that the number of pairs $N_{\text {pairs }}$ used in the computation of the criterion stays constant. Nevertheless, when the sample size increases, the selected pairs are better in the sense that the differences $\left|Z_{i}-Z_{j}\right|$ can become smaller as more replications of $Z_{i}$ are available.

Table 1: Results of the simulation in Setting 1. All values have been multiplied by 1000. Bold values indicate optimal choices for the chosen measure of performance. These results are integrated measures of performance over the whole space $z$; the corresponding local measures of performance are displayed in Figures 1 and 2.

\begin{tabular}{|c|c|c|c|c|c|c|c|c|c|c|c|c|c|}
\hline \multirow{6}{*}{$\begin{array}{l}0 \\
11 \\
\tilde{8}\end{array}$} & \multirow{6}{*}{$\begin{array}{l}\hat{\tau}_{1,2 \mid \mathbf{Z}=\cdot}^{(1)} \\
\hat{\tau}_{1,2 \mid \mathbf{Z}=\cdot}^{(2)} \\
\hat{\tau}_{1,2 \mid \mathbf{Z}=\cdot}^{(3)} \\
\tilde{\tau}_{1,2 \mid \mathbf{Z}==} .\end{array}$} & \multicolumn{3}{|c|}{$=100$} & \multicolumn{3}{|c|}{$n=500$} & \multicolumn{3}{|c|}{$n=1000$} & \multicolumn{3}{|c|}{$n=2000$} \\
\hline & & ias & $15 \mathrm{~d}$ & IMSE & & Sd & IMSE & Bias & ISd & IMSF & IBias & ISd & IMSE \\
\hline & & -133 & 197 & 66.5 & -34.5 & 84.9 & 9.86 & -18.2 & 61.6 & 4.85 & -10.9 & 46 & 2.65 \\
\hline & & 12.9 & 187 & 43.7 & -4.08 & 84.4 & 8.58 & -0.9 & 61.5 & 4.49 & -1.07 & 46 & 2.53 \\
\hline & & 107 & 190 & 56.6 & 26.4 & 84.5 & 9.26 & 16.4 & 61.5 & 4.76 & 8.8 & 46 & 2.6 \\
\hline & & -0.91 & 213 & 48.2 & -1.18 & 86.9 & 8.55 & 0.733 & 62.4 & 4.46 & -0.149 & 46.4 & .5 \\
\hline \multirow{4}{*}{$\begin{array}{l}\hat{0} \\
11 \\
\tilde{8}\end{array}$} & $\hat{\tau}_{1,2 \mid \mathbf{Z}=}^{(1)}$ & -88 & 150 & 35.8 & 263 & 68 & 6.32 & -13.9 & 50.7 & 3.33 & -7.98 & 37.6 & 1.8 \\
\hline & $\hat{\tau}_{1,2 \mid \mathbf{Z}=\cdot}^{(2)}$ & -10.4 & 145 & 26.3 & 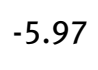 & 67.9 & & -2.33 & 50.6 & & -1.39 & 37.5 & 1.74 \\
\hline & $\hat{\tau}_{1,2 \mid \mathbf{Z}=}^{(3)}$ & 67.2 & 146 & 30.6 & 14.3 & 67.9 & 5.75 & 9.2 & 50.6 & 3.19 & 5.2 & 37.5 & 1.76 \\
\hline & $\tilde{\boldsymbol{\tau}}_{1,2 \mid \mathbf{Z}=}$ & -2.06 & זנד & 26.7 & 1.3. & 69.2 & 0.48 & -1.21 & 51.2 & נט.0 & -0.76 & 37.8 & 1.69 \\
\hline \multirow{4}{*}{$\begin{array}{l}\vec{H} \\
\overrightarrow{8}\end{array}$} & $\hat{\tau}_{1,2 \mid \mathbf{Z}=.}^{(1)}$ & -67.8 & 12 & 24. & 19.2 & 58.7 & & & 43.1 & 2.5 & -6.34 & 3 & 1.44 \\
\hline & $\hat{\tau}_{1,2 \mid \mathbf{Z}=\cdot}^{(2)}$ & -9.99 & 121 & 19 & -3.95 & 58.6 & 4.39 & -2.35 & 43.1 & 2.39 & -1.39 & 33 & 1.4 \\
\hline & $\hat{\boldsymbol{\tau}}_{1,2 \mid \mathbf{Z}=\cdot}^{(3)}$ & 47.8 & 122 & 20.9 & 11.3 & 58.7 & 4.47 & 6.34 & 43.1 & 2.41 & 3.57 & 33 & 1.41 \\
\hline & $\tilde{\tau}_{1,2 \mid \mathbf{Z}=}$ & -3.48 & 128 & & 23 & & & 116 & & & -0.897 & & 1.35 \\
\hline \multirow{4}{*}{ 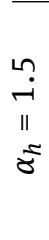 } & $\hat{\tau}_{1,2 \mid \mathbf{Z}=\cdot}^{(1)}$ & -44.6 & 101 & 17.5 & -13.9 & 50.4 & 4.12 & -9.7 & 35.9 & 2.13 & -5.52 & 27.6 & 1.28 \\
\hline & $\hat{\tau}_{1,2 \mid \mathbf{Z}=\cdot}^{(2)}$ & -5.81 & 100 & 14.9 & -5.68 & 50.3 & 3.84 & -3.84 & 35.9 & 2.02 & -2.18 & 27.6 & 1.24 \\
\hline & $\hat{\tau}_{1,2 \mid \mathbf{Z}=\cdot}^{(3)}$ & 33 & 101 & & & 50.3 & & 2.01 & 35.9 & 1.99 & & 27.6 & 1.23 \\
\hline & $\tilde{\boldsymbol{\tau}}_{1,2 \mid \mathbf{Z}=}$ & -1.09 & 104 & 13.4 & -4.55 & 50.8 & 3.57 & -3.19 & 36.1 & & -1.83 & 27.7 & 1.18 \\
\hline \multirow{4}{*}{$\begin{array}{l}N \\
\| \\
\tilde{8}\end{array}$} & $\hat{\tau}_{1,2 \mid \mathbf{Z}=\cdot}^{(1)}$ & -37.8 & 91.4 & 17.3 & -118 & 43.8 & 4.14 & -7.2 & 31.2 & 2.35 & -5.97 & 23.7 & 1.43 \\
\hline & $\hat{\tau}_{1,2 \mid \mathbf{Z}=}^{(2)}$ & -8.03 & 10.4 & 15.4 & -3.93 & 43.8 & 3.94 & -2.75 & 31.2 & 2.28 & -3.44 & 23.7 & 1.39 \\
\hline & $\hat{\tau}_{1,2 \mid \mathbf{Z}=}^{(3)}$ & 21.7 & 91.7 & 15.4 & 3.91 & 43.8 & 3.87 & 1.7 & 31.2 & 2.24 & -0.912 & 23.7 & 1.37 \\
\hline & $\tilde{\boldsymbol{\tau}}_{1,2 \mid \mathbf{Z}=}$ & -4.5 & 94.2 & 13.5 & -3.01 & 44.1 & 3.62 & -2.24 & 31.3 & 2.12 & -3.16 & 23.8 & 1.32 \\
\hline
\end{tabular}

\section{Application to real data}

In this section, we present an application of this methodology to the dependence between electricity consumption and temperature. The first paper on this topic dates back to 1958 ([7]). Using UK data, they show that a decrease in temperature increases the electricity demand. Moreover, they show that the marginal effect of temperature levels on electricity consumption differs depending on the time of the day. Numerous other 

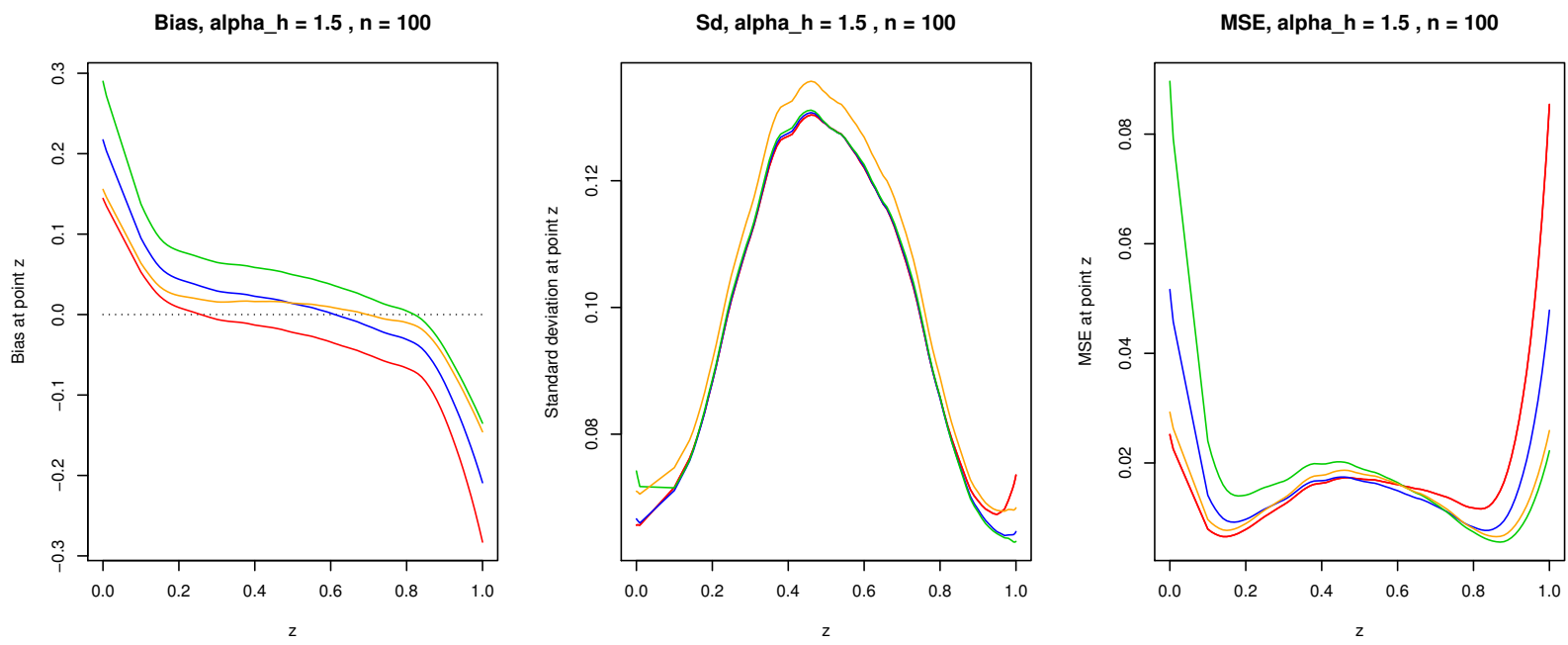

Figure 1: Local bias, standard deviation and MSE for the estimators $\hat{\tau}^{(1)}$ (red), $\hat{\tau}^{(2)}$ (blue), $\hat{\tau}^{(3)}$ (green), $\tilde{\tau}$ (orange), with $n=100$ and $\alpha_{h}=1.5$ in Setting 1 . The dotted line on the first figure is the reference at 0 .
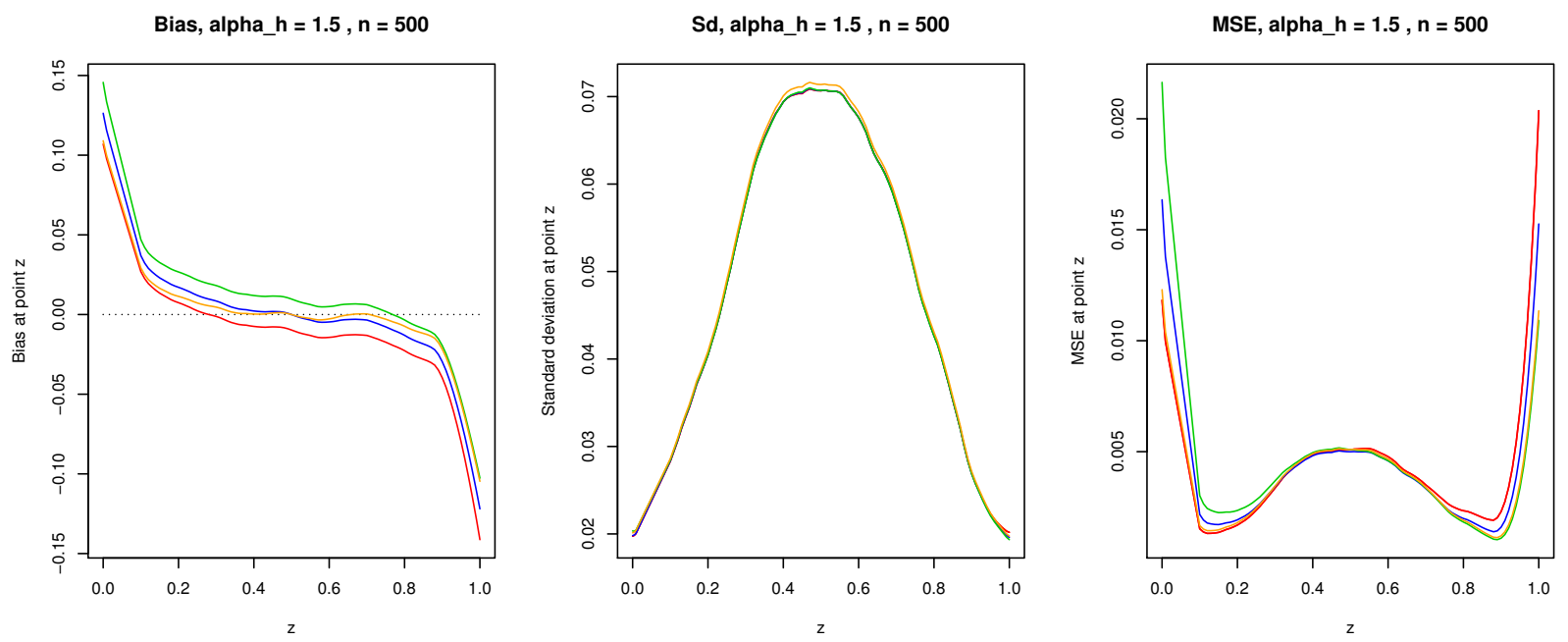

Figure 2: Local bias, standard deviation and MSE for the estimators $\hat{\tau}^{(1)}$ (red), $\hat{\tau}^{(2)}$ (blue), $\hat{\tau}^{(3)}$ (green), $\tilde{\tau}$ (orange), with $n=500$ and $\alpha_{h}=1.5$ in Setting 1. The dotted line on the first figure is the reference at 0 . 

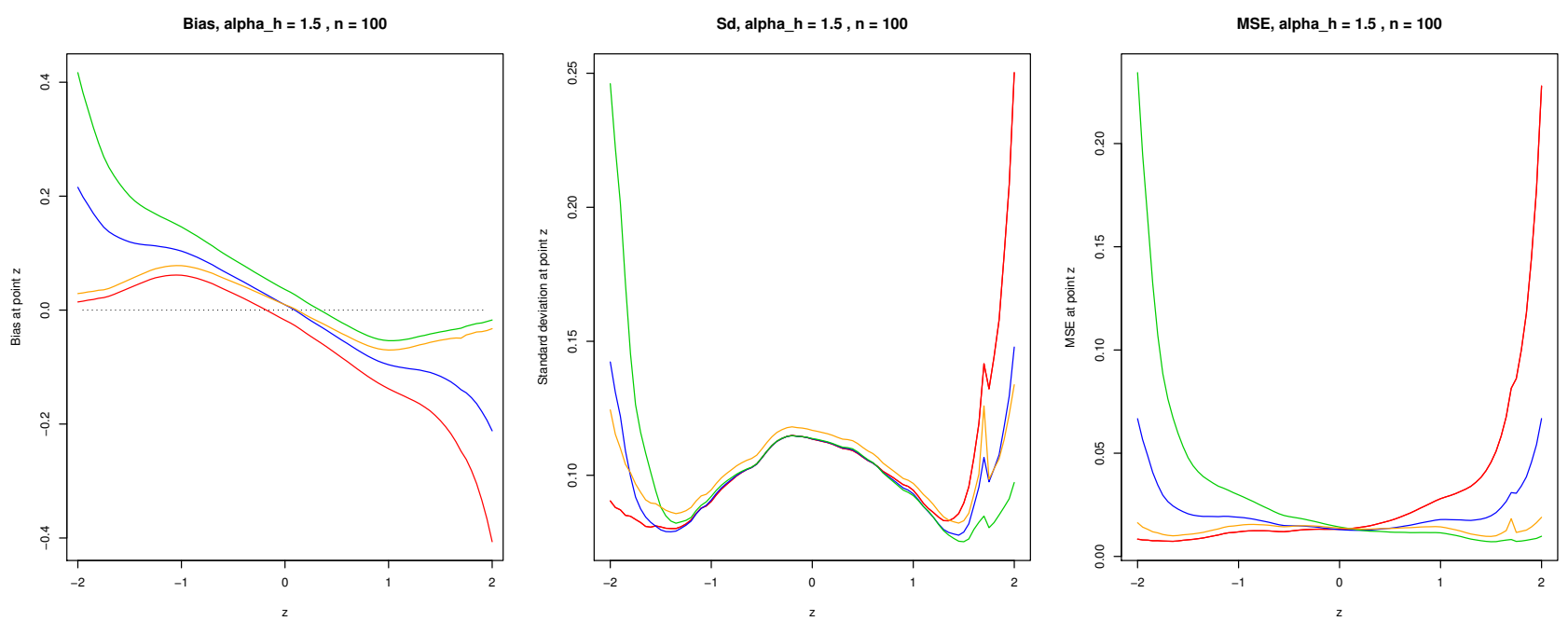

Figure 3: Local bias, standard deviation and MSE for the estimators $\hat{\tau}^{(1)}$ (red), $\hat{\tau}^{(2)}$ (blue), $\hat{\tau}^{(3)}$ (green), $\tilde{\tau}$ (orange), with $n=100$ and $\alpha_{h}=1.5$ in Setting 2. The dotted line on the first figure is the reference at 0 .
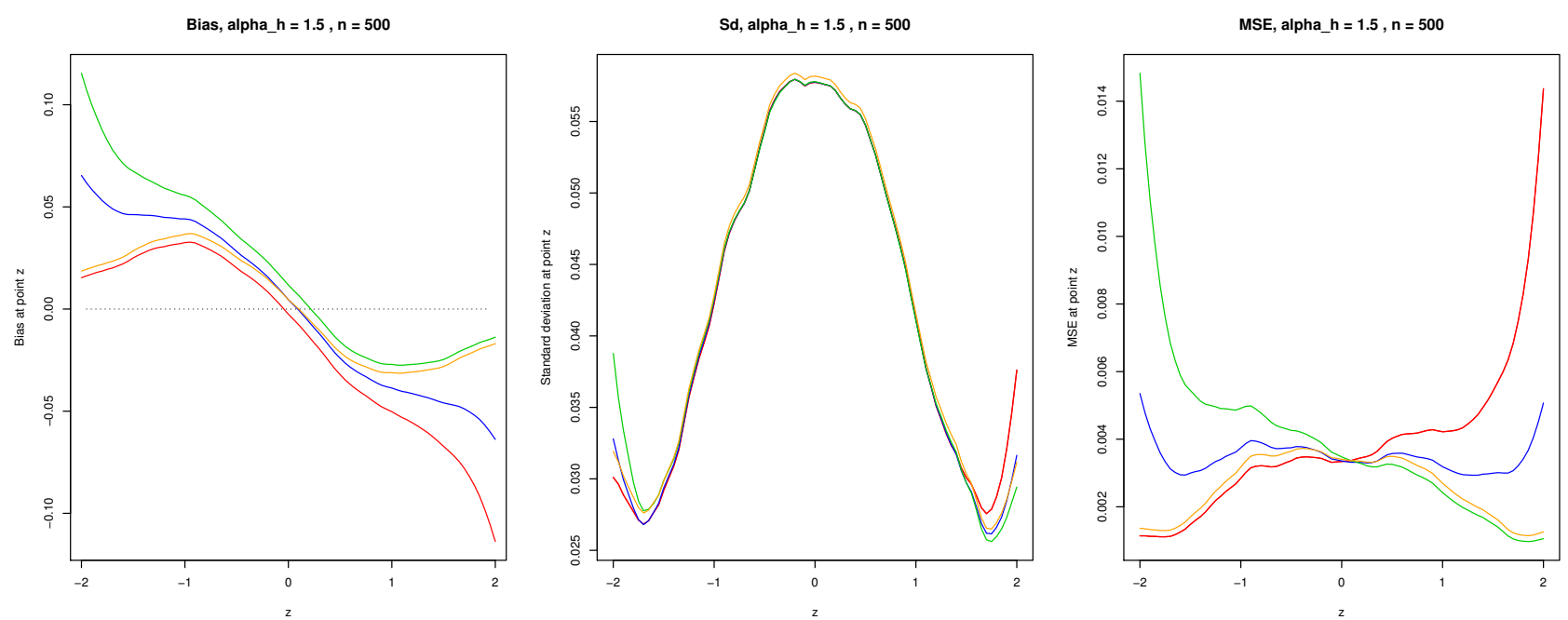

Figure 4: Local bias, standard deviation and MSE for the estimators $\hat{\tau}^{(1)}$ (red), $\hat{\tau}^{(2)}$ (blue), $\hat{\tau}^{(3)}$ (green), $\tilde{\tau}$ (orange), with $n=500$ and $\alpha_{h}=1.5$ in Setting 2 . The dotted line on the first figure is the reference at 0 . 
Table 2: Results of the simulation in Setting 2. All values have been multiplied by 1000 . Bold values indicate optimal choices for the chosen measure of performance. These results are integrated measures of performance over the whole space $Z$; the corresponding local measures of performance are displayed in Figures 3 and 4.

\begin{tabular}{|c|c|c|c|c|c|c|c|c|c|c|c|c|c|}
\hline & & \multicolumn{3}{|c|}{$n=100$} & \multicolumn{3}{|c|}{$n=500$} & \multicolumn{3}{|c|}{$n=1000$} & \multicolumn{3}{|c|}{$n=2000$} \\
\hline & \multirow{5}{*}{$\begin{array}{l}\hat{\tau}_{1,2 \mid \mathbf{Z}=\cdot}^{(1)} \\
\hat{\tau}_{1,2 \mid \mathbf{Z}=\cdot}^{(2)} \\
\hat{\tau}_{1,2 \mid \mathbf{Z}=}^{(3)} \\
\tilde{\tau}_{1,2 \mid \mathbf{Z}=}\end{array}$} & IBias & ISd & IMSE & IBias & ISd & IMSE & IBias & ISd & IMSE & IBias & ISd & IMSE \\
\hline & & -207 & 227 & 180 & -54.1 & 83.9 & 16.9 & -29.6 & 55.3 & 5.81 & -16.9 & 38.9 & 2.49 \\
\hline & & 1.15 & 207 & 97 & 0.845 & 80.5 & 10.8 & 0.557 & 54.4 & 4.35 & 0.145 & 38.6 & 2.04 \\
\hline & & 210 & 228 & 181 & 55.7 & 83.2 & 16.4 & 30.7 & 55.4 & 5.9 & 17.2 & 38.9 & 2.5 \\
\hline & & 1.4 & 225 & 51.9 & 0.987 & 81.4 & 6.86 & 0.456 & 55 & 3.22 & 0.175 & 38.9 & 1.66 \\
\hline \multirow{4}{*}{ 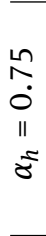 } & $\hat{\tau}_{1,2 \mid \mathbf{Z}=\cdot}^{(1)}$ & -144 & 175 & 98.6 & -33.3 & 60.6 & 7.5 & -19.8 & 41.9 & 3.12 & -10.6 & 30.5 & 1.42 \\
\hline & $\hat{\tau}_{1,2 \mid \mathbf{Z}=}^{(2)}$ & -2.33 & 163 & 56.2 & 1.73 & 59.4 & 5.56 & -0.0619 & 41.7 & 2.51 & 0.665 & 30.4 & 1.24 \\
\hline & $\hat{\tau}_{1,2 \mid \mathbf{Z}=}^{(3)}$ & 140 & 176 & 99.2 & 36.8 & 60.7 & 7.73 & 19.7 & 42.1 & 3.12 & 11.9 & 30.5 & 1.45 \\
\hline & $\tilde{\tau}_{1,2 \mid \mathbf{Z}=}$ & -3.15 & 170 & 30.3 & 1.69 & 60.2 & 3.85 & -0.093 & 42.1 & 1.95 & 0.645 & 30.5 & 1.05 \\
\hline \multirow{4}{*}{$\begin{array}{l}-1 \\
11 \\
\tilde{\sigma}\end{array}$} & $\hat{\tau}_{1,2 \mid \mathbf{Z}=.}^{(1)}$ & -99.8 & 143 & 57.7 & -24.9 & 50.9 & 5.06 & -13.5 & 36.6 & 2.28 & -6.92 & 26.6 & 1.09 \\
\hline & $\hat{\tau}_{1,2 \mid \mathbf{Z}=}^{(2)}$ & 1.17 & 132 & 34.6 & 0.903 & 50.4 & 4.02 & 1.16 & 36.5 & 1.97 & 1.46 & 26.6 & 0.994 \\
\hline & $\hat{\tau}_{1,2 \mid \mathbf{Z}=}^{(3)}$ & 102 & 139 & 54.4 & 26.7 & 51 & 5.13 & 15.8 & 36.6 & 2.33 & 9.83 & 26.6 & 1.11 \\
\hline & $\tilde{\tau}_{1,2 \mid \mathbf{Z}==}$ & 2.51 & 138 & 20.1 & 0.897 & 50.9 & 2.89 & 1.16 & 36.7 & 1.56 & 1.48 & 26.7 & 0.847 \\
\hline \multirow{4}{*}{ 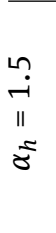 } & $\hat{\tau}_{1,2 \mid \mathbf{Z}=}^{(1)}$ & -59.1 & 104 & 28.1 & -14.7 & 42.3 & 3.87 & -7.56 & 29.7 & 1.86 & -4.17 & 21.8 & 0.932 \\
\hline & $\hat{\tau}_{1,2 \mid \mathbf{Z}=\cdot}^{(2)}$ & 4.34 & 99.7 & 21.4 & 2.05 & 42.1 & 3.48 & 2.07 & 29.6 & 1.75 & 1.35 & 21.8 & 0.899 \\
\hline & $\hat{\tau}_{1,2 \mid \mathbf{Z}=}^{(3)}$ & 67.8 & 103 & 29.6 & 18.8 & 42.3 & 3.96 & 11.7 & 29.6 & 1.92 & 6.87 & 21.8 & 0.957 \\
\hline & $\tilde{\boldsymbol{\tau}}_{1,2 \mid \mathbf{Z}=\cdot}$ & 3.34 & 103 & 13.4 & 2.08 & 42.5 & 2.6 & 2.08 & 29.7 & 1.39 & 1.35 & 21.8 & 0.755 \\
\hline \multirow{4}{*}{ 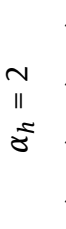 } & $\hat{\tau}_{1,2 \mid \mathbf{Z}=}^{(1)}$ & -37.2 & 88.2 & 23.9 & -9.57 & 38.2 & 4.6 & -3.75 & 26.2 & 2.34 & -1.09 & 19.8 & 1.32 \\
\hline & $\hat{\tau}_{1,2 \mid \mathbf{Z}=\cdot}^{(2)}$ & 8.17 & 85.9 & 21.2 & 2.69 & 38 & 4.45 & 3.32 & 26.1 & 2.3 & 2.99 & 19.8 & 1.32 \\
\hline & $\hat{\tau}_{1,2 \mid \mathbf{Z}=}^{(3)}$ & 53.5 & 87.4 & 25.3 & 14.9 & 38.1 & 4.74 & 10.4 & 26.2 & 2.41 & 7.08 & 19.8 & 1.36 \\
\hline & $\tilde{\boldsymbol{\tau}}_{1,2 \mid \mathbf{Z}}=$ & 8.47 & 88.5 & 15 & 2.69 & 38.4 & 3.59 & 3.33 & 26.3 & 1.93 & 3 & 19.9 & 1.15 \\
\hline
\end{tabular}

articles have studied the dependence between these two variables, see for instance [4, 29, 32]. Generally, in winter, electricity consumption increases when temperature decreases, because of the demand for heating. On the contrary, high temperatures in summer would cause an increased electricity demand for cooling homes, offices and so on.

Formally, we study the dependence between the following two variables:

- Power $_{t}$, the French electricity consumption ${ }^{4}$ in MW at time $t$;

- $\mathrm{Temp}_{t}$, the temperature in Celsius degree at the Orly Airport weather station (France) ${ }^{5}$.

These two variables are observed every 30 minutes from 01/01/1996 to 31/03/2019. The final dataset has got $n=329,756$ rows. The unconditional Kendall's tau between these two variables is -0.397 , computed using the fast Kendall's tau algorithm [14]. In other words, on average, lower temperatures are associated to higher electricity consumption.

4 downloaded from http://clients.rte-france.com/lang/an/visiteurs/vie/vie_stats_conso_inst.jsp

5 downloaded from https://gis.ncdc.noaa.gov/maps/ncei/cdo/hourly 
Table 3: Results of the simulation in Setting 2 using $h=\alpha_{h} \times h^{C V}$ where $h^{C V}$ has been chosen by cross-validation. All values have been multiplied by 1000 . Bold values indicate optimal choices for the chosen measure of performance.

\begin{tabular}{|c|c|c|c|c|c|c|c|c|c|c|c|c|c|}
\hline \multirow{6}{*}{$\begin{array}{l}\tilde{0} \\
0 \\
11 \\
\tilde{8}\end{array}$} & & \multicolumn{3}{|c|}{$n=100$} & \multicolumn{3}{|c|}{$n=500$} & \multicolumn{3}{|c|}{$n=1000$} & \multicolumn{3}{|c|}{$n=2000$} \\
\hline & \multirow{5}{*}{$\begin{array}{l}\hat{\tau}_{1,2 \mid \mathbf{Z}=}^{(1)} \\
\hat{\tau}_{1,2 \mid \mathbf{Z}=}^{(2)} \\
\hat{\tau}_{1,2 \mid \mathbf{Z}=}^{(3)} \\
\tilde{\tau}_{1,2 \mid \mathbf{Z}=}\end{array}$} & IBias & ISd & IMSE & IBias & ISd & IMSE & IBias & ISd & IMSE & IBias & ISd & IMSE \\
\hline & & -111 & 154 & 66.2 & -36.9 & 66.8 & 9.01 & -22.4 & 48.2 & 4.06 & -12.9 & 36.1 & 2.04 \\
\hline & & 0.0488 & 137 & 36.3 & 0.236 & 64.2 & 6.45 & 0.546 & 46.8 & 3.14 & 1.29 & 35.7 & 1.78 \\
\hline & & 111 & 151 & 60.6 & 37.4 & 66.3 & 8.88 & 23.5 & 47.2 & 4.07 & 15.5 & 36.2 & 2.18 \\
\hline & & 1.38 & 132 & 18.3 & 0.27 & 64.5 & 4.49 & 0.61 & 46.8 & 2.36 & 1.29 & 35.6 & 1.49 \\
\hline \multirow{4}{*}{ 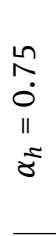 } & $\hat{\tau}_{1,2 \mid \mathrm{Z}=}^{(1)}$ & -67.4 & 117 & 35.7 & -23.3 & 52.1 & 5.27 & -13.9 & 37.8 & 2.4 & -7.6 & 29 & 1.3 \\
\hline & $\hat{\tau}_{1,2 \mid \mathbf{Z}=}^{(2)}$. & 4.32 & 108 & 23.5 & 0.809 & 50.7 & 4.21 & 1.03 & 37.2 & 2.07 & 1.78 & 28.8 & 1.21 \\
\hline & $\hat{\tau}_{1,2 \mid \mathbf{Z}=\cdot}^{(3)}$ & 76.1 & 119 & 35.4 & 24.9 & 51.6 & 5.12 & 16 & 37.6 & 2.49 & 11.2 & 29.1 & 1.39 \\
\hline & $\tilde{\tau}_{1,2 \mid \mathbf{Z}=}$ & 4.98 & 106 & 13.3 & 0.86 & 51.6 & 3.13 & 1.03 & 37.5 & 1.63 & 1.81 & 28.9 & 1.02 \\
\hline \multirow{4}{*}{$\begin{array}{l}\overparen{1} \\
\tilde{8}\end{array}$} & $\hat{\tau}_{1,2 \mid \mathbf{Z}=.}^{(1)}$ & -43 & 101 & 28 & -15.8 & 45.7 & 4.44 & -9.51 & 33.1 & 2.04 & -4.68 & 25.1 & 1.07 \\
\hline & $\hat{\tau}_{1,2 \mid \mathbf{Z}=}^{(2)}$. & 7.87 & 93.1 & 22.4 & 2.01 & 44.8 & 3.91 & 1.57 & 32.7 & 1.87 & 2.29 & 24.9 & 1.03 \\
\hline & $\hat{\tau}_{1,2 \mid \mathbf{Z}=.}^{(3)}$ & 58.8 & 97.6 & 27.2 & 19.8 & 45.3 & 4.41 & 12.7 & 32.9 & 2.1 & 9.27 & 25.1 & 1.14 \\
\hline & $\tilde{\tau}_{1,2 \mid \mathbf{Z}=.}$ & 8.51 & 98 & 15.7 & 2.05 & 46 & 3.01 & 1.57 & 33.1 & 1.5 & 2.33 & 25.1 & 0.871 \\
\hline \multirow{4}{*}{ 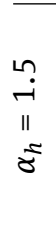 } & $\hat{\tau}_{1,2 \mid \mathbf{Z}=\cdot}^{(1)}$ & -16.1 & 95.6 & 41.7 & -6.36 & 43 & 6.35 & -4.04 & 30.6 & 2.87 & -1.11 & 22.1 & 1.34 \\
\hline & $\hat{\tau}_{1,2 \mid \mathbf{Z}=.}^{(2)}$ & 14.9 & 92.6 & 40.4 & 5.08 & 42.6 & 6.2 & 3.17 & 30.4 & 2.83 & 3.47 & 22 & 1.34 \\
\hline & $\hat{\tau}_{1,2 \mid \mathbf{Z}=\cdot}^{(3)}$ & 46 & 92.8 & 42.2 & 16.5 & 42.6 & 6.45 & 10.4 & 30.4 & 2.94 & 8.06 & 22.1 & 1.4 \\
\hline & $\tilde{\boldsymbol{\tau}}_{1,2 \mid \mathbf{Z}=.}$ & 15.6 & 100 & 35.2 & 5.11 & 44 & 5.31 & 3.17 & 31 & 2.45 & 3.5 & 22.4 & 1.17 \\
\hline
\end{tabular}

Table 4: Expectation and standard deviation of the bandwidth selected by cross-validation as a function of the sample size $n$, and comparison with bandwidth $h^{r e f}$ chosen by the rule-of-thumb.

\begin{tabular}{c|cccc}
\hline$n$ & 100 & 500 & 1000 & 2000 \\
\hline $\mathbb{E}\left[h^{C V}\right]$ & 0.77 & 0.43 & 0.34 & 0.27 \\
$S d\left[h^{C V}\right]$ & 0.17 & 0.091 & 0.060 & 0.057 \\
$h^{r e f}=n^{-1 / 5}$ & 0.40 & 0.29 & 0.25 & 0.22 \\
\hline
\end{tabular}

To have a more precise investigation about the dependence between these two variables, we decided to use a usual "detrending method": we fit a linear trend on both variables and consider only the dependence between the two series of residuals. Formally, our model assumption is

$$
\begin{array}{r}
\text { Power }_{t}=a_{0, \text { power }}+a_{1, \text { power }} \times t+\varepsilon_{1, t}, \\
\text { Temp }_{t}=a_{0, \text { temp }}+a_{1, \text { temp }} \times t+\varepsilon_{2, t},
\end{array}
$$

where $t$ is the the number of half-hours since $01 / 01 / 1996$, for some unknown coefficients $a_{0, \text { power }}, a_{1, \text { power }}$, $a_{0, \text { temp }}, a_{1, \text { temp }}$. And the couple of series $\left(\varepsilon_{1, t}, \varepsilon_{2, t}\right)$ is assumed to be stationary. We estimate these two linear regressions separately using ordinary least squares (OLS). The results are reported in Table 5. All the coefficients are significant. Indeed, because of economic and technological growth, the electricity consumption increases on average by $0.0044 \mathrm{MW}$ each hour. At the same time, temperature increases on average by $1.6 \times 10^{-6}$ Celsius degree per hour, which corresponds to a Global Warming of 0.014 degree per year. Even if this is a very simple model, with a linear growth, it correspond to the right order of magnitude commonly found. 
Table 5: Statistics for the OLS estimators of (5) and (6).

\begin{tabular}{lcccc}
\hline & Estimate & Std. Error & t-value & $\mathrm{p}$-value \\
\hline$a_{0, \text { power }}$ & $4.976 \mathrm{e}+04$ & $3.933 \mathrm{e}+01$ & 1265.1 & $<2 \mathrm{e}-16$ \\
$a_{1, \text { power }}$ & $2.205 \mathrm{e}-02$ & $2.066 \mathrm{e}-04$ & 106.8 & $<2 \mathrm{e}-16$ \\
\hline$a_{0, \text { temp }}$ & $1.187 \mathrm{e}+01$ & $2.526 \mathrm{e}-02$ & 469.902 & $<2 \mathrm{e}-16$ \\
$a_{1, \text { temp }}$ & $8.014 \mathrm{e}-07$ & $1.327 \mathrm{e}-07$ & 6.041 & $1.54 \mathrm{e}-09$ \\
\hline
\end{tabular}

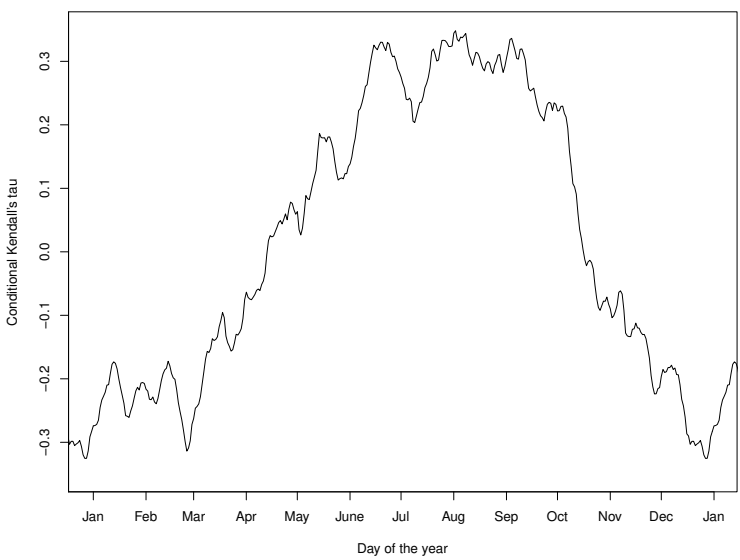

Figure 5: Conditional Kendall's tau between the detrended electricity consumption $\hat{\varepsilon}_{1, t}$ and the detrended temperature $\hat{\varepsilon}_{2, t}$, given the day of the year and estimated using $h_{C V}=5$ days.

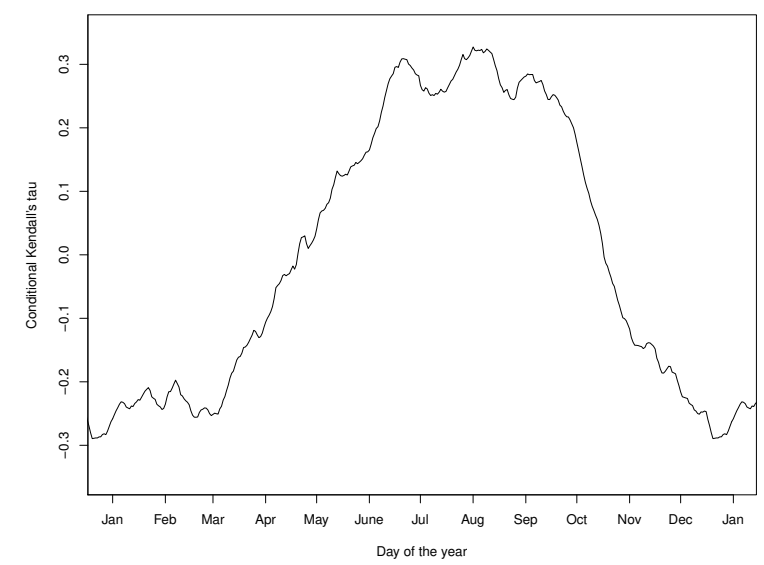

Figure 6: Conditional Kendall's tau between the detrended electricity consumption $\hat{\varepsilon}_{1, t}$ and the detrended temperature $\hat{\varepsilon}_{2, t}$, given the day of the year and estimated using $h_{\star}=12$ days.

Our goal is to estimate whether the dependence between electricity consumption and temperature is varying as a function of the day of the year (month, season...). The lack of stationarity on the original series had an influence on the conditional Kendall's tau. Indeed, a part of positive dependence between the original variables is due to the fact that they both increase on average over time. We consider this as a spurious effect caused by the non-stationarity. For this reason, we have studied the (conditional) dependence between the estimated residuals $\hat{\varepsilon}_{1, t}$ and $\hat{\varepsilon}_{2, t}$.

Concerning the bandwidth $h$ choice, we followed the insights of our simulation in Section 4. Globally, there exist two possibilities: choosing the bandwidth according to the usual rule-of-thumb $h_{\star}=1.5 \times \hat{\sigma}(Z) \times$ $n^{-1 / 5}$, or using our cross-validation criterion, which yields $h_{C V}$. Note that the computation time of this crossvalidation function is of order $O\left(n^{2}\right)$, by Equation (4). With our sample size $n=329756$, this criterion becomes computationally unfeasible in a reasonable time. To cope with this difficulty, we use a Monte-Carlo approximation

$$
C V_{N, \tilde{h}}(h):=\frac{1}{N} \sum_{l=1}^{N}\left(g_{k}\left(\mathbf{X}_{i_{l}}, \mathbf{X}_{j_{l}}\right)-\hat{\tau}_{-\left(i_{l}, j_{l}\right), 1,2 \mid \mathbf{Z}=\left(\mathbf{Z}_{i_{l}}+\mathbf{Z}_{j_{l}}\right) / 2}\right)^{2} \mathbb{1}\left(d\left(\mathbf{Z}_{i_{l}}, \mathbf{Z}_{j_{l}}\right) \leq \tilde{h}\right)
$$

where, for every $l=1, \ldots, N$, we sample independently $i_{l}$ uniformly in $[1, n]$ and $j_{l} \mid i_{l}$ uniformly on the set $\left\{j \in[1, n]: d\left(Z_{i_{l}}, Z_{j}\right) \leq \tilde{h}\right\}$. In practice, we choose $d(a, b)$ as the number of days between the two dates $a$ and $b$. For instance, the distance between January 1 st and December 30th is 2 days. Similarly, $\left(Z_{i_{l}}+Z_{j_{l}}\right) / 2$ corresponds to the mean day of the year between the days $Z_{i_{l}}$ and $Z_{j_{l}}$, and is computed using the package circular [2].

The estimated conditional Kendall's tau with the bandwidth $h_{C V}$ or $h \star$ are displayed in Figures 5 and 6 . We observe that conditional Kendall's tau is negative in winter, meaning that lower temperatures are associated 
with higher electricity demands. This can be explained by the energy consumption for heating purpose. On the contrary, in summer, higher temperatures are associated with higher energy demand, because of the energy consumption induced by cooling devices. It is interesting to note that the average conditional Kendall's tau in winter $(-0.23)$ is slightly smaller in absolute value than in summer $(0.30)$.

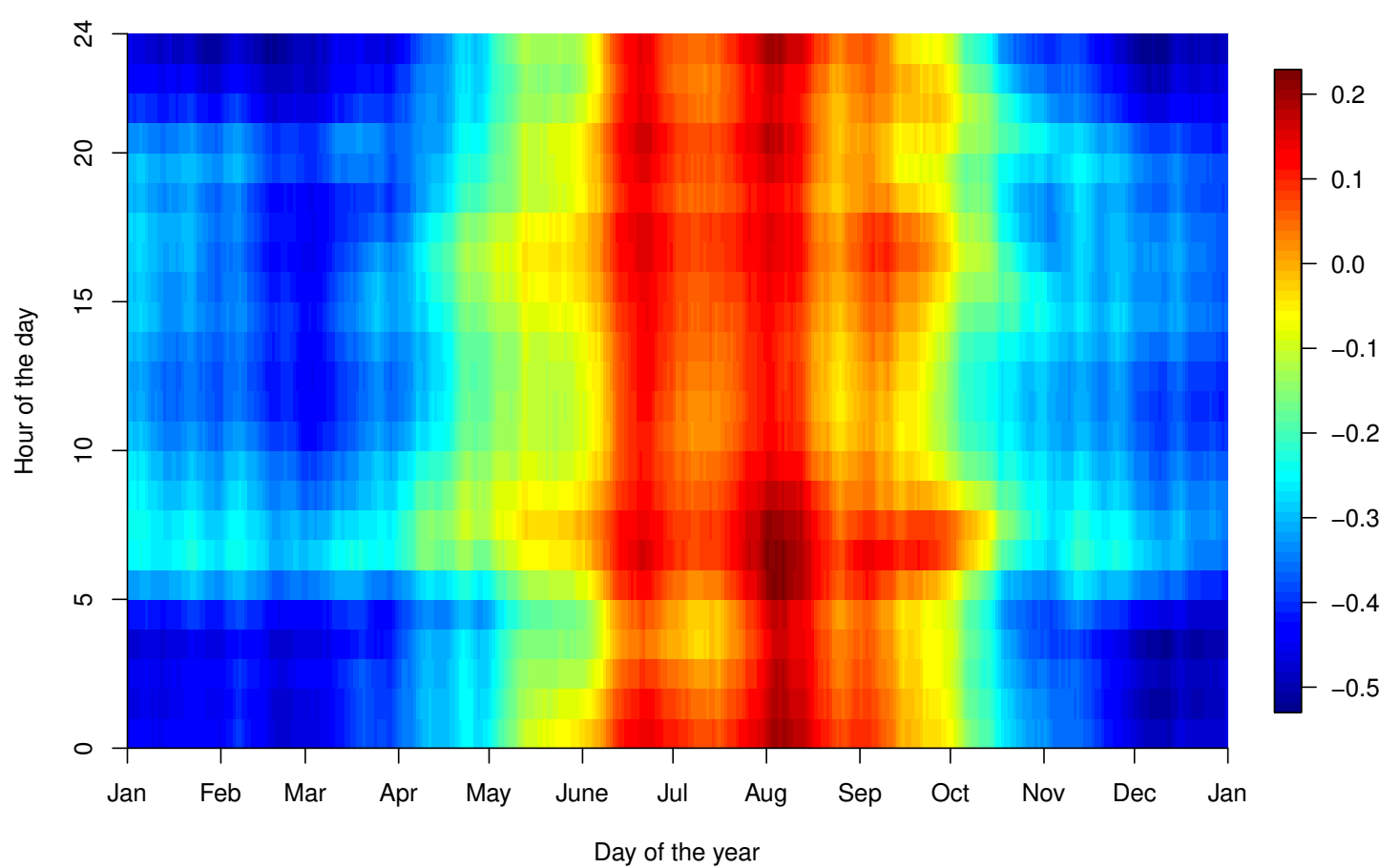

Figure 7: Conditional Kendall's tau between electricity consumption and temperature given the day of the year $\left(h_{*, 1}=12\right.$ days) and the time of the day (in hours, $h_{\star, 2}=1$ hour).

To complete this analysis, we decided to include a second variable, which is the hour of the day. The choice of a bivariate bandwidth is not straightforward. To simplify, we decide to use a diagonal bandwidth given by $h_{*, 1}=1.5 \times \hat{\sigma}\left(Z_{1}\right) \times n^{-1 / 5}=12$ days and $h_{*, 2}=1.5 \times \hat{\sigma}\left(Z_{2}\right) \times n^{-1 / 5}=1$ hour. The results are displayed on Figure 7. On the $x$-axis, we globally find the same trend: negative dependence in winter and positive dependence in summer, which is coherent. Moreover, in winter, the conditional Kendall's tau is more important (around -0.5) during nights (20:00-6:00) than in the daytime. This may be explained by the fact that heating in households has a more important contribution to the total consumption than during daytime, when many people live outside their homes.

Note that, during summers, the levels of Kendall's tau given date and daytime are most often smaller than Kendall's tau given date only. This may appear as counterintuitive. But, as noticed in [9], the average of the former quantity (over daytimes) is not equal to the latter quantity in general. In our particular case, we can argue that, during summers, the levels of dependence between temperature and energy consumption is rather weak once we control for daytime. This is the same phenomenon with usual factor models, where two variables may be independent given a third one, but they may be strongly dependent (unconditionnally).

\section{Acknowledgments}

This work is supported by the Labex Ecodec under the grant ANR-11-LABEX-0047 from the French Agence Nationale de la Recherche. 


\section{A Proofs}

For convenience, we recall Berk's (1970) inequality (see Theorem A in Serfling [37, p.201]). Note that, if $m=1$, this reduces to Bernstein's inequality.

Lemma 11. Let $m, n>0, \mathbf{X}_{1}, \ldots, \mathbf{X}_{n}$ i.i.d. random vectors with values in a measurable space $X$ and $g: X^{m} \rightarrow$ $[a, b]$ be a symmetric real bounded function. Set $\theta:=\mathbb{E}\left[g\left(\mathbf{X}_{1}, \ldots, \mathbf{X}_{m}\right)\right]$ and $\sigma^{2}:=\operatorname{Var}\left[g\left(\mathbf{X}_{1}, \ldots, \mathbf{X}_{m}\right)\right]$. Then, for any $t>0$ and $n \geq m$,

$$
\mathbb{P}\left(\left(\begin{array}{c}
n \\
m
\end{array}\right)^{-1} \sum_{c} g\left(\mathbf{X}_{i_{1}}, \ldots, \mathbf{X}_{i_{m}}\right)-\theta \geq t\right) \leq \exp \left(-\frac{[n / m] t^{2}}{2 \sigma^{2}+(2 / 3)(b-\theta) t}\right),
$$

where $\sum_{c}$ denotes summation over all subgroups of $m$ distinct integers $\left(i_{1}, \ldots, i_{m}\right)$ of $\{1, \ldots n\}$.

\section{A.1 Notations}

Let us define a few notations that will be used throughout the proofs. For every $1 \leq i, j \leq n$ and $\mathbf{z} \in \mathbb{R}^{p}$, let us define

$$
\begin{aligned}
& S_{i, j}(\mathbf{z}):=n^{-2} K_{h}\left(\mathbf{Z}_{i}-\mathbf{z}\right) K_{h}\left(\mathbf{Z}_{j}-\mathbf{z}\right)\left(\mathbb{1}\left\{\mathbf{X}_{i}<\mathbf{X}_{j}\right\}-\mathbb{P}\left(\mathbf{X}_{1}<\mathbf{X}_{2} \mid \mathbf{Z}_{1}=\mathbf{Z}_{2}=\mathbf{z}\right)\right), \\
& g_{\mathbf{z}}\left(\left(\mathbf{X}_{i}, \mathbf{Z}_{i}\right),\left(\mathbf{X}_{j}, \mathbf{Z}_{j}\right)\right):=K_{h}\left(\mathbf{Z}_{i}-\mathbf{z}\right) K_{h}\left(\mathbf{Z}_{j}-\mathbf{z}\right)\left(\mathbb{1}\left\{\mathbf{X}_{i}<\mathbf{X}_{j}\right\}-\mathbb{P}\left(\mathbf{X}_{i}<\mathbf{X}_{j} \mid \mathbf{Z}_{i}=\mathbf{Z}_{j}=\mathbf{z}\right)\right) \\
& -\mathbb{E}\left[K_{h}\left(\mathbf{Z}_{i}-\mathbf{z}\right) K_{h}\left(\mathbf{Z}_{j}-\mathbf{z}\right)\left(\mathbb{1}\left\{\mathbf{X}_{i}<\mathbf{X}_{j}\right\}-\mathbb{P}\left(\mathbf{X}_{i}<\mathbf{X}_{j} \mid \mathbf{Z}_{i}=\mathbf{Z}_{j}=\mathbf{z}\right)\right)\right], \\
& \tilde{g}_{i, j}=\left(g_{\mathbf{z}}\left(\left(\mathbf{X}_{i}, \mathbf{Z}_{i}\right),\left(\mathbf{X}_{j}, \mathbf{Z}_{j}\right)\right)+g_{\mathbf{z}}\left(\left(\mathbf{X}_{j}, \mathbf{Z}_{j}\right),\left(\mathbf{X}_{i}, \mathbf{Z}_{i}\right)\right)\right) / 2 \text {, } \\
& \bar{g}_{i}:=\mathbb{E}\left[\tilde{g}_{i, j} \mid \mathbf{X}_{i}, \mathbf{Z}_{i}\right] \text {, } \\
& \xi_{\mathbf{z}}\left(\mathbf{X}_{i}, \mathbf{Z}_{i}, \mathbf{X}_{j}, \mathbf{Z}_{j}\right):=\xi_{i, j}:=\tilde{g}_{i, j}-\bar{g}_{i}-\bar{g}_{j} \text {, } \\
& \ell_{\mathbf{z}}:\left(\mathbf{x}_{1}, \mathbf{z}_{1}, \mathbf{x}_{2}, \mathbf{z}_{2}\right) \mapsto \frac{h^{2 p}}{4 C_{K}^{2}} \xi_{\mathbf{z}}\left(\left(\mathbf{x}_{1}, \mathbf{z}_{1}\right),\left(\mathbf{x}_{2}, \mathbf{z}_{2}\right)\right) \text { for a given } h>0,
\end{aligned}
$$

Note that $\xi_{i, j}$ is a degenerate (symmetrical) U-statistics because $\mathbb{E}\left[\xi_{i, j} \mid \mathbf{X}_{i}, \mathbf{Z}_{i}\right]=\mathbb{E}\left[\xi_{i, j} \mid \mathbf{X}_{j}, \mathbf{Z}_{j}\right]=0$, when $i \neq j$.

In the proofs, we will study the difference $\hat{\tau}_{1,2 \mid \mathbf{Z}=\mathbf{z}}-\tau_{1,2 \mid \mathbf{Z}=\mathbf{z}}$ using two quantities that can be bounded separately: $\hat{f}_{\mathbf{Z}}^{2}(\mathbf{z})$ and $\sum_{1 \leq i, j \leq n} S_{i, j}(\mathbf{z})$.

$$
\begin{aligned}
\hat{\tau}_{1,2 \mid \mathbf{Z}=\mathbf{z}}-\tau_{1,2 \mid \mathbf{Z}=\mathbf{z}} & =4 \sum_{1 \leq i, j \leq n} w_{i, n}(\mathbf{z}) w_{j, n}(\mathbf{z}) \mathbb{1}\left\{\mathbf{X}_{i}<\mathbf{X}_{j}\right\}-4 \mathbb{P}\left(\mathbf{X}_{1}<\mathbf{X}_{2} \mid \mathbf{Z}_{1}=\mathbf{Z}_{2}=\mathbf{z}\right) \\
& =\frac{4}{n^{2} \hat{f}_{\mathbf{Z}}^{2}(\mathbf{z})} \sum_{1 \leq i, j \leq n} K_{h}\left(\mathbf{Z}_{i}-\mathbf{z}\right) K_{h}\left(\mathbf{Z}_{j}-\mathbf{z}\right)\left(\mathbb{1}\left\{\mathbf{X}_{i}<\mathbf{X}_{j}\right\}-\mathbb{P}\left(\mathbf{X}_{1}<\mathbf{X}_{2} \mid \mathbf{Z}_{1}=\mathbf{Z}_{2}=\mathbf{z}\right)\right) \\
& =\frac{4}{\hat{f}_{\mathbf{Z}}^{2}(\mathbf{z})} \sum_{1 \leq i, j \leq n} S_{i, j}(\mathbf{z}),
\end{aligned}
$$

This sum can be decomposed in the following way

$$
\sum_{1 \leq i, j \leq n} S_{i, j}(\mathbf{z})=\sum_{1 \leq i \neq j \leq n}\left(S_{i, j}(\mathbf{z})-\mathbb{E}\left[S_{i, j}(\mathbf{z})\right]\right)+n(n-1) \mathbb{E}\left[S_{1,2}(\mathbf{z})\right]-\Delta_{n}(\mathbf{z}) .
$$

where the "diagonal term" $\Delta_{n}(\mathbf{z}):=-\sum_{i=1}^{n} S_{i, i}(\mathbf{z})=\mathbb{P}\left(\mathbf{X}_{1}<\mathbf{X}_{2} \mid \mathbf{Z}_{1}=\mathbf{Z}_{2}=\mathbf{z}\right) \sum_{i=1}^{n} K_{h}^{2}\left(\mathbf{Z}_{i}-\mathbf{z}\right) / n^{2}$. The stochastic component above can itself be rewritten as

$$
\sum_{1 \leq i \neq j \leq n}\left(S_{i, j}(\mathbf{z})-\mathbb{E}\left[S_{i, j}(\mathbf{z})\right]\right)=\frac{1}{n^{2}} \sum_{1 \leq i \neq j \leq n} g_{\mathbf{z}}\left(\left(\mathbf{X}_{i}, \mathbf{Z}_{i}\right),\left(\mathbf{X}_{j}, \mathbf{Z}_{j}\right)\right)=\frac{1}{n^{2}} \sum_{1 \leq i \neq j \leq n} \tilde{g}_{i, j}
$$




$$
=\frac{1}{n^{2}} \sum_{1 \leq i \neq j \leq n} \xi_{i, j}+\frac{2(n-1)}{n^{2}} \sum_{i=1}^{n} \bar{g}_{i} .
$$

\section{A.2 Proof of Proposition 1}

Since there are no ties a.s.,

$$
\begin{aligned}
1+\hat{\tau}_{1,2 \mid \mathbf{Z}=\mathbf{z}}^{(1)} & =4 \sum_{i=1}^{n} \sum_{j=1}^{n} w_{i, n}(\mathbf{z}) w_{j, n}(\mathbf{z})\left(\mathbb{1}\left\{X_{i, 1}<X_{j, 1}\right\}-\mathbb{1}\left\{X_{i, 1}<X_{j, 1}, X_{i, 2}>X_{j, 2}\right\}\right) \\
& =4 \sum_{i=1}^{n} \sum_{j=1}^{n} w_{i, n}(\mathbf{z}) w_{j, n}(\mathbf{z}) \mathbb{1}\left\{X_{i, 1}<X_{j, 1}\right\}+\hat{\tau}_{1,2 \mid \mathbf{Z}=\mathbf{z}}^{(3)}-1 .
\end{aligned}
$$

But

$$
\begin{aligned}
1 & =\sum_{i=1}^{n} \sum_{j=1}^{n} w_{i, n}(\mathbf{z}) w_{j, n}(\mathbf{z})=\sum_{i=1}^{n} \sum_{j=1}^{n} w_{i, n}(\mathbf{z}) w_{j, n}(\mathbf{z})\left(\mathbb{1}\left\{X_{i, 1} \leq X_{j, 1}\right\}+\mathbb{1}\left\{X_{i, 1}>X_{j, 1}\right\}\right) \\
& =2 \sum_{i=1}^{n} \sum_{j=1}^{n} w_{i, n}(\mathbf{z}) w_{j, n}(\mathbf{z}) \mathbb{1}\left\{X_{i, 1}<X_{j, 1}\right\}+\sum_{i=1}^{n} w_{i, n}^{2}(\mathbf{z}),
\end{aligned}
$$

implying $1+\hat{\tau}_{1,2 \mid \mathbf{Z}=\mathbf{z}}^{(1)}=2\left(1-s_{n}\right)+\hat{\tau}_{1,2 \mid \mathbf{Z}=\mathbf{z}}^{(3)}-1$, and then $\hat{\tau}_{1,2 \mid \mathbf{Z}=\mathbf{z}}^{(1)}=\hat{\tau}_{1,2 \mid \mathbf{Z}=\mathbf{Z}}^{(3)}-2 s_{n}$. Moreover,

$$
\begin{aligned}
\hat{\tau}_{1,2 \mid \mathbf{Z}=\mathbf{z}}^{(2)} & =\sum_{i=1}^{n} \sum_{j=1}^{n} w_{i, n}(\mathbf{z}) w_{j, n}(\mathbf{z})\left(\mathbb{1}\left\{X_{i, 1}>X_{j, 1}, X_{i, 2}>X_{j, 2}\right\}+\mathbb{1}\left\{X_{i, 1}<X_{j, 1}, X_{i, 2}<X_{j, 2}\right\}\right. \\
& \left.-\mathbb{1}\left\{X_{i, 1}>X_{j, 1}, X_{i, 2}<X_{j, 2}\right\}-\mathbb{1}\left\{X_{i, 1}<X_{j, 1}, X_{i, 2}>X_{j, 2}\right\}\right) \\
& =2 \sum_{i=1}^{n} \sum_{j=1}^{n} w_{i, n}(\mathbf{z}) w_{j, n}(\mathbf{z})\left(\mathbb{1}\left\{X_{i, 1}>X_{j, 1}, X_{i, 2}>X_{j, 2}\right\}-\mathbb{1}\left\{X_{i, 1}>X_{j, 1}, X_{i, 2}<X_{j, 2}\right\}\right) \\
& =\frac{1}{2}\left(\hat{\tau}_{1,2 \mid \mathbf{Z}=\mathbf{z}}^{(1)}+1\right)+\frac{1}{2}\left(\hat{\tau}_{1,2 \mid \mathbf{Z}=\mathbf{z}}^{(3)}-1\right)=\frac{\hat{\tau}_{1,2 \mid \mathbf{Z}=\mathbf{z}}^{(1)}+\hat{\tau}_{1,2 \mid \mathbf{Z}=\mathbf{z}}^{(3)}=\hat{\tau}_{1,2 \mid \mathbf{Z}=\mathbf{z}}^{(1)}+s_{n}=\hat{\tau}_{1,2 \mid \mathbf{Z}=\mathbf{z}}^{(3)}-s_{n} .}{2}
\end{aligned}
$$

\section{A.3 Proof of Proposition 2}

Lemma 12. Under Assumptions 3.1, 3.2 and 3.3, we have for any $t>0$,

$$
\mathbb{P}\left(\left|\hat{f}_{\mathbf{Z}}(\mathbf{z})-f_{\mathbf{Z}}(\mathbf{z})\right| \geq \frac{C_{K, \alpha} h^{\alpha}}{\alpha !}+t\right) \leq 2 \exp \left(-\frac{n h^{p} t^{2}}{2 f_{\mathbf{Z}, \max } \int K^{2}+(2 / 3) C_{K} t}\right) .
$$

This Lemma is proved below. If, for some $\epsilon>0$, we have $C_{K, \alpha} h^{\alpha} / \alpha !+t \leq f_{\mathbf{Z}, \min }-\epsilon$, then $\hat{f}(\mathbf{z}) \geq \epsilon>0$ with a probability larger than $1-2 \exp \left(-n h^{p} t^{2} /\left(2 f_{\mathbf{Z} \text {, max }} \int K^{2}+(2 / 3) C_{K} t\right)\right)$. So, we should choose the largest $t$ as possible, which yields Proposition 2.

It remains to prove Lemma 12. Use the usual decomposition between a stochastic component and a bias: $\hat{f}_{\mathbf{Z}}(\mathbf{z})-f_{\mathbf{Z}}(\mathbf{z})=\left(\hat{f}_{\mathbf{Z}}(\mathbf{z})-\mathbb{E}\left[\hat{f}_{\mathbf{Z}}(\mathbf{z})\right]\right)+\left(\mathbb{E}\left[\hat{f}_{\mathbf{Z}}(\mathbf{z})\right]-f_{\mathbf{Z}}(\mathbf{z})\right)$. We first bound the bias from above.

$$
\mathbb{E}\left[\hat{f}_{\mathbf{Z}}(\mathbf{z})\right]-f_{\mathbf{Z}}(\mathbf{z})=\int_{\mathbb{R}^{p}} K(\mathbf{u})\left(f_{\mathbf{Z}}(\mathbf{z}+h \mathbf{u})-f_{\mathbf{Z}}(\mathbf{z})\right) d \mathbf{u} .
$$


Set $\phi_{\mathbf{z}, \mathbf{u}}(t):=f_{\mathbf{Z}}(\mathbf{z}+t h \mathbf{u})$ for $t \in[0,1]$. This function has at least the same regularity as $f_{\mathbf{Z}}$, so it is $\alpha$ differentiable (by Assumption 3.2). By a Taylor-Lagrange expansion, we get

$$
\int_{\mathbb{R}^{p}} K(\mathbf{u})\left(f_{\mathbf{Z}}(\mathbf{z}+h \mathbf{u})-f_{\mathbf{Z}}(\mathbf{z})\right) d \mathbf{u}=\int_{\mathbb{R}^{p}} K(\mathbf{u})\left(\sum_{i=1}^{\alpha-1} \frac{1}{i !} \phi_{\mathbf{z}, \mathbf{u}}^{(i)}(0)+\frac{1}{\alpha !} \phi_{\mathbf{z}, \mathbf{u}}^{(\alpha)}\left(t_{\mathbf{z}, \mathbf{u}}\right)\right) d \mathbf{u},
$$

for some real number $t_{\mathbf{z}, \mathbf{u}} \in(0,1)$. By Assumption 3.1(c) and for every $i<\alpha, \int_{\mathbb{R}^{p}} K(\mathbf{u}) \phi_{\mathbf{z}, \mathbf{u}}^{(i)}(0) d \mathbf{u}=0$. Therefore,

$$
\begin{aligned}
& \left|\mathbb{E}\left[\hat{f}_{\mathbf{Z}}(\mathbf{z})\right]-f_{\mathbf{Z}}(\mathbf{z})\right|=\left|\int_{\mathbb{R}^{p}} K(\mathbf{u}) \frac{1}{\alpha !} \phi_{\mathbf{z}, \mathbf{u}}^{(\alpha)}\left(t_{\mathbf{z}, \mathbf{u}}\right) d \mathbf{u}\right| \\
& \quad=\frac{1}{\alpha !}\left|\int_{\mathbb{R}^{p}} K(\mathbf{u}) \sum_{i_{1}, \ldots, i_{\alpha}=1}^{p} h^{\alpha} u_{i_{1}} \ldots u_{i_{\alpha}} \frac{\partial^{\alpha} f_{\mathbf{Z}}}{\partial z_{i_{1}} \ldots \partial z_{i_{\alpha}}}\left(\mathbf{z}+t_{\mathbf{z}, \mathbf{u}} h \mathbf{u}\right) d \mathbf{u}\right| \leq \frac{C_{K, \alpha}}{\alpha !} h^{\alpha},
\end{aligned}
$$

where the last inequality results from Assumption 3.2.

Second, the stochastic component may be written as

$$
\hat{f}_{\mathbf{Z}}(\mathbf{z})-\mathbb{E}\left[\hat{f}_{\mathbf{Z}}(\mathbf{z})\right]=n^{-1} \sum_{i=1}^{n} K_{h}\left(\mathbf{Z}_{i}-\mathbf{z}\right)-\mathbb{E}\left[n^{-1} \sum_{i=1}^{n} K_{h}\left(\mathbf{Z}_{i}-\mathbf{z}\right)\right]=n^{-1} \sum_{i=1}^{n}\left(g\left(\mathbf{Z}_{i}\right)-\mathbb{E}\left[g\left(\mathbf{Z}_{i}\right)\right]\right),
$$

where $g\left(\mathbf{Z}_{i}\right):=K_{h}\left(\mathbf{Z}_{i}-\mathbf{z}\right)$. Apply Lemma 11 with $m=1$ and the latter $g\left(\mathbf{Z}_{i}\right)$. Here, we have $b=-a=h^{-p} C_{K}$ (by Assumption 3.1(a)), $\theta=\mathbb{E}\left[g\left(\mathbf{Z}_{1}\right)\right] \geq 0$ (by Assumption 3.1(d)), and $\left|\operatorname{Var}\left[g\left(\mathbf{Z}_{1}\right)\right]\right| \leq h^{-p} f_{\mathbf{Z}, \max } \int K^{2}$ (combining Assumptions 3.1(b) and 3.3), so that we get

$$
\mathbb{P}\left(\left|\frac{1}{n} \sum_{i=1}^{n} K_{h}\left(\mathbf{Z}_{i}-\mathbf{z}\right)-\mathbb{E}\left[K_{h}\left(\mathbf{Z}_{i}-\mathbf{z}\right)\right]\right| \geq t\right) \leq 2 \exp \left(-\frac{n t^{2}}{2 h^{-p} f_{\mathbf{Z}, \text { max }} \int K^{2}+(2 / 3) h^{-p} C_{K} t}\right) .
$$

\section{A.4 Proof of Proposition 3}

We show the result for $k=1$. The two other cases can be proven in the same way. Using the decomposition (14), for any positive numbers $x$ and $\lambda(\mathbf{z})$, we have

$$
\begin{aligned}
& \mathbb{P}\left(\left|\hat{\tau}_{1,2 \mid \mathbf{Z}=\mathbf{z}}-\tau_{1,2 \mid \mathbf{Z}=\mathbf{z}}\right|>x\right) \leq \mathbb{P}\left(\frac{1}{\hat{f}_{\mathbf{Z}}^{2}(\mathbf{z})}>\frac{1+\lambda(\mathbf{z})}{f_{\mathbf{Z}}^{2}(\mathbf{z})}\right)+\mathbb{P}\left(\frac{4(1+\lambda(\mathbf{z}))}{f_{\mathbf{Z}}^{2}(\mathbf{z})} \times\left|\sum_{1 \leq i, j \leq n} S_{i, j}(\mathbf{z})\right|>x\right) \\
& \quad \leq \mathbb{P}\left(\left|\frac{1}{\hat{f}_{\mathbf{Z}}^{2}(\mathbf{z})}-\frac{1}{f_{\mathbf{Z}}^{2}(\mathbf{z})}\right|>\frac{\lambda(\mathbf{z})}{f_{\mathbf{Z}}^{2}(\mathbf{z})}\right)+\mathbb{P}\left(\frac{4(1+\lambda(\mathbf{z}))}{f_{\mathbf{Z}}^{2}(\mathbf{z})} \times\left|\sum_{1 \leq i, j \leq n} S_{i, j}(\mathbf{z})\right|>x\right) .
\end{aligned}
$$

For any $t$ s.t. $C_{K, \alpha} h^{\alpha} / \alpha !+t<f_{\mathbf{Z}, \min } / 2$, set $\lambda(\mathbf{z})=16 f_{\mathbf{Z}}^{2}(\mathbf{z})\left(C_{K, \alpha} h^{\alpha} / \alpha !+t\right) / f_{\mathbf{Z}, \min }^{3}$. This yields

$$
\begin{aligned}
\mathbb{P}\left(\left|\hat{\tau}_{1,2 \mid \mathbf{Z}=\mathbf{z}}-\tau_{1,2 \mid \mathbf{Z}=\mathbf{z}}\right|>x\right) & \leq \mathbb{P}\left(\left|\frac{1}{\hat{f}_{\mathbf{Z}}^{2}(\mathbf{z})}-\frac{1}{f_{\mathbf{Z}}^{2}(\mathbf{z})}\right|>\frac{16}{f_{\mathbf{Z}, \min }^{3}}\left(\frac{C_{K, \alpha} h^{\alpha}}{\alpha !}+t\right)\right) \\
& +\mathbb{P}\left(\left|\sum_{1 \leq i, j \leq n} S_{i, j}(\mathbf{z})\right|>\frac{f_{\mathbf{Z}}^{2}(\mathbf{z}) x}{4(1+\lambda(\mathbf{z}))}\right) .
\end{aligned}
$$

By setting

$$
x=\frac{4}{f_{\mathbf{Z}}^{2}(\mathbf{z})}\left(\frac{C_{\mathbf{X Z}, \alpha} h^{\alpha}}{\alpha !}+\frac{3 f_{\mathbf{z}}(\mathbf{z}) \int K^{2}}{2 n h^{p}}+t^{\prime}\right)\left(1+\frac{16 f_{\mathbf{Z}}^{2}(\mathbf{z})}{f_{\mathbf{Z}, \min }^{3}}\left(\frac{C_{K, \alpha} h^{\alpha}}{\alpha !}+t\right)\right),
$$

and applying the next two lemmas 13 and 14, we get the result. 
Lemma 13. Under Assumptions 3.1-3.3 and if $C_{K, \alpha} h^{\alpha} / \alpha !+t<f_{\mathrm{Z}, \min } / 2$ for some $t>0$,

$$
\mathbb{P}\left(\left|\frac{1}{\hat{f}_{\mathbf{Z}}^{2}(\mathbf{z})}-\frac{1}{f_{\mathbf{Z}}^{2}(\mathbf{z})}\right|>\frac{16}{f_{\mathbf{Z}, \min }^{3}}\left(\frac{C_{K, \alpha} h^{\alpha}}{\alpha !}+t\right)\right) \leq 2 \exp \left(-\frac{n h^{p} t^{2}}{2 f_{\mathbf{Z}, \max } \int K^{2}+(2 / 3) C_{K} t}\right),
$$

and $\hat{f}_{\mathbf{Z}}(\mathbf{z})$ is strictly positive on these events.

Proof: Applying the mean value inequality to the function $x \mapsto 1 / x^{2}$, we get the inequality $\left|1 / \hat{f}_{Z}^{2}(\mathbf{z})-1 / f_{Z}^{2}(\mathbf{z})\right| \leq$ $2\left|\hat{f}_{\mathbf{Z}}(\mathbf{z})-f_{\mathbf{Z}}(\mathbf{z})\right| / f_{\mathbf{Z}}^{\star 3}$, where $f_{\mathbf{Z}}^{\star}$ lies between $\hat{f}_{\mathbf{Z}}(\mathbf{z})$ and $f_{\mathbf{Z}}(\mathbf{z})$. Denote by $\mathcal{E}$ the event $\mathcal{E}:=\left\{\hat{f}_{\mathbf{Z}}(\mathbf{z})-f_{\mathbf{Z}}(\mathbf{z}) \mid \leq\right.$ $\left.C_{K, \alpha} h^{\alpha} / \alpha !+t\right\}$. By Lemma 12 , we obtain

$$
\mathbb{P}(\mathcal{E}) \geq 1-2 \exp \left(-\frac{n h^{p} t^{2}}{2 f_{\mathbf{Z}, \max } \int K^{2}+(2 / 3) C_{K} t}\right) .
$$

Therefore, on this event $\mathcal{E},\left|\hat{f}_{\mathbf{Z}}(\mathbf{z})-f_{\mathbf{Z}}(\mathbf{z})\right| \leq f_{\mathbf{Z}, \min } / 2$. Moreover, we have $f_{\mathbf{Z}, \min } \leq f_{\mathbf{Z}}(\mathbf{z})$ using Assumption 3.3 and then $f_{\mathrm{Z}, \min } / 2 \leq f_{\mathrm{Z}}^{\star}$. Combining the previous inequalities, we finally get

$$
\left|\frac{1}{\hat{f}_{\mathbf{Z}}^{2}(\mathbf{z})}-\frac{1}{f_{\mathbf{Z}}^{2}(\mathbf{z})}\right| \leq \frac{16}{f_{\mathbf{Z}, \text { min }}^{3}}\left|\hat{f}_{\mathbf{Z}}(\mathbf{z})-f_{\mathbf{Z}}(\mathbf{z})\right| \leq \frac{16}{f_{\mathbf{Z}, \text { min }}^{3}}\left(\frac{C_{K, \alpha} h^{\alpha}}{\alpha !}+t\right),
$$

on $\mathcal{E}$. But since

$$
\mathbb{P}\left(\left|\frac{1}{\hat{f}_{\mathbf{Z}}^{2}(\mathbf{z})}-\frac{1}{f_{\mathbf{Z}}^{2}(\mathbf{z})}\right|>\frac{16}{f_{\mathbf{Z}, \min }^{3}}\left(\frac{C_{K, \alpha} h^{\alpha}}{\alpha !}+t\right)\right) \leq \mathbb{P}\left(\mathcal{E}^{c}\right),
$$

we deduce the result.

Lemma 14. Under Assumptions 3.1-3.4, if $C_{\tilde{K}, 2} h^{2}<f_{\mathbf{z}}(\mathbf{z})$, we have for any $t>0$

$$
\begin{aligned}
& \mathbb{P}\left(\left|\sum_{1 \leq i, j \leq n} S_{i, j}(\mathbf{z})\right|>\frac{C_{\mathbf{X Z}, \alpha} h^{\alpha}}{\alpha !}+\frac{3 f_{\mathbf{z}}(\mathbf{z}) \int K^{2}}{2 n h^{p}}+t\right) \leq 2 \exp \left(-\frac{(n-1) h^{2 p} t^{2}}{4 f_{\mathbf{Z}, \max }^{2}\left(\int K^{2}\right)^{2}+(8 / 3) C_{K}^{2} t}\right) \\
& \quad+2 \exp \left(-\frac{n h^{p}\left(f_{\mathbf{z}}(\mathbf{z})-C_{\tilde{K}, 2} h^{2}\right)^{2}}{8 f_{\mathbf{Z}, \max } \int \tilde{K}^{2}+4 C_{\tilde{K}}\left(f_{\mathbf{z}}(\mathbf{z})-C_{\tilde{K}, 2} h^{2}\right) / 3}\right) .
\end{aligned}
$$

Proof: We will use the decomposition of $\sum_{1 \leq i, j \leq n} S_{i, j}(\mathbf{z})$ given in Equation (15) and bound separately each of its three components with high probability.

We first bound the negligible diagonal term $\Delta_{n}$. Note that $\tilde{K}(\cdot):=K^{2}(\cdot) / \int K^{2}$ is a two-order kernel, so that $\tilde{f}_{\mathbf{z}}(\mathbf{z}):=\sum_{i=1}^{n} \tilde{K}_{h}\left(\mathbf{Z}_{i}-\mathbf{z}\right) / n$ can be used as an estimator of $f_{\mathbf{Z}}(\mathbf{z})$, where $\tilde{K}_{h}(\cdot):=h^{-p} \tilde{K}(\cdot / h)$. Therefore, applying Lemma 12 to $\tilde{f}$, we have for $\varepsilon>0$ such that $C_{\tilde{K}, 2} h^{2} / 2+\varepsilon=f_{\mathbf{z}}(\mathbf{z}) / 2$,

$$
\begin{aligned}
\mathbb{P}\left(\Delta_{n} \geq \frac{3 f_{\mathbf{Z}}(\mathbf{z}) \int K^{2}}{2 n h^{p}}\right) & \leq \mathbb{P}\left(\left|\frac{\int K^{2}}{n^{2} h^{p}} \sum_{i=1}^{n} \tilde{K}_{h}\left(\mathbf{Z}_{i}-\mathbf{z}\right)-\frac{f_{\mathbf{Z}}(\mathbf{z}) \int K^{2}}{n h^{p}}\right| \geq\left(\frac{\int K^{2}}{n h^{p}}\right)\left(\frac{C_{\tilde{K}, 2} h^{2}}{2}+\varepsilon\right)\right) \\
& \leq \mathbb{P}\left(\left|\tilde{f}_{\mathbf{Z}}(\mathbf{z})-f_{\mathbf{Z}}(\mathbf{z})\right| \geq \frac{C_{\tilde{K}, 2} h^{2}}{2}+\varepsilon\right) \\
& \leq 2 \exp \left(-\frac{n h^{p} \varepsilon^{2}}{2 f_{\mathbf{Z}, \max } \int \tilde{K}^{2}+(2 / 3) C_{\tilde{K}} \varepsilon}\right) .
\end{aligned}
$$

Second, let us deal with the bias term. Simple calculations provide, if $i \neq j$,

$$
\mathbb{E}\left[S_{i, j}(\mathbf{z})\right]=n^{-2} \mathbb{E}\left[K_{h}\left(\mathbf{Z}_{i}-\mathbf{z}\right) K_{h}\left(\mathbf{Z}_{j}-\mathbf{z}\right)\left(\mathbb{1}\left\{\mathbf{X}_{i}<\mathbf{X}_{j}\right\}-\mathbb{P}\left(\mathbf{X}_{i}<\mathbf{X}_{j} \mid \mathbf{Z}_{i}=\mathbf{Z}_{j}=\mathbf{z}\right)\right)\right]
$$




$$
\begin{aligned}
& =n^{-2} \int_{\mathbb{R}^{2 p+2}} K_{h}\left(\mathbf{z}_{1}-\mathbf{z}\right) K_{h}\left(\mathbf{z}_{2}-\mathbf{z}\right)\left(\mathbb{1}\left\{\mathbf{x}_{1}<\mathbf{x}_{2}\right\}-\mathbb{P}\left(\mathbf{X}_{i}<\mathbf{X}_{j} \mid \mathbf{Z}_{i}=\mathbf{Z}_{j}=\mathbf{z}\right)\right) \\
& \quad \times f_{\mathbf{X}, \mathbf{Z}}\left(\mathbf{x}_{1}, \mathbf{z}_{1}\right) f_{\mathbf{X}, \mathbf{Z}}\left(\mathbf{x}_{2}, \mathbf{z}_{2}\right) d \mathbf{x}_{1} d \mathbf{z}_{1} d \mathbf{x}_{2} d \mathbf{z}_{2} \\
& =n^{-2} \int_{\mathbb{R}^{2 p+2}} K(\mathbf{u}) K(\mathbf{v})\left(\mathbb{1}\left\{\mathbf{x}_{1}<\mathbf{x}_{2}\right\}-\mathbb{P}\left(\mathbf{X}_{i}<\mathbf{X}_{j} \mid \mathbf{Z}_{i}=\mathbf{Z}_{j}=\mathbf{z}\right)\right) \\
& \quad \times\left(f_{\mathbf{X}, \mathbf{Z}}\left(\mathbf{x}_{1}, \mathbf{z}+h \mathbf{u}\right) f_{\mathbf{X}, \mathbf{Z}}\left(\mathbf{x}_{2}, \mathbf{z}+h \mathbf{v}\right)-f_{\mathbf{X}, \mathbf{Z}}\left(\mathbf{x}_{1}, \mathbf{z}\right) f_{\mathbf{X}, \mathbf{Z}}\left(\mathbf{x}_{2}, \mathbf{z}\right)\right) d \mathbf{x}_{1} d \mathbf{u} d \mathbf{x}_{2} d \mathbf{v},
\end{aligned}
$$

because, for every $\mathbf{z}$,

$$
0=\int_{\mathbb{R}^{4}}\left(\mathbb{1}\left\{\mathbf{x}_{1}<\mathbf{x}_{2}\right\}-\mathbb{P}\left(\mathbf{X}_{1}<\mathbf{X}_{2} \mid \mathbf{Z}_{1}=\mathbf{Z}_{2}=\mathbf{z}\right)\right) f_{\mathbf{X}, \mathbf{Z}}\left(\mathbf{x}_{1}, \mathbf{z}\right) f_{\mathbf{X}, \mathbf{Z}}\left(\mathbf{x}_{2}, \mathbf{z}\right) d \mathbf{x}_{1} d \mathbf{x}_{2} .
$$

Apply the Taylor-Lagrange formula to the function $\phi_{\mathbf{x}_{1}, \mathbf{x}_{2}, \mathbf{u}, \mathbf{v}}(t):=f_{\mathbf{X}, \mathbf{Z}}\left(\mathbf{x}_{1}, \mathbf{z}+t h \mathbf{u}\right) f_{\mathbf{X}, \mathbf{Z}}\left(\mathbf{x}_{2}, \mathbf{z}+\right.$ th $\left.\mathbf{v}\right)$ which is differentiable by Assumption 3.4. This yields

$$
\begin{aligned}
\mathbb{E}\left[S_{i, j}(\mathbf{z})\right]= & n^{-2} \int K(\mathbf{u}) K(\mathbf{v})\left(\mathbb{1}\left\{\mathbf{x}_{1}<\mathbf{x}_{2}\right\}-\mathbb{P}\left(\mathbf{X}_{i}<\mathbf{X}_{j} \mid \mathbf{Z}_{i}=\mathbf{Z}_{j}=\mathbf{z}\right)\right) \\
& \times\left(\sum_{k=1}^{\alpha-1} \frac{1}{k !} \phi_{\mathbf{x}_{1}, \mathbf{x}_{2}, \mathbf{u}, \mathbf{v}}^{(k)}(0)+\frac{1}{\alpha !} \phi_{\mathbf{x}_{1}, \mathbf{x}_{2}, \mathbf{u}, \mathbf{v}}^{(\alpha)}\left(t_{\mathbf{x}_{1}, \mathbf{x}_{2}, \mathbf{u}, \mathbf{v}}\right)\right) d \mathbf{x}_{1} d \mathbf{u} d \mathbf{x}_{2} d \mathbf{v} \\
= & \int \frac{K(\mathbf{u}) K(\mathbf{v})}{n^{2} \alpha !}\left(\mathbb{1}\left\{\mathbf{x}_{1}<\mathbf{x}_{2}\right\}-\mathbb{P}\left(\mathbf{X}_{i}<\mathbf{X}_{j} \mid \mathbf{Z}_{i}=\mathbf{Z}_{j}=\mathbf{z}\right)\right) \phi_{\mathbf{x}_{1}, \mathbf{x}_{2}, \mathbf{u}, \mathbf{v}}^{(\alpha)}\left(t_{\mathbf{x}_{1}, \mathbf{x}_{2}, \mathbf{u}, \mathbf{v}}\right) d \mathbf{x}_{1} d \mathbf{u} d \mathbf{x}_{2} d \mathbf{v} .
\end{aligned}
$$

Since $\phi_{\mathbf{x}_{1}, \mathbf{x}_{2}, \mathbf{u}, \mathbf{v}}^{(\alpha)}(t)$ is equal to

$$
\sum_{k=0}^{\alpha}\left(\begin{array}{l}
\alpha \\
k
\end{array}\right) \sum_{i_{1}, \ldots, i_{\alpha}=1}^{p} h^{\alpha} u_{i_{1}} \ldots u_{i_{k}} v_{i_{k+1}} \ldots v_{i_{\alpha}} \frac{\partial^{k} f_{\mathbf{X}, \mathbf{Z}}}{\partial z_{i_{1}} \ldots \partial z_{i_{k}}}\left(\mathbf{x}_{1}, \mathbf{z}+t h \mathbf{u}\right) \frac{\partial^{\alpha-k} f_{\mathbf{X}, \mathbf{Z}}}{\partial z_{i_{k+1}} \ldots \partial z_{i_{\alpha}}}\left(\mathbf{x}_{2}, \mathbf{z}+\text { th } \mathbf{v}\right),
$$

using Assumption 3.4, we get

$$
\left|\mathbb{E}\left[S_{1,2}(\mathbf{z})\right]\right| \leq C_{\mathbf{X Z}, \alpha} h^{\alpha} /\left(n^{2} \alpha !\right) .
$$

Third, the stochastic component will be bounded from above. Indeed,

$$
\sum_{1 \leq i \neq j \leq n}\left(S_{i, j}(\mathbf{z})-\mathbb{E}\left[S_{i, j}(\mathbf{z})\right]\right)=\frac{1}{n^{2}} \sum_{1 \leq i \neq j \leq n} g_{\mathbf{z}}\left(\left(\mathbf{X}_{i}, \mathbf{Z}_{i}\right),\left(\mathbf{X}_{j}, \mathbf{Z}_{j}\right)\right),
$$

with the function $g_{\mathbf{z}}$ defined in (9). We can now apply Lemma 11 to the sum of the $\tilde{g}_{i, j}$, which are symmetrized versions of $g_{\mathbf{z}}$. With this notation, $\theta=\mathbb{E}\left[\tilde{g}_{i, j}\right]=0$. Moreover,

$$
\begin{aligned}
\left|\operatorname{Var}\left[g_{\mathbf{z}}\left(\left(\mathbf{X}_{i}, \mathbf{Z}_{i}\right),\left(\mathbf{X}_{j}, \mathbf{Z}_{j}\right)\right)\right]\right| & \leq \int K_{h}^{2}\left(\mathbf{z}_{1}-\mathbf{z}\right) K_{h}^{2}\left(\mathbf{z}_{2}-\mathbf{z}\right)\left(\mathbb{1}\left\{\mathbf{x}_{1}<\mathbf{x}_{2}\right\}-\mathbb{P}\left(\mathbf{X}_{i}<\mathbf{X}_{j} \mid \mathbf{Z}_{i}=\mathbf{Z}_{j}=\mathbf{z}\right)\right)^{2} \\
\times f_{\mathbf{X}, \mathbf{Z}}\left(\mathbf{x}_{1}, \mathbf{z}_{1}\right) f_{\mathbf{X}, \mathbf{Z}}\left(\mathbf{x}_{2}, \mathbf{z}_{2}\right) d \mathbf{x}_{1} d \mathbf{x}_{2} d \mathbf{z}_{1} d \mathbf{z}_{2} & \\
& \leq \int \frac{K^{2}\left(\mathbf{t}_{1}\right) K^{2}\left(\mathbf{t}_{2}\right)}{h^{2 p}} f_{\mathbf{X}, \mathbf{Z}}\left(\mathbf{x}_{1}, \mathbf{z}-h \mathbf{t}_{1}\right) f_{\mathbf{X}, \mathbf{Z}}\left(\mathbf{x}_{2}, \mathbf{z}-h \mathbf{t}_{2}\right) d \mathbf{x}_{1} d \mathbf{x}_{2} d \mathbf{t}_{1} d \mathbf{t}_{2} \\
& \leq h^{-2 p} f_{\mathbf{Z}, \max }^{2}\left(\int K^{2}\right)^{2},
\end{aligned}
$$

where in the last line we used Assumptions 3.1(b) and 3.3. The same upper bound applies for $\tilde{g}_{i, j}$ (invoke Cauchy-Schwarz inequality). Here, we choose $b=-a=2 C_{K}^{2} h^{-2 p}$. Applying Lemma 11, for every $t>0$, we obtain

$$
\begin{aligned}
\mathbb{P}\left(\left|\sum_{1 \leq i \neq j \leq n}\left(S_{i, j}(\mathbf{z})-\mathbb{E}\left[S_{i, j}(\mathbf{z})\right]\right)\right| \geq t\right) & \leq \mathbb{P}\left(\frac{2}{n(n-1)} \sum_{1 \leq i<j \leq n} \tilde{g}_{i, j}>t\right) \\
& \leq 2 \exp \left(-\frac{[n / 2] t^{2}}{2 h^{-2 p} f_{\mathbf{Z}, \text { max }}^{2}\left(\int K^{2}\right)^{2}+(4 / 3) C_{K}^{2} h^{-2 p} t}\right) .
\end{aligned}
$$

The latter inequality, (19) and (20) yield the result. 


\section{A.5 Proof of Proposition 4}

We will prove the following lemma, that straightforwardly implies the result.

Lemma 15. Under the assumptions and conditions of Proposition 4, we have

$$
\begin{aligned}
& \mathbb{P}\left(\left|\sum_{1 \leq i, j \leq n} S_{i, j}(\mathbf{z})\right|>\frac{C_{\mathbf{X Z}, \alpha} h^{\alpha}}{\alpha !}+\frac{3 f_{\mathbf{z}}(\mathbf{z}) \int K^{2}}{2 n h^{p}}+t\right) \\
& \quad \leq \quad C_{2} \exp \left(-\frac{\alpha_{2} n h^{p} t}{8 f_{\mathbf{Z}, \max }\left(\int K^{2}\right)}\right)+2 \exp \left(-\frac{n h^{p}\left(f_{\mathbf{z}}(\mathbf{z})-C_{\tilde{K}, 2} h^{2}\right)^{2}}{8 f_{\mathbf{Z}, \max } \int \tilde{K}^{2}+4 C_{\tilde{K}}\left(f_{\mathbf{z}}(\mathbf{z})-C_{\tilde{K}, 2} h^{2}\right) / 3}\right) \\
& \quad+2 \exp \left(\frac{n h^{p} t^{2}}{32 \int K^{2}\left(\int|K|\right)^{2} f_{\mathbf{Z}, \max }^{3}+8 C_{K} \int|K| f_{\mathbf{Z}, \text { max }} t / 3}\right) .
\end{aligned}
$$

Proof : As in the proof of Lemma 14, we will use the decomposition of $\sum_{1 \leq i, j \leq n} S_{i, j}(\mathbf{z})$ given in Equation (15) and bound separately each of its three components with high probability. We keep the same bounds for the diagonal term $\Delta_{n}$ from Equation (19) and for the bias term in Equation (20). The difference from Lemma 14 will come from the treatment of the stochastic term that is detailed below.

Now, we consider the function $\ell_{\mathbf{z}}$ defined in Equation (13). Note that $\left\|\ell_{\mathbf{z}}\right\|_{\infty} \leq 1$. By usual changes of variables, we get

$$
\begin{gathered}
\int \ell_{\mathbf{z}}^{2}\left(\mathbf{x}_{1}, \mathbf{z}_{1}, \mathbf{x}_{2}, \mathbf{z}_{2}\right) f_{\mathbf{X}, \mathbf{Z}}\left(\mathbf{x}_{1}, \mathbf{z}_{1}\right) f_{\mathbf{X}, \mathbf{Z}}\left(\mathbf{x}_{2}, \mathbf{z}_{2}\right) d \mathbf{x}_{1} d \mathbf{x}_{2} d \mathbf{z}_{1} d \mathbf{z}_{2} \\
\leq 3 h^{2 p} \frac{\left(\int K^{2} f_{\mathbf{z}, \max }\right)^{2}}{\left(4 C_{K}^{2}\right)^{2}}+6 h^{3 p} \frac{\int K^{2} f_{\mathbf{z}, \max }\left(\int|K| f_{\mathbf{z}, \text { max }}\right)^{2}}{\left(4 C_{K}^{2}\right)^{2}} \leq \sigma^{2}, \text { with } \\
\sigma:=h^{p} C_{\sigma}, C_{\sigma}:=\int K^{2} f_{\mathbf{z}, \max } /\left(2 C_{K}^{2}\right),
\end{gathered}
$$

because $6 h^{p} \int K^{2} f_{\mathbf{z}, \max }\left(\int|K| f_{\mathbf{z}, \max }\right)^{2} \leq\left(\int K^{2} f_{\mathbf{z} \text {, } \max }\right)^{2}$ and because of Assumptions 3.1(b) and 3.3. With the notations of [26], this implies $D=1, m=1$ and $L$ is arbitrarily small. Therefore, Theorem 2 in [26] yields

$$
\mathbb{P}\left(\frac{1}{2 n}\left|\sum_{i \neq j} \ell_{\mathbf{z}}\left(\mathbf{X}_{i}, \mathbf{Z}_{i}, \mathbf{X}_{j}, \mathbf{Z}_{j}\right)\right|>x\right) \leq C_{2} \exp \left(-\frac{\alpha_{2} x}{\sigma}\right),
$$

for some universal constants $C_{2}$ and $\alpha_{2}$ when $x \leq n \sigma^{3}$. By setting $t / 2=4 C_{K}^{2} x /\left(n h^{2 p}\right)$ and applying Lemma 11, this provides

$$
\begin{aligned}
& \mathbb{P}\left(\left|\sum_{1 \leq i \neq j \leq n}\left(S_{i, j}(\mathbf{z})-\mathbb{E}\left[S_{i, j}(\mathbf{z})\right]\right)\right| \geq t\right) \leq \mathbb{P}\left(\frac{1}{n^{2}}\left|\sum_{1 \leq i \neq j \leq n} \xi_{i j}\right| \geq t / 2\right)+\mathbb{P}\left(\left|\frac{1}{n} \sum_{i=1}^{n} \bar{g}_{i}\right| \geq t / 4\right) \\
& \quad \leq \quad C_{2} \exp \left(-\frac{\alpha_{2} n t h^{p}}{8 f_{\mathbf{Z}, \text { max }}\left(\int K^{2}\right)}\right)+2 \exp \left(\frac{n h^{p} t^{2}}{32 \int K^{2}\left(\int|K|\right)^{2} f_{\mathbf{Z}, \text { max }}^{3}+8 / 3 C_{K} \int|K| f_{\mathbf{Z}, \text { max }} t}\right),
\end{aligned}
$$

when $t \leq 2 h^{p}\left(\int K^{2}\right)^{3} f_{\mathbf{Z} \text {, max }}^{3} / C_{K}^{4}$. Combining this inequality, (19) and (20) with the decomposition (15) conclude the proof.

\section{A.6 Proof of Proposition 6}

For $k=1$, we follow the path of the proof of Proposition 4 (of Section A.5). Since $\hat{\tau}_{1,2 \mid \mathbf{Z}=\mathbf{z}}-$ $\tau_{1,2 \mid \mathbf{Z}=\mathbf{z}}=4 \sum_{1 \leq i, j \leq n} S_{i, j}(\mathbf{z}) / \hat{f}_{\mathbf{Z}}^{2}(\mathbf{z})$ (by Equation 14), we prove the result if we bound from above $1 / \hat{f}_{\mathbf{Z}}^{2}(\mathbf{z})$ and 
$\left|\sum_{1 \leq i, j \leq n} S_{i, j}(\mathbf{z})\right|$ uniformly w.r.t. $\mathbf{z} \in \mathcal{Z}$. To be specific, for any positive constant $\mu<1$, if $\left|\hat{f}_{\mathbf{Z}}(\mathbf{z})-f_{\mathbf{Z}}(\mathbf{z})\right| \leq \mu f_{\mathbf{z}, \min }$, then $1 / \hat{f}_{\mathbf{Z}}^{2}(\mathbf{z}) \leq f_{\mathbf{z}, \min }^{-2}(1-\mu)^{-2}$. We deduce

$$
\mathbb{P}\left(\sup _{\mathbf{z} \in \mathcal{Z}}\left|\hat{\tau}_{1,2 \mid \mathbf{Z}=\mathbf{z}}-\tau_{1,2 \mid \mathbf{Z}=\mathbf{z}}\right|>x\right) \leq \mathbb{P}\left(|| \hat{f}_{\mathbf{Z}}-f_{\mathbf{Z}} \|_{\infty}>\mu f_{\mathbf{z}, \text { min }}\right)+\mathbb{P}\left(\frac{4}{f_{\mathbf{Z}, \text { min }}^{2}(1-\mu)^{2}} \sup _{\mathbf{z} \in \mathcal{Z}}\left|\sum_{1 \leq i, j \leq n} S_{i, j}(\mathbf{z})\right|>x\right) .
$$

First invoke the uniform exponential inequality, as stated in [35], Proposition 9: for every $\varepsilon<$ $b_{K} \int K^{2} f_{\mathbf{Z}, \max } / C_{K}$,

$$
\mathbb{P}\left(\left\|\hat{f}_{\mathbf{Z}}-f_{\mathbf{Z}}\right\|_{\infty}>\varepsilon+\frac{C_{\mathbf{X Z}, \alpha} h^{\alpha}}{\alpha !}\right) \leq \mathbb{P}\left(\left\|\hat{f}_{\mathbf{Z}}-\mathbb{E}\left[\hat{f}_{\mathbf{Z}}\right]\right\|_{\infty}>\varepsilon\right) \leq L_{K} \exp \left(-C_{f, K} n h^{p} \varepsilon^{2}\right),
$$

for $n$ sufficiently large. Then, apply Lemma 16 , by setting $(x, \varepsilon)$ so that

$$
x=\frac{4}{f_{\mathbf{z}, \min }^{2}(1-\mu)^{2}}\left(\frac{C_{\mathbf{X Z}, \alpha} h^{\alpha}}{\alpha !}+\frac{3 f_{\mathbf{z}, \max } \int K^{2}}{2 n h^{p}}+t\right) \text { and } \varepsilon+\frac{C_{\mathbf{X Z}, \alpha} h^{\alpha}}{\alpha !}=\mu f_{\mathbf{z}, \min } .
$$

Lemma 16. Under the assumptions and conditions of Proposition 6, we have

$$
\begin{aligned}
& \mathbb{P}\left(\sup _{\mathbf{z} \in Z}\left|\sum_{1 \leq i, j \leq n} S_{i, j}(\mathbf{z})\right|>\frac{C_{\mathbf{X Z}, \alpha} h^{\alpha}}{\alpha !}+\frac{3 f_{\mathbf{z}, \max } \int K^{2}}{2 n h^{p}}+t\right) \leq C_{2} D \exp \left(-\frac{\alpha_{2} n t h^{p}}{8 f_{\mathbf{z}, \max }\left(\int K^{2}\right)}\right) \\
& \quad+L_{\tilde{K}} \exp \left(-\frac{C_{f, \tilde{K}} n h^{p}\left(f_{\mathbf{z}, \max }-\tilde{C}_{\mathbf{X Z}, 2} h^{2}\right)^{2}}{4}\right)+2 \exp \left(-\frac{A_{2} n h^{p} t^{2} C_{K}^{-4}}{16^{2} A_{1}^{2} \int K^{2} f_{\mathbf{z}, \max }^{3}\left(\int|K|\right)^{2}}\right)+2 \exp \left(-\frac{A_{2} n h^{p} t}{16 C_{K}^{2} A_{1}}\right) .
\end{aligned}
$$

Proof: We will use the same arguments as in the proofs of Lemmas 14 and 15. We still invoke the decomposition (15). First let us find a uniform bound for the "diagonal term" $\Delta_{n}(\mathbf{z})=\sum_{i=1}^{n} S_{i, i}(\mathbf{z})=\int K^{2} \tilde{f}_{\mathbf{z}}(\mathbf{z}) /\left(n h^{p}\right)$. As in (24), for every $\varepsilon<b_{\tilde{K}} \int \tilde{K}^{2} f_{\mathbf{Z}, \max } / C_{\tilde{K}}$,

$$
\mathbb{P}\left(\left\|\tilde{f}_{\mathbf{Z}}-f_{\mathbf{Z}}\right\|_{\infty}>\varepsilon+\frac{\tilde{C}_{\mathbf{X Z}, 2} h^{2}}{2}\right) \leq L_{\tilde{K}} \exp \left(-C_{f, \tilde{K}} n h^{p} \varepsilon^{2}\right),
$$

for $n$ sufficiently large. This implies

$$
\mathbb{P}\left(\sup _{\mathbf{z} \in \mathcal{Z}}\left|\frac{\int K^{2}}{n^{2} h^{p}} \sum_{i=1}^{n} \tilde{K}_{h}\left(\mathbf{Z}_{i}-\mathbf{z}\right)-\frac{f_{\mathbf{Z}}(\mathbf{z}) \int K^{2}}{n h^{p}}\right| \geq\left(\frac{\int K^{2}}{n h^{p}}\right)\left(\varepsilon+\frac{\tilde{C}_{\mathbf{X Z}, 2} h^{2}}{2}\right)\right) \leq L_{\tilde{K}} \exp \left(-C_{f, \tilde{K}} n h^{p} \varepsilon^{2}\right) .
$$

Choose $\varepsilon$ s.t. $\tilde{C}_{\mathrm{XZ}, 2} h^{2} / 2+\varepsilon=f_{\mathrm{z}, \max } / 2$ so that we have the bound

$$
\mathbb{P}\left(\sup _{\mathbf{z} \in \mathcal{Z}}\left|\Delta_{n}(\mathbf{z})\right| \geq 3 f_{\mathbf{z}, \max } \int K^{2} /\left(2 n h^{p}\right)\right) \leq L_{\tilde{K}} \exp \left(-C_{f, \tilde{K}} n h^{p} \varepsilon^{2}\right) .
$$

Second, it is easy to see that the bias term is uniformly bounded by

$$
\sup _{\mathbf{z} \in Z}\left|\mathbb{E}\left[S_{1,2}(\mathbf{z})\right]\right| \leq C_{\mathbf{X Z}, \alpha} h^{\alpha} /\left(n^{2} \alpha !\right) .
$$

Third, we bound the two components of the stochastic term given by (17). Now apply Theorem 1 in [26], by recalling (13) and considering the family $\mathcal{F}:=\left\{\ell_{\mathbf{z}}, \mathbf{z} \in z\right\}$, for a fixed bandwidth $h$. The constant $\sigma$ has the same value as in (22). It is easy to check that the latter class of functions is $L^{2}$ dense (see [26]). Set $\varepsilon \in(0,1)$. Since $K$ is $\lambda_{K}$-Lipschitz by Assumption 3.5, every function $\ell_{\mathbf{z}} \in \mathcal{F}$ can be approximated in $L^{2}$ by a function $\ell_{\mathbf{z}_{j}} \in \mathcal{F}$, for some $j \in\{1, \ldots, m\}$ s.t. $\int\left|\ell_{\mathbf{z}}-\ell_{\mathbf{z}_{i}}\right|^{2} d v \leq \varepsilon^{2}$, for any probability measure $v$. Indeed, $\int\left|\ell_{\mathbf{z}}-\ell_{\mathbf{z}_{i}}\right|^{2} d v \leq 64 \lambda_{K}^{2}\left\|\mathbf{z}-\mathbf{z}_{j}\right\|_{\infty}^{2} C_{K}^{2} h^{-2}$ that is less than $\varepsilon^{2}$, if we cover $z$ by a grid of $m$ points $\left(\mathbf{z}_{j}\right)$ in $z$ s.t. $\left\|\mathbf{z}-\mathbf{z}_{j}\right\|_{\infty} \leq \varepsilon h /\left(8 C_{K} \lambda_{K}\right):=\varepsilon \delta$. This can be done with $m \leq \varepsilon^{-p}\left\lceil\prod_{k=1}^{p}\left(\left(b_{k}-a_{k}\right) / \delta\right)\right\rceil=\varepsilon^{-p}\left\lceil\mathcal{V} \delta^{-p}\right\rceil$ points. Then, with the notations of [26], $L=p$ and $D=\mathcal{V}\left(8 C_{K} \lambda_{K} / h\right)^{p}$. As above, this yields

$$
\left.\mathbb{P}\left(\sup _{\mathbf{z} \in \mathcal{Z}} \frac{1}{n^{2}} \mid \sum_{1 \leq i \neq j \leq n} \xi_{\mathbf{Z}}\left(\mathbf{X}_{i}, \mathbf{Z}_{i}, \mathbf{X}_{j}, \mathbf{Z}_{j}\right)\right) \mid>t\right) \leq C_{2} D \exp \left(-\frac{\alpha_{2} n h^{p} t}{8 f_{\mathbf{Z}, \max }} \int K^{2}\right),
$$


when $t \leq 2 h^{p}\left(\int K^{2}\right)^{3} f_{\mathbf{Z}, \max }^{3} / C_{K}^{4}$.

It remains to bound $\mathbb{P}\left(\sup _{\mathbf{z} \in \mathcal{Z}}\left|n^{-1} \sum_{i=1}^{n} \bar{g}_{i}\right|>t / 4\right)$. Consider the family of functions

$$
\mathcal{F}:=\left\{\left(\mathbf{x}_{1}, \mathbf{z}_{1}\right) \in \mathbb{R} \times Z \mapsto \frac{h^{p}}{4 C_{K}^{2}} \mathbb{E}\left[g_{\mathbf{z}}\left(\mathbf{x}_{1}, \mathbf{z}_{1}, \mathbf{X}, \mathbf{Z}\right)\right], \mathbf{z} \in Z\right\} .
$$

This family of functions is bounded by one and its variance is less than $\bar{\sigma}^{2}:=h^{p} \int K^{2} f_{\mathbf{z}, \max }^{3}\left(\int|K|\right)^{2}$. Therefore, using Assumption 3.5, we can apply Propositions 9 and 10 in [12] that are coming from [11]: for some universal constants $A_{1}$ and $A_{2}$, some constant $A_{\bar{g}}$ that depends on $K$ and $f_{\mathbf{z}, \max }$ (see Proposition 1 in [11]) and for every $x>0$,

$$
\mathbb{P}\left(\sup _{\mathbf{z} \in \mathcal{Z}} \frac{h^{p}}{4 C_{K}^{2}}\left|\sum_{i=1}^{n} \mathbb{E}\left[g_{\mathbf{z}}\left(\mathbf{X}_{i}, \mathbf{Z}_{i}, \mathbf{X}, \mathbf{Z}\right) \mid \mathbf{X}_{i}, \mathbf{Z}_{i}\right]\right|>A_{1}\left(x+A_{\bar{g}} n^{1 / 2} \bar{\sigma} \ln (1 / \bar{\sigma})\right)\right) \leq 2 \exp \left(-\frac{A_{2} x^{2}}{n \bar{\sigma}^{2}}\right)+2 e^{-A_{2} x},
$$

that can be rewritten as

$$
\mathbb{P}\left(\sup _{\mathbf{z} \in \mathcal{Z}} \frac{1}{n}\left|\sum_{i=1}^{n} \bar{g}_{i}\right|>4 A_{1} C_{K}^{2}\left(x-\frac{A_{\bar{g}} \bar{\sigma}}{n^{1 / 2} h^{p}} \ln (\bar{\sigma})\right)\right) \leq 2 \exp \left(-\frac{A_{2} n h^{2 p} \chi^{2}}{\bar{\sigma}^{2}}\right)+2 \exp \left(-A_{2} n h^{p} x\right) .
$$

For any positive $t$ s.t. $4 A_{1} C_{K}^{2}(n-1) A_{\bar{g}} \bar{\sigma} \ln (1 / \bar{\sigma})<n^{3 / 2} h^{p} t / 8$, note that we can find a real $x>t h^{p} /\left(16 C_{K}^{2} A_{1}\right)$. Then, we have

$$
\mathbb{P}\left(\sup _{\mathbf{z} \in \mathcal{Z}} \frac{(n-1)}{n^{2}}\left|\sum_{i=1}^{n} \bar{g}_{i}\right|>\frac{t}{4}\right) \leq 2 \exp \left(-\frac{A_{2} n h^{p} t^{2} C_{K}^{-4}}{16^{2} A_{1}^{2} \int K^{2} f_{\mathbf{Z}, \max }^{3}\left(\int|K|\right)^{2}}\right)+2 \exp \left(-\frac{A_{2} n h^{p} t}{16 C_{K}^{2} A_{1}}\right) .
$$

The proof is completed by combining all the inequalities (25), (26), (27) and (28) with the decompositions (15) and (17).

\section{A.7 Proof of Proposition 7}

Note that $\tau_{1,2 \mid \mathbf{Z}=\mathbf{z}}=\mathbb{E}\left[g_{k}\left(\mathbf{X}_{1}, \mathbf{X}_{2}\right) \mid \mathbf{Z}_{1}=\mathbf{z}, \mathbf{Z}_{2}=\mathbf{z}\right]$ for every $k=1$, 2, 3, and that our estimators with the weights (2) can be written as $\hat{\tau}_{1,2 \mid \mathbf{Z}=\mathbf{z}}^{(k)}:=U_{n}\left(g_{k}\right) /\left\{U_{n}(1)+\epsilon_{n}\right\}$, where

$$
U_{n}(g):=\frac{1}{n(n-1)} \sum_{1 \leq i \neq j \leq n} g\left(\mathbf{X}_{i}, \mathbf{X}_{j}\right) \frac{K_{h}\left(\mathbf{z}-\mathbf{Z}_{i}\right) K_{h}\left(\mathbf{z}-\mathbf{Z}_{j}\right)}{\mathbb{E}\left[K_{h}(\mathbf{z}-\mathbf{Z})\right]^{2}}=: \frac{1}{n(n-1)} \sum_{1 \leq i \neq j \leq n} g_{i, j},
$$

for any measurable bounded function $g$, with the residual diagonal term $\epsilon_{n}:=\sum_{i=1}^{n} K_{h}^{2}\left(\mathbf{z}-\mathbf{Z}_{i}\right) /\{n(n-$ 1) $\left.\mathbb{E}\left[K_{h}(\mathbf{z}-\mathbf{Z})\right]^{2}\right\}$. By Bochner's lemma (see Bosq and Lecoutre [5]), $\epsilon_{n}$ is $O_{P}\left(\left(n h^{p}\right)^{-1}\right)$, and it will be negligible compared to $U_{n}(1)$. Since the reasoning will be exactly the same for every estimator $\tau_{1,2 \mid \mathbf{z}}^{(k)}$, i.e. for every function $g_{k}, k=1,2,3$, we omit the sub-index $k$. Then, the functions $g_{k}$ will be simply denoted by $g$.

The expectation of our U-statistics is

$$
\begin{aligned}
\mathbb{E} & {\left[U_{n}(g)\right]:=\mathbb{E}\left[g\left(\mathbf{X}_{1}, \mathbf{X}_{2}\right) K_{h}\left(\mathbf{z}-\mathbf{Z}_{1}\right) K_{h}\left(\mathbf{z}-\mathbf{Z}_{2}\right)\right] / \mathbb{E}\left[K_{h}(\mathbf{z}-\mathbf{Z})\right]^{2} } \\
& =\int g\left(\mathbf{x}_{1}, \mathbf{x}_{2}\right) K\left(\mathbf{t}_{1}\right) K\left(\mathbf{t}_{2}\right) f_{\mathbf{X}, \mathbf{Z}}\left(\mathbf{x}_{1}, \mathbf{z}+h \mathbf{t}_{1}\right) f_{\mathbf{X}, \mathbf{Z}}\left(\mathbf{x}_{2}, \mathbf{z}+h \mathbf{t}_{2}\right) d \mathbf{x}_{1} d \mathbf{x}_{2} d \mathbf{t}_{1} d \mathbf{t}_{2} / \mathbb{E}\left[K_{h}(\mathbf{z}-\mathbf{Z})\right]^{2} \\
& \rightarrow \frac{1}{f_{\mathbf{Z}}^{2}(\mathbf{z})} \int g\left(\mathbf{x}_{1}, \mathbf{x}_{2}\right) f_{\mathbf{X}, \mathbf{Z}}\left(\mathbf{x}_{1}, \mathbf{z}\right) f_{\mathbf{X}, \mathbf{Z}}\left(\mathbf{x}_{2}, \mathbf{z}\right) d \mathbf{x}_{1} d \mathbf{x}_{2}=\mathbb{E}\left[g\left(\mathbf{X}_{1}, \mathbf{X}_{2}\right) \mid \mathbf{Z}_{1}=\mathbf{z}, \mathbf{Z}_{2}=\mathbf{z}\right],
\end{aligned}
$$

applying Bochner's lemma to $\mathbf{z} \mapsto \int g\left(\mathbf{x}_{1}, \mathbf{x}_{2}\right) f_{\mathbf{X} \mid \mathbf{Z}=\mathbf{z}}\left(\mathbf{x}_{1}\right) f_{\mathbf{X} \mid \mathbf{Z}=\mathbf{z}}\left(\mathbf{x}_{2}\right) d \mathbf{x}_{1} d \mathbf{x}_{2}=\tau_{1,2 \mid \mathbf{Z}=\mathbf{z}}$, that is a continuous function by assumption. 
Set $\theta_{n}:=\mathbb{E}\left[U_{n}(g)\right], g^{\star}\left(\mathbf{x}_{1}, \mathbf{x}_{2}\right):=\left(g\left(\mathbf{x}_{1}, \mathbf{x}_{2}\right)+g\left(\mathbf{x}_{2}, \mathbf{x}_{1}\right)\right) / 2$ and $g_{i, j}^{\star}=\left(g_{i, j}+g_{j, i}\right) / 2$ for every $(i, j)$, $i \neq j$. Note that $U_{n}(g)=U_{n}\left(g^{\star}\right)$. Since $g^{\star}$ is symmetrical, the HÃąjek projection $\hat{U}_{n}\left(g^{\star}\right)$ of $U_{n}\left(g^{\star}\right)$ satisfies $\hat{U}_{n}\left(g^{\star}\right):=2 \sum_{j=1}^{n} \mathbb{E}\left[g_{0, j}^{\star} \mid \mathbf{X}_{j}, \mathbf{Z}_{j}\right] / n-\theta_{n}$. Note that $\mathbb{E}\left[\hat{U}_{n}\left(g^{*}\right)\right]=\theta_{n}=\tau_{1,2 \mid \mathbf{Z}=\mathbf{z}}+o_{P}(1)$. Since $\operatorname{Var}\left(\hat{U}_{n}\left(g^{\star}\right)=\right.$ $4 \operatorname{Var}\left(\mathbb{E}\left[g_{0, j}^{\star} \mid \mathbf{X}_{j}, \mathbf{Z}_{j}\right]\right) / n=O\left(\left(n h^{p}\right)^{-1}\right)$, then $\hat{U}_{n}\left(g^{\star}\right)=\theta_{n}+o_{P}(1)=\tau_{1,2 \mid \mathbf{Z}=\mathbf{z}}+o_{P}(1)$.

Moreover, using the notation $\bar{g}_{i, j}:=g_{i, j}^{\star}-\mathbb{E}\left[g_{i, j}^{\star} \mid \mathbf{X}_{j}, \mathbf{Z}_{j}\right]-\mathbb{E}\left[g_{i, j}^{\star} \mid \mathbf{X}_{i}, \mathbf{Z}_{i}\right]+\theta_{n}$ for $1 \leq i \neq j \leq n$, we have $U_{n}\left(g^{\star}\right)-\hat{U}_{n}\left(g^{\star}\right)=\sum_{1 \leq i \neq j \leq n} \bar{g}_{i, j} / n(n-1)$. By usual U-statistics calculations, it can be easily checked that

$$
\operatorname{Var}\left(U_{n}\left(g^{\star}\right)-\hat{U}_{n}\left(g^{\star}\right)\right)=\frac{1}{n^{2}(n-1)^{2}} \sum_{1 \leq i_{1} \neq j_{1} \leq n} \sum_{1 \leq i_{2} \neq j_{2} \leq n} \mathbb{E}\left[\bar{g}_{i_{1}, j_{1}} \bar{g}_{i_{2}, j_{2}}\right]=O\left(\frac{1}{n^{2} h^{2 p}}\right) .
$$

Indeed, when all indices $\left(i_{1}, i_{2}, j_{1}, j_{2}\right)$ are different, or when there is a single identity among them, $\mathbb{E}\left[\bar{g}_{i_{1}, j_{1}} \bar{g}_{i_{2}, j_{2}}\right]$ is zero. The first nonzero terms arise when there are two identities among the indices, i.e. $i_{1}=i_{2}$ and $j_{1}=j_{2}$ (or $i_{1}=j_{2}$ and $j_{1}=i_{2}$ ). In the latter case, we get an upper bound as $O\left(\left(n h^{p}\right)^{-2}\right)$ when $f_{\mathrm{Z}}$ is continuous at $\mathbf{z}$, by usual changes of variable techniques and Bochner's Lemma. Then, $U_{n}\left(g^{\star}\right)=\hat{U}_{n}\left(g^{\star}\right)+o_{P}(1)=$ $\tau_{1,2 \mid \mathbf{Z}=\mathbf{z}}+o_{P}(1)$. Note that $U_{n}(1)+\epsilon_{n}$ tends to one in probability (Bochner's lemma). As a consequence, $\hat{\tau}_{1,2 \mid \mathbf{Z}=\mathbf{z}}=U_{n}\left(g^{\star}\right) /\left(U_{n}(1)+\epsilon_{n}\right)$ tends to $\tau_{1,2 \mid \mathbf{Z}=\mathbf{z}} / 1$ by the continuous mapping theorem.

\section{A.8 Proof of Proposition 8}

Let us note that

$$
\tau_{1,2 \mid \mathbf{Z}=\mathbf{z}}=\mathbb{E}\left[g_{k}\left(\mathbf{X}_{1}, \mathbf{X}_{2}\right) \mid \mathbf{Z}_{1}=\mathbf{z}, \mathbf{Z}_{2}=\mathbf{z}\right]=\int g_{k}\left(\mathbf{x}_{1}, \mathbf{x}_{2}\right) f_{\mathbf{X} \mid \mathbf{Z}=\mathbf{z}}\left(\mathbf{x}_{1}\right) f_{\mathbf{X} \mid \mathbf{Z}=\mathbf{z}}\left(\mathbf{x}_{2}\right) d \mathbf{x}_{1} d \mathbf{x}_{2}=\frac{\phi_{k}(\mathbf{z})}{f_{\mathbf{Z}}^{2}(\mathbf{z})},
$$

where $\phi_{k}(\mathbf{z}):=\int g_{k}\left(\mathbf{x}_{1}, \mathbf{x}_{2}\right) f_{\mathbf{X}, \mathbf{Z}}\left(\mathbf{x}_{1}, \mathbf{z}\right) f_{\mathbf{X}, \mathbf{Z}}\left(\mathbf{x}_{2}, \mathbf{z}\right) d \mathbf{x}_{1} d \mathbf{x}_{2}$. Also write $\hat{\tau}_{1,2 \mid \mathbf{Z}=\mathbf{z}}^{(k)}=\hat{\phi}_{k}(\mathbf{z}) / \hat{f}_{\mathbf{Z}}^{2}(\mathbf{z})$, where $\hat{\phi}_{k}(\mathbf{z}):=$ $n^{-2} \sum_{i, j=1}^{n} K_{h}\left(\mathbf{Z}_{i}-\mathbf{z}\right) K_{h}\left(\mathbf{Z}_{j}-\mathbf{z}\right) g_{k}\left(\mathbf{X}_{i}, \mathbf{X}_{j}\right)$ and $\hat{f}_{\mathbf{Z}}(\mathbf{z}):=n^{-1} \sum_{i=1}^{n} K_{h}\left(\mathbf{Z}_{i}-\mathbf{z}\right)$. Therefore, we have

$$
\hat{\tau}_{1,2 \mid \mathbf{Z}=\mathbf{z}}^{(k)}-\tau_{1,2 \mid \mathbf{Z}=\mathbf{z}}=\frac{\hat{\phi}_{k}(\mathbf{z})-\phi_{k}(\mathbf{z})}{\hat{f}_{\mathbf{Z}}^{2}(\mathbf{z})}-\tau_{1,2 \mid \mathbf{Z}=\mathbf{z}} \frac{\hat{f}_{\mathbf{Z}}(\mathbf{z})-f_{\mathbf{Z}}(\mathbf{z})}{\hat{f}_{\mathbf{Z}}^{2}(\mathbf{z})} \times\left(\hat{f}_{\mathbf{Z}}(\mathbf{z})+f_{\mathbf{Z}}(\mathbf{z})\right) .
$$

By usual uniform consistency results (see for example Bosq and Lecoutre [5]), $\sup _{\mathbf{z} \in Z}\left|\hat{f}_{\mathbf{Z}}(\mathbf{z})-f_{\mathbf{Z}}(\mathbf{z})\right| \rightarrow 0$ almost surely, as $n \rightarrow \infty$. We deduce that

$$
\min _{\mathbf{z} \in \mathcal{Z}} \hat{f}_{\mathbf{Z}}^{2}(\mathbf{z}) \geq f_{\mathbf{Z}, \min }^{2} / 2 \text {, and } \max _{\mathbf{z} \in \mathcal{Z}}\left|\hat{f}_{\mathbf{Z}}(\mathbf{z})+f_{\mathbf{Z}}(\mathbf{z})\right| \leq 2 \max _{\mathbf{z} \in \mathcal{Z}} f_{\mathbf{Z}}(\mathbf{z}) \text { a.s. }
$$

This means it is sufficient to prove the uniform strong consistency of $\hat{\phi}_{k}$ on $\mathcal{Z}$, to obtain that $\sup _{\mathbf{z} \in \mathcal{Z}} \mid \hat{\tau}_{1,2 \mid \mathbf{Z}=\mathbf{z}}^{(k)}{ }^{-}$ $\tau_{1,2 \mid \mathbf{Z}=\mathbf{z}}^{(k)} \mid$ tends to zero a.s.

Note that, by Bochner's Lemma, $\sup _{\mathbf{z} \in \mathcal{Z}}\left|\mathbb{E}\left[\hat{\phi}_{k}(\mathbf{z})\right]-\phi_{k}(\mathbf{z})\right| \rightarrow 0$. Then, it remains to show that $\sup _{\mathbf{z} \in Z}\left|\hat{\phi}_{k}(\mathbf{z})-\mathbb{E}\left[\hat{\phi}_{k}(\mathbf{z})\right]\right| \rightarrow 0$ almost surely. Let $\rho_{n}>0$ be such that we cover $z$ by the union of $l_{n}$ open balls $B\left(\mathbf{t}_{l}, \rho_{n}\right)$, where $\mathbf{t}_{1}, \ldots, \mathbf{t}_{l_{n}} \in \mathbb{R}^{p}$ and $l_{n} \in \mathbb{N}^{\star}$. Then

$$
\sup _{\mathbf{z} \in \mathcal{Z}}\left|\hat{\phi}_{k}(\mathbf{z})-\mathbb{E}\left[\hat{\phi}_{k}(\mathbf{z})\right]\right| \leq \sup _{l=1, \ldots l_{n}}\left|\hat{\phi}_{k}\left(\mathbf{t}_{l}\right)-\mathbb{E}\left[\hat{\phi}_{k}\left(\mathbf{t}_{l}\right)\right]\right|+A_{n},
$$

where $A_{n}:=\sup _{l=1, \ldots l_{n}} \sup _{\mathbf{z} \in B\left(\mathbf{t}_{l}, \rho_{n}\right)}\left|\hat{\phi}_{k}(\mathbf{z})-\hat{\phi}_{k}\left(\mathbf{t}_{l}\right)-\left(\mathbb{E}\left[\hat{\phi}_{k}(\mathbf{z})\right]-\mathbb{E}\left[\hat{\phi}_{k}\left(\mathbf{t}_{l}\right)\right]\right)\right|$. For any index $l \in\left\{1, \ldots, l_{n}\right\}$ and any $\mathbf{z} \in B\left(\mathbf{t}_{l}, \rho_{n}\right)$, a first-order expansion yields

$$
\begin{aligned}
& \left|\hat{\phi}_{k}(\mathbf{z})-\hat{\phi}_{k}\left(\mathbf{t}_{l}\right)-\left(\mathbb{E}\left[\hat{\phi}_{k}(\mathbf{z})\right]-\mathbb{E}\left[\hat{\phi}_{k}\left(\mathbf{t}_{l}\right)\right]\right)\right| \\
& =\mid \frac{1}{n(n-1)} \sum_{1 \leq i \neq j \leq n} g_{k}\left(\mathbf{X}_{i}, \mathbf{X}_{j}\right) K_{h}\left(\mathbf{z}-\mathbf{Z}_{i}\right) K_{h}\left(\mathbf{z}-\mathbf{Z}_{j}\right)
\end{aligned}
$$




$$
\begin{aligned}
& -\frac{1}{n(n-1)} \sum_{1 \leq i \neq j \leq n} g_{k}\left(\mathbf{X}_{i}, \mathbf{X}_{j}\right) K_{h}\left(\mathbf{t}_{l}-\mathbf{Z}_{i}\right) K_{h}\left(\mathbf{t}_{l}-\mathbf{Z}_{j}\right) \\
& -\left(\mathbb{E}\left[g_{k}\left(\mathbf{X}_{1}, \mathbf{X}_{2}\right) K_{h}\left(\mathbf{z}-\mathbf{Z}_{1}\right) K_{h}\left(\mathbf{z}-\mathbf{Z}_{2}\right)\right]-\mathbb{E}\left[g_{k}\left(\mathbf{X}_{i}, \mathbf{X}_{j}\right) K_{h}\left(\mathbf{t}_{l}-\mathbf{Z}_{i}\right) K_{h}\left(\mathbf{t}_{l}-\mathbf{Z}_{j}\right)\right]\right) \mid \\
\leq & \frac{C_{L i p, K}}{h^{2 p+1}}\left|\mathbf{z}-\mathbf{t}_{l}\right|\left(\mathbb{E}\left[\left|g_{k}\left(\mathbf{X}_{1}, \mathbf{X}_{2}\right)\right|\right]+\frac{1}{n(n-1)} \sum_{1 \leq i \neq j \leq n}\left|g_{k}\left(\mathbf{X}_{i}, \mathbf{X}_{j}\right)\right|\right) \\
= & O\left(\frac{\rho_{n}}{h^{2 p+1}}\right)=O(1),
\end{aligned}
$$

for some constant $C_{L i p, K}$ and by choosing $\rho_{n}=o\left(h_{n}^{2 p+1}\right)$. Actually, we can cover $z$ in such a way that $l_{n}=$ $O\left(h_{n}^{-p(2 p+1)}\right)$. This is always possible because $z$ is a bounded set in $\mathbb{R}^{p}$. The previous upper bound is uniform w.r.t. $l$ and $\mathbf{z} \in B\left(\mathbf{t}_{l}, \rho_{n}\right)$, proving $A_{n}=o(1)$ everywhere.

Now, for every $l \leq l_{n}$, apply Equation (21) for every $\mathbf{z}=\mathbf{t}_{l}$. For any $t>0$, this yields

$$
\mathbb{P}\left(\frac{1}{n(n-1)}\left|\sum_{i \neq j} g^{(l)}\left(\left(\mathbf{X}_{i}, \mathbf{Z}_{i}\right),\left(\mathbf{X}_{j}, \mathbf{Z}_{j}\right)\right)-\mathbb{E}\left[g^{(l)}\left(\left(\mathbf{X}_{1}, \mathbf{Z}_{1}\right),\left(\mathbf{X}_{2}, \mathbf{Z}_{2}\right)\right)\right]\right|>t\right) \leq \exp \left(-\frac{C_{0} n h_{n}^{2 p} t^{2}}{C_{1}+C_{2} t}\right),
$$

for some positive constants $C_{0}, C_{1}, C_{2}$, by setting

$$
g^{(l)}\left(\left(\mathbf{X}_{i}, \mathbf{Z}_{i}\right),\left(\mathbf{X}_{j}, \mathbf{Z}_{j}\right)\right):=g_{k}\left(\mathbf{X}_{i}, \mathbf{X}_{j}\right) K_{h}\left(\mathbf{t}_{l}-\mathbf{Z}_{i}\right) K_{h}\left(\mathbf{t}_{l}-\mathbf{Z}_{j}\right)
$$

Therefore, we deduce

$$
\mathbb{P}\left(\sup _{l=1, \ldots l_{n}}\left|\hat{\phi}_{k}\left(\mathbf{t}_{l}\right)-\mathbb{E}\left[\hat{\phi}_{k}\left(\mathbf{t}_{l}\right)\right]\right| \geq t\right) \leq C_{4} h_{n}^{-p(2 p+1)} \exp \left(-\frac{C_{0} n h_{n}^{2 p} t^{2}}{C_{1}+C_{2} t}\right),
$$

for some constant $C_{4}$. Finally, applying Borel-Cantelli lemma, $\sup _{\mathbf{z} \in Z}\left|\hat{\phi}_{k}(\mathbf{z})-\mathbb{E}\left[\hat{\phi}_{k}(\mathbf{z})\right]\right|$ tends to zero a.s., proving the result.

\section{A.9 Proof of Proposition 9}

By Markov's inequality, $\sum_{i=1}^{n} w_{i, n}^{2}(\mathbf{z})=O_{P}\left(\left(n h^{p}\right)^{-1}\right)$ for any $\mathbf{z}$, that tends to zero. Then, by Slutsky's theorem, we get an asymptotic equivalence between the limiting laws of any $\hat{\tau}_{1,2 \mid \mathbf{z}}^{(k)}, k=1,2,3$, and of their linearly transformed versions $\tilde{\tau}_{1,2 \mid \mathbf{z}}$. Thus, we will prove the asymptotic normality of $\hat{\tau}_{1,2 \mid \mathbf{z}}^{(k)}$ for some index $k=1,2,3$, simply denoted by $\hat{\tau}_{1,2 \mid \mathbf{z}}$.

Let $g^{\star}\left(\mathbf{x}_{1}, \mathbf{x}_{2}\right):=\left(g_{k}\left(\mathbf{x}_{1}, \mathbf{x}_{2}\right)+g_{k}\left(\mathbf{x}_{2}, \mathbf{x}_{1}\right)\right) / 2$ for some index $k=1,2,3$ (that will be implicit in the proof). We now study the joint behavior of $\left(\hat{\tau}_{1,2 \mid \mathbf{Z}=\mathbf{z}_{i}^{\prime}}-\tau_{1,2 \mid \mathbf{Z}=\mathbf{z}_{i}^{\prime}}\right)_{i=1, \ldots, n^{\prime}}$. We will extend Stute [38]'s approach, in the case of multivariate conditioning variable $\mathbf{z}$ and studying the joint distribution of $U$-statistics at several conditioning points. As in the proof of Proposition 7, the estimator with the weights given by (2) can be rewritten as $\hat{\tau}_{1,2 \mid \mathbf{Z}=\mathbf{z}_{i}^{\prime}}:=U_{n, i}\left(g^{\star}\right) /\left(U_{n, i}(1)+\epsilon_{n, i}\right)$, where

$$
U_{n, i}(g):=\frac{1}{n(n-1) \mathbb{E}\left[K_{h}\left(\mathbf{z}_{i}^{\prime}-\mathbf{Z}\right)\right]^{2}} \sum_{j_{1}, j_{2}=1, j_{1} \neq j_{2}}^{n} g\left(\mathbf{X}_{j_{1}}, \mathbf{X}_{j_{2}}\right) K_{h}\left(\mathbf{z}_{i}^{\prime}-\mathbf{Z}_{j_{1}}\right) K_{h}\left(\mathbf{z}_{i}^{\prime}-\mathbf{Z}_{j_{2}}\right),
$$

for any bounded measurable function $g: \mathbb{R}^{4} \rightarrow \mathbb{R}$.

Now, we prove the joint asymptotic normality of $\left(U_{n, i}(g)\right)_{i=1, \ldots, n^{\prime}}$. The HÃajek projection $\hat{U}_{n, i}(g)$ of $U_{n, i}(g)$ satisfies $\hat{U}_{n, i}(g):=2 \sum_{j=1}^{n} g_{n, i}\left(\mathbf{X}_{j}, \mathbf{Z}_{j}\right) / n-\theta_{n}$, where $\theta_{n}:=\mathbb{E}\left[U_{n, i}(g)\right]$ (see [37, Section 5.3.1]) and

$$
g_{n, i}(\mathbf{x}, \mathbf{z}):=K_{h}\left(\mathbf{z}_{i}^{\prime}-\mathbf{z}\right) \mathbb{E}\left[g(\mathbf{X}, \mathbf{x}) K_{h}\left(\mathbf{z}_{i}^{\prime}-\mathbf{Z}\right)\right] / \mathbb{E}\left[K_{h}\left(\mathbf{z}_{i}^{\prime}-\mathbf{Z}\right)\right]^{2} .
$$


Lemma 17. Under the assumptions of Proposition 9, for any measurable bounded function $g$,

$$
\left(n h^{p}\right)^{1 / 2}\left(\hat{U}_{n, i}(g)-\mathbb{E}\left[U_{n, i}(g)\right]\right)_{i=1, \ldots, n^{\prime}} \stackrel{D}{\longrightarrow} \mathcal{N}\left(0, M_{\infty}(g)\right) \text {, as } n \rightarrow \infty
$$

where, for $1 \leq i, j \leq n^{\prime}$,

$$
\left[M_{\infty}(g)\right]_{i, j}:=\frac{4 \int K^{2} \mathbb{1}_{\left\{\mathbf{z}_{i}^{\prime}=\mathbf{z}_{j}^{\prime}\right\}}}{f_{\mathbf{Z}}\left(\mathbf{z}_{i}^{\prime}\right)} \int g\left(\mathbf{x}_{1}, \mathbf{x}\right) g\left(\mathbf{x}_{2}, \mathbf{X}\right) f_{\mathbf{X} \mid \mathbf{Z}=\mathbf{z}_{i}^{\prime}}(\mathbf{x}) f_{\mathbf{X} \mid \mathbf{Z}=\mathbf{z}_{i}^{\prime}}\left(\mathbf{x}_{1}\right) f_{\mathbf{X} \mid \mathbf{Z}=\mathbf{z}_{i}^{\prime}}\left(\mathbf{x}_{2}\right) d \mathbf{x} d \mathbf{x}_{1} d \mathbf{x}_{2} .
$$

This lemma is proved in A.10. Similarly as in the proof of Lemma 2.2 in Stute [38], for every $i=1, \ldots, n^{\prime}$ and every bounded symmetrical measurable function $g$, we have $\left(n h^{p}\right)^{1 / 2} \operatorname{Var}\left[\hat{U}_{n, i}(g)-U_{n, i}(g)\right]=o(1)$, which implies

$$
\left(n h^{p}\right)^{1 / 2}\left(U_{n, i}(g)-\mathbb{E}\left[U_{n, i}(g)\right]\right)_{i=1, \ldots, n^{\prime}} \stackrel{D}{\longrightarrow} \mathcal{N}\left(0, M_{\infty}(g)\right), \text { as } n \rightarrow \infty
$$

Considering two measurable bounded functions $g_{1}$ and $g_{2}$, we have $U_{n, i}\left(c_{1} g_{1}+c_{2} g_{2}\right)=c_{1} U_{n, i}\left(g_{1}\right)+$ $c_{2} U_{n, i}\left(g_{2}\right)$ for every numbers $c_{1}, c_{2}$. By the CramÃlr-Wold device, we check that

$$
\begin{array}{r}
\left(n h^{p}\right)^{1 / 2}\left(\left(U_{n, i}\left(g_{1}\right)-\mathbb{E}\left[U_{n, i}\left(g_{1}\right)\right]\right)_{i=1, \ldots, n^{\prime}},\left(U_{n, i}\left(g_{2}\right)-\mathbb{E}\left[U_{n, i}\left(g_{2}\right)\right]\right)_{i=1, \ldots, n^{\prime}}\right) \\
\stackrel{D}{\longrightarrow} \mathcal{N}\left(0,\left[\begin{array}{cc}
M_{\infty}\left(g_{1}\right) & M_{\infty}\left(g_{1}, g_{2}\right) \\
M_{\infty}\left(g_{1}, g_{2}\right) & M_{\infty}\left(g_{2}\right)
\end{array}\right]\right),
\end{array}
$$

as $n \rightarrow \infty$, where

$$
\left[M_{\infty}\left(g_{1}, g_{2}\right)\right]_{i, j}:=\frac{4 \int K^{2} \mathbb{1}_{\left\{\mathbf{z}_{i}^{\prime}=\mathbf{z}_{j}^{\prime}\right\}}}{f_{\mathbf{Z}}\left(\mathbf{z}_{i}^{\prime}\right)} \int g_{1}\left(\mathbf{x}_{1}, \mathbf{x}\right) g_{2}\left(\mathbf{x}_{2}, \mathbf{x}\right) f_{\mathbf{X} \mid \mathbf{Z}=\mathbf{z}_{i}^{\prime}}(\mathbf{x}) f_{\mathbf{X} \mid \mathbf{Z}=\mathbf{z}_{i}^{\prime}}\left(\mathbf{x}_{1}\right) f_{\mathbf{X} \mid \mathbf{Z}=\mathbf{z}_{i}^{\prime}}\left(\mathbf{x}_{2}\right) d \mathbf{x} d \mathbf{x}_{1} d \mathbf{x}_{2}
$$

Set $\tilde{\tau}_{1,2 \mid \mathbf{Z}=\mathbf{z}_{i}^{\prime}}:=U_{n, i}\left(g^{\star}\right) / U_{n, i}(1)$. Note that $\sup _{i=1, \ldots, n^{\prime}}\left|\epsilon_{n, i}\right|=O_{P}\left(n^{-1} h^{-p}\right)$. Since $\left(n h_{n}^{p}\right)^{1 / 2}\left(\hat{\boldsymbol{\tau}}_{1,2 \mid \mathbf{Z}=\mathbf{z}_{i}^{\prime}-}\right.$ $\left.\tilde{\tau}_{1,2 \mid \mathbf{Z}=\mathbf{z}_{i}^{\prime}}\right)=O_{P}\left(\left(n h_{n}^{p}\right)^{1 / 2} \epsilon_{n, i}\right)$ is $o_{P}(1)$ by Assumption 3.6(i), it is sufficient to establish the asymptotic law of $\left(n h_{n}^{p}\right)^{1 / 2}\left(\tilde{\tau}_{1,2 \mid \mathbf{Z}=\mathbf{z}_{i}^{\prime}}-\tau_{1,2 \mid \mathbf{Z}=\mathbf{z}_{i}^{\prime}}\right)$. By a limited expansion of $f_{\mathbf{X}, \mathbf{Z}}$ w.r.t. its second argument, and under Assumption 3.4, we easily check that $\mathbb{E}\left[U_{n, i}\left(g^{\star}\right)\right]=\tau_{1,2 \mid \mathbf{Z}=\mathbf{z}_{i}^{\prime}}+r_{n, i}$, where $\left|r_{n, i}\right| \leq C_{0} h_{n}^{\alpha} / f_{\mathbf{Z}}^{2}\left(\mathbf{z}_{i}^{\prime}\right)$, for some constant $C_{0}$ that is independent of $i$. Since $\mathbb{E}\left[U_{n, i}(1)\right]=1+o\left(\left(n h^{p}\right)^{-1 / 2}\right)$ and $\mathbb{E}\left[U_{n, i}\left(g^{\star}\right)\right]=\tau_{1,2 \mid \mathbf{Z}=\mathbf{z}_{i}^{\prime}}+o\left(\left(n h_{n}^{p}\right)^{-1 / 2}\right)$ by Assumption 3.6(i), we get

$$
\begin{aligned}
&\left(n h^{p}\right)^{1 / 2}\left(\left(U_{n, i}\left(g^{\star}\right)-\tau_{1,2 \mid \mathbf{Z}=\mathbf{z}_{i}^{\prime}}\right)_{i=1, \ldots, n^{\prime}},\left(U_{n, i}(1)-1\right)_{i=1, \ldots, n^{\prime}}\right. \\
& \stackrel{D}{\longrightarrow} \mathcal{N}\left(0,\left[\begin{array}{cc}
M_{\infty}\left(g^{\star}\right) & M_{\infty}\left(g^{\star}, 1\right) \\
M_{\infty}\left(g^{\star}, 1\right) & M_{\infty}(1)
\end{array}\right]\right), \text { as } n \rightarrow \infty .
\end{aligned}
$$

Now apply the Delta-method with the function $\rho(\mathbf{x}, \mathbf{y}):=\mathbf{x} / \mathbf{y}$ where $\mathbf{x}$ and $\mathbf{y}$ are real-valued vectors of size $n^{\prime}$ and the division has to be understood component-wise. The Jacobian of $\rho$ is given by the $n^{\prime} \times 2 n^{\prime}$ matrix

$$
J_{\rho}(\mathbf{x}, \mathbf{y})=\left[\operatorname{Diag}\left(y_{1}^{-1}, \ldots y_{n^{\prime}}^{-1}\right), \operatorname{Diag}\left(-x_{1} y_{1}^{-2}, \cdots-x_{n^{\prime}} y_{n^{\prime}}^{-2}\right)\right]
$$

where, for any vector $v$ of size $n^{\prime}, \operatorname{Diag}(v)$ is the diagonal matrix whose diagonal elements are the $v_{i}$, with $i=1, \ldots, n^{\prime}$. We deduce $\left(n h^{p}\right)^{1 / 2}\left(\tilde{\tau}_{1,2 \mid \mathbf{Z}=\mathbf{z}_{i}^{\prime}}-\tau_{1,2 \mid \mathbf{Z}=\mathbf{z}_{i}^{\prime}}\right)_{i=1, \ldots, n^{\prime}} \stackrel{D}{\longrightarrow} \mathcal{N}(0, \mathbb{H})$, as $n \rightarrow \infty$, setting

$$
\mathbb{H}:=J_{\rho}(\vec{\tau}, \mathbf{e})\left[\begin{array}{cc}
M_{\infty}\left(g^{\star}\right) & M_{\infty}\left(g^{\star}, 1\right) \\
M_{\infty}\left(g^{\star}, 1\right) & M_{\infty}(1)
\end{array}\right] J_{\rho}(\vec{\tau}, \mathbf{e})^{T},
$$


where $\vec{\tau}=\left(\tau_{1,2 \mid \mathbf{Z}=\mathbf{z}_{i}^{\prime}}\right)_{i=1, \ldots, n^{\prime}}$ and $\mathbf{e}$ is the vector of size $n^{\prime}$ whose all components are equal to 1 . Thus, we have $J_{\rho}(\vec{\tau}, \mathbf{e})=\left[\operatorname{I} d_{n^{\prime}},-\operatorname{Diag}(\vec{\tau})\right]$, denoting by $I d_{n^{\prime}}$ the identity matrix of size $n^{\prime}$ and by $\operatorname{Diag}(\vec{\tau})$ the diagonal matrix of size $n^{\prime}$ whose diagonal elements are the $\tau_{1,2 \mid \mathbf{z}_{i}^{\prime}}$, for $i=1, \ldots, n^{\prime}$. To be specific, we get

$$
\mathbb{H}=M_{\infty}\left(g^{\star}\right)-\operatorname{Diag}(\vec{\tau}) M_{\infty}\left(g^{\star}, 1\right)-M_{\infty}\left(g^{\star}, 1\right) \operatorname{Diag}(\vec{\tau})+\operatorname{Diag}(\vec{\tau}) M_{\infty}(1) \operatorname{Diag}(\vec{\tau}) .
$$

For $i, j$ in $\left\{1, \ldots, n^{\prime}\right\}$ and using the symmetry of the function $g^{\star}$, we obtain

$$
\begin{gathered}
{\left[M_{\infty}\left(g^{\star}\right)\right]_{i, j}=4 \int K^{2} \mathbb{1}_{\left\{\mathbf{z}_{i}^{\prime}=\mathbf{z}_{j}^{\prime}\right\}} \mathbb{E}\left[g^{\star}\left(\mathbf{X}_{1}, \mathbf{X}\right) g^{\star}\left(\mathbf{X}_{2}, \mathbf{X}\right) \mid \mathbf{Z}=\mathbf{Z}_{1}=\mathbf{Z}_{2}=\mathbf{z}_{i}^{\prime}\right] / f_{\mathbf{Z}}\left(\mathbf{z}_{i}^{\prime}\right),} \\
{\left[\operatorname{Diag}(\vec{\tau}) M_{\infty}\left(g^{\star}, 1\right)\right]_{i, j}=4 \tau_{1,2 \mid \mathbf{Z}=\mathbf{z}_{i}^{\prime}} \int K^{2} \mathbb{1}_{\left\{\mathbf{z}_{i}^{\prime}=\mathbf{z}_{j}^{\prime}\right\}} \mathbb{E}\left[g^{\star}\left(\mathbf{X}_{1}, \mathbf{X}\right) \mid \mathbf{Z}=\mathbf{Z}_{1}=\mathbf{z}_{i}^{\prime}\right] / f_{\mathbf{Z}}\left(\mathbf{z}_{i}^{\prime}\right)} \\
=4 \int K^{2} \mathbb{1}_{\left\{\mathbf{z}_{i}^{\prime}=\mathbf{z}_{j}^{\prime}\right\}} \tau_{1,2 \mid \mathbf{Z}=\mathbf{z}_{i}^{\prime}}^{2} / f_{\mathbf{Z}}\left(\mathbf{z}_{i}^{\prime}\right)=\left[M_{\infty}\left(g^{\star}, 1\right) \operatorname{Diag}(\vec{\tau})\right]_{i, j}=\left[\operatorname{Diag}(\vec{\tau}) M_{\infty}(1) \operatorname{Diag}(\vec{\tau})\right]_{i, j} .
\end{gathered}
$$

As a consequence, we obtain

$$
[\mathbb{H}]_{i, j}=\frac{4 \int K^{2} \mathbb{1}_{\left\{\mathbf{z}_{i}^{\prime}=\mathbf{z}_{j}^{\prime}\right\}}}{f_{\mathbf{Z}}\left(\mathbf{z}_{i}^{\prime}\right)}\left(\mathbb{E}\left[g^{\star}\left(\mathbf{X}_{1}, \mathbf{X}\right) g^{\star}\left(\mathbf{X}_{2}, \mathbf{X}\right) \mid \mathbf{Z}=\mathbf{Z}_{1}=\mathbf{Z}_{2}=\mathbf{z}_{i}^{\prime}\right]-\tau_{1,2 \mid \mathbf{Z}=\mathbf{z}_{i}^{\prime}}^{2}\right) .
$$

\section{A.10 Proof of Lemma 17}

Let us first evaluate the variance-covariance matrix $M_{n, n^{\prime}}:=\left[\operatorname{Cov}\left(\hat{U}_{n, i}, \hat{U}_{n, j}\right)\right]_{1 \leq i, j \leq n^{\prime}}$. Note that $\mathbb{E}\left[g_{n, i}\left(\mathbf{X}_{j}, \mathbf{Z}_{j}\right)\right]=\mathbb{E}\left[\hat{U}_{n, i}\right]=\mathbb{E}\left[U_{n, i}(g)\right]$, and that

$$
\left(\left(n h^{p}\right)^{1 / 2}\left(\hat{U}_{n, i}-\mathbb{E}\left[U_{n, i}(g)\right]\right)\right)_{i=1, \ldots, n^{\prime}}=\frac{2 h^{p / 2}}{n^{1 / 2}} \sum_{j=1}^{n}\left(g_{n, i}\left(\mathbf{X}_{j}, \mathbf{Z}_{j}\right)-\mathbb{E}\left[U_{n, i}(g)\right]\right)_{i=1, \ldots, n^{\prime}},
$$

that is a sum of independent vectors. Thus, $\operatorname{Cov}\left(\hat{U}_{n, i}, \hat{U}_{n, j}\right)=4 n^{-1} \operatorname{Cov}\left(g_{n, i}(\mathbf{X}, \mathbf{Z}), g_{n, j}(\mathbf{X}, \mathbf{Z})\right)$, for every $i, j$ in $\left\{1, \ldots, n^{\prime}\right\}$, and

$$
\begin{aligned}
\mathbb{E} & {\left[g_{n, i}(\mathbf{X}, \mathbf{Z}) g_{n, j}(\mathbf{X}, \mathbf{Z})\right] } \\
& =\int K_{h}\left(\mathbf{z}_{i}^{\prime}-\mathbf{z}\right) K_{h}\left(\mathbf{z}_{j}^{\prime}-\mathbf{z}\right) \frac{\mathbb{E}\left[g(\mathbf{X}, \mathbf{x}) K_{h}\left(\mathbf{z}_{i}^{\prime}-\mathbf{Z}\right)\right] \mathbb{E}\left[g(\mathbf{X}, \mathbf{x}) K_{h}\left(\mathbf{z}_{j}^{\prime}-\mathbf{Z}\right)\right]}{\mathbb{E}\left[K_{h}\left(\mathbf{z}_{i}^{\prime}-\mathbf{Z}\right)\right]^{2} \mathbb{E}\left[K_{h}\left(\mathbf{z}_{j}^{\prime}-\mathbf{Z}\right)\right]^{2}} f_{\mathbf{X}, \mathbf{Z}}(\mathbf{x}, \mathbf{z}) d \mathbf{x} d \mathbf{z} \\
& \sim \frac{1}{h^{p} f_{\mathbf{Z}}^{2}\left(\mathbf{z}_{i}^{\prime}\right) f_{\mathbf{Z}}^{2}\left(\mathbf{z}_{j}^{\prime}\right)} \int g\left(\mathbf{x}_{1}, \mathbf{x}\right) g\left(\mathbf{x}_{2}, \mathbf{x}\right) K_{h}\left(\mathbf{z}_{i}^{\prime}-\mathbf{z}\right) K_{h}\left(\mathbf{z}_{j}^{\prime}-\mathbf{z}\right) K_{h}\left(\mathbf{z}_{i}^{\prime}-\mathbf{w}_{1}\right) K_{h}\left(\mathbf{z}_{j}^{\prime}-\mathbf{w}_{2}\right) \\
& \times \quad f_{\mathbf{X}, \mathbf{Z}}(\mathbf{x}, \mathbf{z}) f_{\mathbf{X}, \mathbf{Z}}\left(\mathbf{x}_{1}, \mathbf{w}_{1}\right) f_{\mathbf{X}, \mathbf{Z}}\left(\mathbf{x}_{2}, \mathbf{w}_{2}\right) d \mathbf{x} d \mathbf{z} d \mathbf{x}_{1} d \mathbf{w}_{1} d \mathbf{x}_{2} d \mathbf{w}_{2} \\
& \sim \frac{1}{h^{p} f_{\mathbf{Z}}^{2}\left(\mathbf{z}_{i}^{\prime}\right) f_{\mathbf{Z}}^{2}\left(\mathbf{z}_{j}^{\prime}\right)} \int g\left(\mathbf{x}_{1}, \mathbf{x}\right) g\left(\mathbf{x}_{2}, \mathbf{x}\right) K\left(\mathbf{u}_{1}\right) K\left(\mathbf{u}_{2}\right) K(\mathbf{u}) K\left(\frac{\mathbf{z}_{j}^{\prime}-\mathbf{z}_{i}^{\prime}}{h}+\mathbf{u}\right) f_{\mathbf{X}, \mathbf{Z}}\left(\mathbf{x}, \mathbf{z}_{i}^{\prime}-h \mathbf{u}\right) \\
& \times f_{\mathbf{X}, \mathbf{Z}}\left(\mathbf{x}_{1}, \mathbf{z}_{i}^{\prime}-h \mathbf{u}_{1}\right) f_{\mathbf{X}, \mathbf{Z}}\left(\mathbf{x}_{2}, \mathbf{z}_{j}^{\prime}-h \mathbf{u}_{2}\right) d \mathbf{x} d \mathbf{u} d \mathbf{x}_{1} d \mathbf{u}_{1} d \mathbf{x}_{2} d \mathbf{u}_{2} .
\end{aligned}
$$

When $i \neq j$ and for $n$ sufficiently large, the latter term is zero because $K$ is compactly supported (Assumption 3.6(ii)). In this case, we have $\operatorname{Cov}\left(\hat{U}_{n, i}, \hat{U}_{n, j}\right)=-4 n^{-1} \mathbb{E}\left[U_{n, i}\right] \mathbb{E}\left[U_{n, j}\right] \sim-4 n^{-1} \tau_{1,2 \mid \mathbf{Z}=\mathbf{z}_{i}^{\prime}} \tau_{1,2 \mid \mathbf{Z}=\mathbf{z}_{j}^{\prime}}=$ $o\left(\left(n h^{p}\right)^{-1}\right)$. Otherwise, $i=j$ and, as $\mathbb{E}\left[g_{n, i}\left(\mathbf{X}_{1}, \mathbf{Z}_{1}\right)\right]=O(1)$, we have

$$
\begin{aligned}
\operatorname{Var}\left(\left(g_{n, i}(\mathbf{X}, \mathbf{Z})\right)^{2}\right) & \sim \frac{1}{h^{p} f_{\mathbf{Z}}^{4}\left(\mathbf{z}_{i}^{\prime}\right)} \int g\left(\mathbf{x}_{1}, \mathbf{x}\right) g\left(\mathbf{x}_{2}, \mathbf{x}\right) K\left(\mathbf{u}_{1}\right) K\left(\mathbf{u}_{2}\right) K^{2}(\mathbf{u}) f_{\mathbf{X}, \mathbf{Z}}\left(\mathbf{x}, \mathbf{z}_{i}^{\prime}-h \mathbf{u}\right) \\
& \times f_{\mathbf{X}, \mathbf{Z}}\left(\mathbf{x}_{1}, \mathbf{z}_{i}^{\prime}-h \mathbf{u}_{1}\right) f_{\mathbf{X}, \mathbf{Z}}\left(\mathbf{x}_{2}, \mathbf{z}_{i}^{\prime}-h \mathbf{u}_{2}\right) d \mathbf{x} d \mathbf{u} d \mathbf{x}_{1} d \mathbf{u}_{1} d \mathbf{x}_{2} d \mathbf{u}_{2}
\end{aligned}
$$




$$
\sim \frac{\int K^{2}}{h^{p} f_{\mathbf{Z}}\left(\mathbf{z}_{i}^{\prime}\right)} \int g\left(\mathbf{x}_{1}, \mathbf{x}\right) g\left(\mathbf{x}_{2}, \mathbf{x}\right) f_{\mathbf{X} \mid \mathbf{Z}=\mathbf{z}_{i}^{\prime}}(\mathbf{x}) f_{\mathbf{X} \mid \mathbf{Z}=\mathbf{z}_{i}^{\prime}}\left(\mathbf{x}_{1}\right) f_{\mathbf{X} \mid \mathbf{Z}=\mathbf{z}_{i}^{\prime}}\left(\mathbf{x}_{2}\right) d \mathbf{x} d \mathbf{x}_{1} d \mathbf{x}_{2}
$$

by Bochner's lemma and 3.1. We have proved that, for every $i, j \in\left\{1, \ldots, n^{\prime}\right\}$,

$$
n h^{p}\left[M_{n, n^{\prime}}\right]_{i, j} \rightarrow \frac{4 \int K^{2} \mathbb{1}_{\left\{\mathbf{z}_{i}^{\prime}=\mathbf{z}_{j}^{\prime}\right\}}}{f_{\mathbf{Z}}\left(\mathbf{z}_{i}^{\prime}\right)} \int g\left(\mathbf{x}_{1}, \mathbf{x}\right) g\left(\mathbf{x}_{2}, \mathbf{x}\right) f_{\mathbf{X} \mid \mathbf{Z}=\mathbf{z}_{i}^{\prime}}(\mathbf{x}) f_{\mathbf{X} \mid \mathbf{Z}=\mathbf{z}_{i}^{\prime}}\left(\mathbf{x}_{1}\right) f_{\mathbf{X} \mid \mathbf{Z}=\mathbf{z}_{i}^{\prime}}\left(\mathbf{x}_{2}\right) d \mathbf{x} d \mathbf{x}_{1} d \mathbf{x}_{2},
$$

as $n \rightarrow \infty$. Therefore, $n h^{p} M_{n, n^{\prime}}$ tends to $M_{\infty}$.

We now verify Lyapunov's condition with third-order moments, so that the usual multivariate central limit theorem would apply. It is then sufficient to show that

$$
\left(\frac{h^{p / 2}}{n^{1 / 2}}\right)^{3} \sum_{j=1}^{n} \mathbb{E}\left[\left|g_{n, i}\left(\mathbf{X}_{j}, \mathbf{Z}_{j}\right)-\mathbb{E}\left[U_{n, i}(g)\right]\right|^{3}\right]=o(1)
$$

For any $j=1, \ldots, n$, we have

$$
\begin{aligned}
& \mathbb{E}\left[\left|g_{n, i}\left(\mathbf{X}_{j}, \mathbf{Z}_{j}\right)-\mathbb{E}\left[U_{n, i}(g)\right]\right|^{3}\right] \\
& \sim \int\left|\frac{1}{f_{\mathbf{Z}}^{2}\left(\mathbf{z}_{i}^{\prime}\right)} \int g\left(\mathbf{x}_{1}, \mathbf{x}\right) K_{h}\left(\mathbf{z}_{i}^{\prime}-\mathbf{z}_{1}\right) K_{h}\left(\mathbf{z}_{i}^{\prime}-\mathbf{z}\right) f_{\mathbf{X}, \mathbf{Z}}\left(\mathbf{x}_{1}, \mathbf{z}_{1}\right) d \mathbf{x}_{1} d \mathbf{z}_{1}-\mathbb{E}\left[U_{n, i}(g)\right]\right|^{3} f_{\mathbf{X}, \mathbf{Z}}(\mathbf{x}, \mathbf{z}) d \mathbf{x} d \mathbf{z} .
\end{aligned}
$$

By the change of variable $\mathbf{z}_{1}=\mathbf{z}_{i}^{\prime}-h \mathbf{t}_{1}$ and $\mathbf{z}=\mathbf{z}_{i}^{\prime}-h \mathbf{t}$, we get

$$
\begin{aligned}
\mathbb{E} & {\left[\left|g_{n, i}\left(\mathbf{X}_{j}, \mathbf{Z}_{j}\right)-\mathbb{E}\left[U_{n, i}(g)\right]\right|^{3}\right] \sim h^{-2 p} \int \mid \frac{1}{f_{\mathbf{Z}}^{2}\left(\mathbf{z}_{i}^{\prime}\right)} \int g\left(\mathbf{x}_{1}, \mathbf{x}\right) K\left(\mathbf{t}_{1}\right) K(\mathbf{t}) f_{\mathbf{X}, \mathbf{Z}}\left(\mathbf{x}_{1}, \mathbf{z}_{i}^{\prime}-h \mathbf{t}_{1}\right) d \mathbf{x}_{1} d \mathbf{t}_{1} } \\
& -\left.h^{p} \mathbb{E}\left[U_{n, i}(g)\right]\right|^{3} f_{\mathbf{X}, \mathbf{Z}}\left(\mathbf{x}, \mathbf{z}_{i}^{\prime}-h \mathbf{t}\right) d \mathbf{x} d \mathbf{t}=O\left(h^{-2 p}\right),
\end{aligned}
$$

because of Bochner's lemma and Assumptions 3.1 and 3.4. Therefore, we have obtained

$$
\left(\frac{h^{p / 2}}{n^{1 / 2}}\right)^{3} \sum_{j=1}^{n} \mathbb{E}\left[\left|g_{n, i}\left(\mathbf{X}_{j}, \mathbf{Z}_{j}\right)-\mathbb{E}\left[U_{n, i}(g)\right]\right|^{3}\right]=O\left(h^{3 p / 2} n^{-3 / 2} n h^{-2 p}\right)=O\left(\left(n h^{p}\right)^{-1 / 2}\right)=o(1),
$$

applying Assumption 3.6(i). Therefore, we have checked Lyapunov's condition and the result follows.

\section{References}

[1] Acar, E. F., C. Genest, and J. Nešlehová (2012). Beyond simplified pair-copula constructions. J. Multivariate Anal. 110, 74-90.

[2] Agostinelli, C. and U. Lund (2017). circular: Circular Statistics. R package version 0.4-93. Available on CRAN.

[3] Asimit, A. V., R. Gerrard, Y. Hou, and L. Peng (2016). Tail dependence measure for examining financial extreme co-movements. J. Econometrics 194(2), 330-348.

[4] Bessec, M. and J. Fouquau (2008). The non-linear link between electricity consumption and temperature in Europe: A threshold panel approach. Energy Econ. 30(5), 2705-2721.

[5] Bosq, D. and J.-P. Lecoutre (1987). Théorie de l'Estimation Fonctionnelle. Economica, Paris.

[6] Chaieb, L. L., L.-P. Rivest, and B. Abdous (2006). Estimating survival under a dependent truncation. Biometrika 93(3), 655669.

[7] Davies, M. (1959). The relationship between weather and electricity demand. Proceedings of the IEE - Part C: Monographs 106(9), pp. 27-37.

[8] Derumigny, A. and J.-D. Fermanian (2017). About tests of the "simplifying" assumption for conditional copulas. Depend. Model. 5, 154-197.

[9] Derumigny, A. and J.-D. Fermanian (2019). A classification point-of-view about conditional Kendall's tau. Comput. Statist. Data Anal. 135, 70-94.

[10] Dony, J. and D. M. Mason (2008). Uniform in bandwidth consistency of conditional U-statistics. Bernoulli 14(4), $1108-1133$.

[11] Einmahl, U. and D. M. Mason (2005). Uniform in bandwidth consistency of kernel-type function estimators. Ann. Statist. 33(3), 1380-1403. 
[12] Fermanian, J.-D. and O. Lopez (2018). Single-index copulas. J. Multivariate Anal. 165, 27-55.

[13] Fermanian, J.-D. and M. H. Wegkamp (2012). Time-dependent copulas. J. Multivariate Anal. 110, 19-29.

[14] Filzmoser, P., H. Fritz, and K. Kalcher (2018). pcaPP: Robust PCA by Projection Pursuit. R package version 1.9-73. Available on CRAN.

[15] Gijbels, I., N. Veraverbeke, and M. Omelka (2011). Conditional copulas, association measures and their applications. Comput. Statist. Data Anal. 55(5), 1919-1932.

[16] Giné, E. and A. Guillou (2002). Rates of strong uniform consistency for multivariate kernel density estimators. Ann. Inst. Henri Poincaré Probab. Stat. 38(6), 907-921.

[17] Hobæk Haff, I., K. Aas, and A. Frigessi (2010). On the simplified pair-copula construction - Simply useful or too simplistic? J. Multivariate Anal. 101(5), 1296-1310.

[18] Hollander, M. and D. A. Wolfe (1973). Nonparametric Statistical Methods. John Wiley \& Sons, New York.

[19] Hsieh, J.-J. and W.-C. Huang (2015). Nonparametric estimation and test of conditional Kendall's tau under semi-competing risks data and truncated data. J. Appl. Stat. 42(7), 1602-1616.

[20] Joe, H. (1997). Multivariate Models and Dependence Concepts. CRC Press, Boca Raton FL.

[21] Kim, Y.-J. (2015). Estimation of conditional KendallâÁŹs tau for bivariate interval censored data. Commun. Stat. Appl. Methods 22(6), 599-604.

[22] Kruskal, W. H. (1958). Ordinal measures of association. J. Amer. Statist. Assoc. 53(284), 814-861.

[23] Lakhal, L., L.-P. Rivest, and B. Abdous (2008). Estimating survival and association in a semicompeting risks model. Biometrics 64(1), 180-188.

[24] Lehmann, E. L. (1975). Nonparametrics: Statistical Methods Based on Ranks. Holden-Day, San Francisco.

[25] Liu, A., Y. Hou, and L. Peng (2015). Interval estimation for a measure of tail dependence. Insurance Math. Econom. 64, 294-305.

[26] Major, P. (2006). An estimate on the supremum of a nice class of stochastic integrals and U-statistics. Probab. Theory Related Fields 134(3), 489-537.

[27] Manatunga, A. K. and D. Oakes (1996). A measure of association for bivariate frailty distributions. J. Multivariate Anal. 56(1), 60-74.

[28] Martin, E. C. and R. A. Betensky (2005). Testing quasi-independence of failure and truncation times via conditional Kendall's tau. J. Amer. Statist. Assoc. 100(470), 484-492.

[29] Moral-Carcedo, J. and J. Pérez-García (2019). Time of day effects of temperature and daylight on short term electricity load. Energy 174, 169-183.

[30] Nelsen, R. B. (2006). An Introduction to Copulas. Second edition. Springer, New York.

[31] Oakes, D. (1989). Bivariate survival models induced by frailties. J. Amer. Statist. Assoc. 84(406), 487-493.

[32] Pardo, A., V. Meneu, and E. Valor (2002). Temperature and seasonality influences on Spanish electricity load. Energy Econ. 24(1), 55-70.

[33] Patton, A. (2006a). Estimation of multivariate models for time series of possibly different lengths. J. Appl. Econometrics 21(2), 147-173.

[34] Patton, A. (2006b). Modelling asymmetric exchange rate dependence. Internat. Econom. Rev. 47(2), 527-556.

[35] Rinaldo, A. and L. Wasserman (2010). Generalized density clustering. Ann. Statist. 38(5), 2678-2722.

[36] Sabeti, A., M. Wei, and R. V. Craiu (2014). Additive models for conditional copulas. Stat 3(1), 300-312.

[37] Serfling, R. J. (1980). Approximation Theorems of Mathematical Statistics. John Wiley \& Sons, New York.

[38] Stute, W. (1991). Conditional U-statistics. Ann. Probab. 19(2), 812-825.

[39] Tsai, W.-Y. (1990). Testing the assumption of independence of truncation time and failure time. Biometrika 77(1), $169-177$.

[40] Veraverbeke, N., M. Omelka, and I. Gijbels (2011). Estimation of a conditional copula and association measures. Scand. J. Stat. 38(4), 766-780. 